\title{
Daily activity monitoring in patients with COPD : assessment of upper and lower extremity performance
}

Citation for published version (APA):

Annegarn, J. (2012). Daily activity monitoring in patients with COPD : assessment of upper and lower extremity performance. [Doctoral Thesis, Maastricht University]. Datawyse / Universitaire Pers Maastricht. https://doi.org/10.26481/dis.20121107ja

Document status and date:

Published: 01/01/2012

DOI:

10.26481/dis.20121107ja

Document Version:

Publisher's PDF, also known as Version of record

\section{Please check the document version of this publication:}

- A submitted manuscript is the version of the article upon submission and before peer-review. There can be important differences between the submitted version and the official published version of record.

People interested in the research are advised to contact the author for the final version of the publication, or visit the DOI to the publisher's website.

- The final author version and the galley proof are versions of the publication after peer review.

- The final published version features the final layout of the paper including the volume, issue and page numbers.

Link to publication

\footnotetext{
General rights rights.

- You may freely distribute the URL identifying the publication in the public portal. please follow below link for the End User Agreement:

www.umlib.nl/taverne-license

Take down policy

If you believe that this document breaches copyright please contact us at:

repository@maastrichtuniversity.nl

providing details and we will investigate your claim.
}

Copyright and moral rights for the publications made accessible in the public portal are retained by the authors and/or other copyright owners and it is a condition of accessing publications that users recognise and abide by the legal requirements associated with these

- Users may download and print one copy of any publication from the public portal for the purpose of private study or research.

- You may not further distribute the material or use it for any profit-making activity or commercial gain

If the publication is distributed under the terms of Article $25 \mathrm{fa}$ of the Dutch Copyright Act, indicated by the "Taverne" license above, 
Daily activity monitoring in patients with COPD Assessment of upper and lower extremity performance 
ISBN: 9789461591753

Ccopyright J. Annegarn, Maastricht 2012

Printing: Datawyse | Universitaire Pers Maastricht

Cover design: Ilaria Chiaratti (IDA LifeStyle)

Cover drawings: Angelique Hendriks

Printing of this thesis was financially supported by the Faculty of Health, Medicine and Life Sciences, Maastricht University; $\mathrm{CIRO}+$, centre of expertise for chronic organ failure; Netherlands Asthma Foundation; Maastricht Instruments. 


\title{
Daily activity monitoring in patients with COPD
} Assessment of upper and lower extremity performance

\author{
PROEFSCHRIFT \\ ter verkrijging van de graad van doctor \\ aan de Universiteit Maastricht \\ op gezag van de Rector Magnificus \\ Prof. dr. L.L.G. Soete \\ volgens het besluit van het College van Decanen, \\ in het openbaar te verdedigen \\ op woensdag 7 november 2012 om 10.00 uur \\ door
}

Janneke Annegarn

Geboren te Eindhoven op 16 september 1984

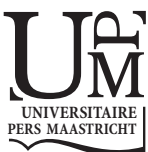




\section{Promotores}

Prof. dr. E.F.M. Wouters

Prof. dr. A.M.W.J. Schols

\section{Copromotores}

Dr. K. Meijer

Dr. M.A. Spruit

\section{Beoordelingscommissie}

Prof. dr. L.W. van Rhijn (voorzitter)

Prof. dr. H.P. Brunner-La Rocca

Prof. dr. M.K.C. Hesselink

Prof. dr. S.J. Singh

Prof. dr. W. Zijlstra

The research presented in this thesis was carried out at the Department of Human Movement Sciences, Maastricht University and $\mathrm{CIRO}+$, centre of expertise for chronic organ failure. 


\section{Contents}

1 General introduction $\quad 7$

$2 \quad$ Problematic activities of daily life are weakly associated with clinical characteristics in COPD

Objective physical activity assessment in patients

3 with chronic organ failure: a validation study of a new single-unit activity monitor

Differences in walking pattern during 6-min walk

4 test between patients with COPD and healthy subjects

5 Assessment of intensity and elevation of arm movements during activities of daily life

Characteristics of daily arm activities in patients with chronic obstructive pulmonary disease

7 General Discussion

Summary

Samenvatting

Appendix Dankwoord

Curriculum Vitae

List of publications

List of abbreviations 

Chapter 1

General introduction 


\section{Introduction}

With advancing age, structural and functional deterioration occurs in most physiological systems, even in the absence of discernible disease [1]. These age-related physiological changes affect a broad range of tissues, organ systems, and functions, which, cumulatively impact activities of daily living (ADL) and the preservation of physical independence in older adults [2].

Older populations exhibit the highest prevalence of musculoskeletal conditions such as osteoporosis, arthritis, and sarcopenia [3,4]. Moreover, the relative risk of developing chronic diseases including Chronic Obstructive Pulmonary Disease (COPD), Chronic Heart Failure (CHF), type 2 diabetes and certain cancers increases with advancing age. Physical inactivity is an important lifestyle factor that adversely modulates chronic disease risk and progression [2,5]. Otherwise regular physical activity increases average life expectancy and health status, even at high age, through the mitigation of age- and disease related biological changes, their associated effects on health and well-being, and through the preservation of functional capacity [2]. Physical activity is defined as any bodily movement produced by skeletal muscles that requires energy expenditure [6]. The physical activity recommendation for older is to accumulate moderate intensity activities at least 30 or up to 60 (for greater benefit) minutes per day in bouts of at least 10 minutes [2]. On a scale of 0 to 10 for level of physical exertion, moderate-intensity has a level of 5 to 6 . More recommendations are described in detail elsewhere [2]. Beneficial effects of physical activity are particularly evident on skeletal muscle and cardiovascular function. These effects even occur despite progressive and irreversible deterioration of the primary organ impairment (for example cardiac and pulmonary function) that is only a poor predictor of exercise capacity in advanced chronic diseases $[7,8]$. This thesis will focus on the assessment of physical functioning and physical activity in chronic progressively disabling diseases (like COPD and CHF) with a particular focus on activities of daily living as they are important for health related quality of life but also are difficult to objectively quantify. The emphasis of this thesis lies on monitoring daily activities in COPD patients.

\section{COPD prevalence and management}

COPD is defined as: 'a common preventable and treatable disease, characterized by persistent airflow limitation that is usually progressive and associated with an enhanced chronic inflammatory response in the airways and the lung to noxious parti- 
cles or gases. Exacerbations and comorbidities contribute to the overall severity in individual patients' [9]. Global Initiative for Chronic Obstructive Lung Disease (GOLD) graded severity of COPD according to the degree of airflow limitation into mild, moderate, severe and very severe (table 1.1).

Table 1.1 GOLD classification of COPD severity based on post-bronchodilator $\mathrm{FEV}_{1}$

\begin{tabular}{lll}
\hline Stage & FEV1/FVC & Post-bronchodilator FEV1 predicted \\
\hline I: Mild & $<0.70$ & FEV1 $\geq 80 \%$ \\
II: Moderate & $<0.70$ & $50 \% \leq \mathrm{FEV} 1<80 \%$ \\
III: Severe & $<0.70$ & $30 \% \leq \mathrm{FEV} 1<50 \%$ \\
IV: Very Severe & $<0.70$ & FEV1 $<30 \%$ or \\
& & FEV1 $<50 \%$ plus chronic respiratory failure
\end{tabular}

Abbreviations: $\mathrm{FEV}_{1}$ : forced expiratory volume in one second; FVC: forced vital capacity; Respiratory failure: arterial partial pressure of oxygen $\left(\mathrm{PaO}_{2}\right)$ less than $8.0 \mathrm{kPa}(60 \mathrm{~mm} \mathrm{Hg})$ with or without arterial partial pressure of $\mathrm{CO}_{2}\left(\mathrm{PaCO}_{2}\right)$ greater than $6.7 \mathrm{kPa}(50 \mathrm{~mm} \mathrm{Hg})$ while breathing air at sea level.

COPD also has important consequences at population level, as reflected by its worldwide increasing prevalence ranging from 6-26\% [10,11,12,13,14] and associated high healthcare costs $[15,16]$. COPD is expected to be the fourth leading cause of death by 2030 worldwide [17]. In the Netherlands, overall incidence of COPD in a population-based elderly cohort was estimated at 9.2/1000 person-years [18]. More recently it was estimated that $24 \%$ of the population in the area of Maastricht aged $\geq 40$ years has airflow limitation [19]. Given the demographic shift of an aging population and current smoking behavior, future scenarios expect the prevalence of COPD in the Dutch population of all ages to increase to 33 per 1000 male inhabitants and 27 per 1000 female inhabitants [20].

Though COPD is defined based on airflow limitation, the degree of airflow limitation has only a weak relation with the number of problems with the performance of daily activities a patient encounters [21,22]. Therefore, optimal management of COPD does not only focus on airflow limitation, but also on the systemic manifestations like physical functioning (e.g. physical inactivity and muscle weakness as described below), body composition and psychosocial functioning [23] in order to improve patients' participation in everyday activities, quality of life and the probability of survival [21]. Management of COPD includes reduction of risk factors, like smoking cessation; pharmacological treatment, such as bronchodilators and gluco- 
corticosteroids; and non-pharmacological treatment, such as pulmonary rehabilitation, long-term oxygen therapy and surgical treatment [9].

Dyspnea and activity limitation are the primary symptoms of COPD which worsens over time [24]. As a result, many COPD patients experience problems with ADL that cause loss of independence and social isolation and thus greatly reduce their quality of life $[25,26,27]$. Pulmonary rehabilitation aims to reduce symptoms, improve quality of life, and increase physical and emotional participation in everyday activities $[28,29]$. To accomplish these goals, pulmonary rehabilitation covers a range of nonpulmonary problems including de-conditioning, relative social isolation, altered mood states, muscle weakness and weight loss $[9,30]$. Functional status is a patient oriented meaningful health outcome, which concerns individual daily functioning. Because functional status is the most important factor affecting health related quality of life and health care utilization in old age [31] valid outcome measures of physical activity and/or physical functioning are of key importance to evaluate the success of treatment programs for COPD patients.

\section{Physical inactivity and muscle weakness in COPD}

COPD patients often have a low level of physical activity [28,29]. Pitta et al., found that COPD patients walk half the time of healthy elderly and with a lower movement intensity [32]. Sedentary or inactive behavior (taking less then 5,000 steps/day [33]) and low active behavior (taking 5000-7499 steps/day [33]) have been related to multiple important clinical outcomes, like frequent exacerbations (two or more in the preceding year), dyspnea, fatigue, and an impaired health status and mortality [34]. Moreover, sedentary behavior is believed to be crucial to the development of skeletal muscle weakness in COPD patients $[35,36,37]$. Different studies show that COPD patients suffer from reduced quadriceps muscle strength defined as the capacity of the muscle to develop maximal force $[38,39,40]$ and reduced quadriceps endurance capacity of the muscle to sustain mechanical output during loaded contraction(s) $[36,41,42,43]$. Quadriceps muscle function is moreover significantly correlated with daily walking time [32]. Muscle weakness is of much clinical interest, because it contributes to exercise limitation $[44,45]$ increased use of health care services [46] and mortality [47].

Hartman et al., (2010) has created a schematic overview showing the possible roles and consequences of physical inactivity for important COPD outcomes (figure 1.1) [35]. In this framework physical inactivity appears to be an important modifiable

risk factor. COPD patients with low levels of physical activity have doubled the odds of an increase in disability [48], have increased risk for hospital admission and have 
significantly enhanced mortality $[49,50]$. Moreover, inactivity is a mediator in the dyspnea-inactivity-deconditioning spiral. In this spiral dyspnea, fatigue, and other symptoms produce functional impairment and disability in COPD. Patients with COPD experience a reduction in muscle function and the ability to perform activities of daily life during the course of their disease [28]. These impairments are of concern because they limit the patients' independence [51,52].

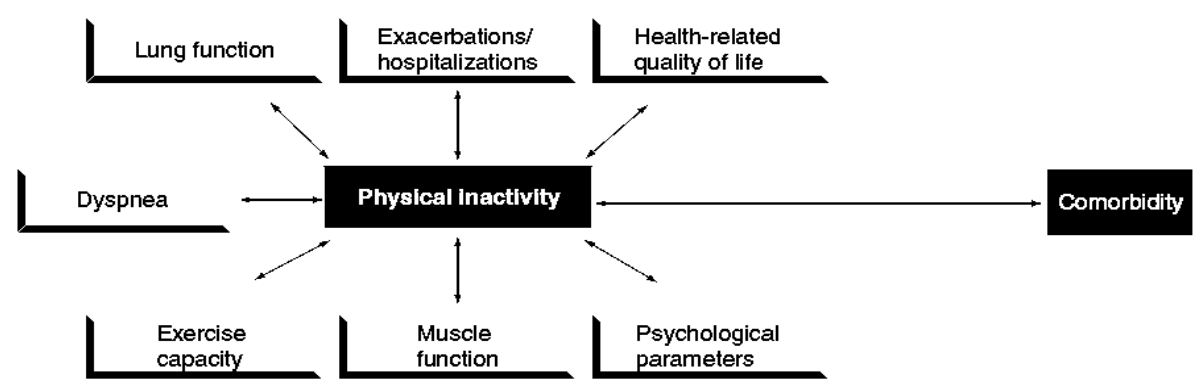

1.1: schematıc overview of the role of physıcal ınactıvity in chronıc obstructive puimonary disease. Ine possible role and consequences of physical inactivity for important COPD outcomes is shown. These relationships are embedded in COPD-related background factors. Next to COPD-related factors, the presence of disease other than COPD (called comorbidity) may influence physical activity. Adapted from Hartman et al., (2010) [35].

\section{Problematic activities in daily life; a patient's perspective}

For the assessment and evaluation of treatment programs that aim to improve daily activity performance in COPD patients, accurate measurements of the patient's ability to perform activities in daily life are essential. COPD can lead to reductions across a wide array of performance areas (e.g. to perform personal care tasks, household tasks, ambulation like walking and cycling, recreation and social activities). Some areas may be more salient to a patient than others. Patient reported questionnaires show good validity for measuring general problematic daily life activities, but poorly identify patients' individual needs [53]. An overview of the distribution of occurrence of problematic activities of daily life using semi-structured interviews in a large cohort of COPD patients is currently lacking. Insights in performance and satisfaction of problematic activities of daily life may allow clinicians to better monitor patients with COPD. 


\section{Objective activity assessment}

Though interviews or questionnaires are central in the assessment of the patients' view on their problematic activities, they show limited validity and reliability to objectively assess information on performance, duration and intensity of various daily activities [54]. Alternatively, unobtrusive wearable devices gain popularity as method for physical activity assessment $[55,56,57,58,59,60,61,62,63]$. Accelerometer-based activity monitors are most widely used wearable systems in COPD patients as they are able to record unobtrusively under free-living conditions for prolonged periods of time to account for day-to-day variability [64]. Accelerometers respond to the dynamic forces during movement and allow the assessment of movement intensity, frequency and duration. Moreover accelerometers measure gravitational acceleration which enables the detection of specific postures (e.g. standing, sitting and lying). Physical activity recognition techniques can be subsequently used to transform raw accelerometer signals into information on functional performance of the upper and lower limbs. These techniques allow detailed analyses of the impact of COPD on the functional performance of individual patients.

Research on daily activity assessment in COPD patients has been focused on the lower limbs, also referred to as whole-body activities as the lower limbs are responsible for ambulation [32]. Different types of instruments have been used to measure physical activity in older persons $[65,66]$ and specifically in COPD patients [58]. These instruments vary in reported outcomes and by the location, type and number of accelerometers used. Most systems report physical activity expressed as time spent on movements, intensity of movement periods or estimates of energy expenditure as primary outcomes $[67,68,69,70]$. Physiologically, distinct effects are observed between prolonged sedentary (=sitting) time and too little physical activity [71]. Rapidly-strengthening evidence indicate that too much sitting is a real and substantial risk to health [72]. Distinction between sitting and standing provides important additional information on sedentary behavior, which can be particularly relevant to the COPD population who are in general characterized by an inactive lifestyle [32]. For example, compared to healthy subjects COPD patients show on average an increased sitting and lying time, whereas standing time is decreased [32]. Exchanging daily standing time for sitting or lying time might therefore be an important sign of decline in the individual patient which would have been missed using instruments unable to distinguish between postures. Available activity monitors validated in the COPD population show important limitations, such as being obtrusive, consisting of multiple units [54], not validated during 'free living' conditions [54], or unable to differentiate accurately between postures [73]. Therefore there is a need for an accurate unobtrusive single-unit monitor for measuring sedentary behavior validated in the COPD population under 'free living' conditions. 
Patients who experience problems with daily life activities may not only lower standing time, activity time or activity intensity, but also adapt the performance of problematic activities of daily life. Walking is frequently reported to be problematic in daily life by COPD patients $[26,74]$. Perhaps the most robust use for accelerometer-based walking analysis is the determination of spatio-temporal parameters from foot contact events [75]. For example, from the impact of heel strike, stride (=two steps) duration can been calculated [76]. Adaptations in spatio-temporal parameters have been associated with physical fatigue $[77,78]$ balance dysfunction $[79,80,81]$ and heightened fall risk (e.g., see figure 1.2$)[79,80]$. This is of particular interest in COPD patients who are prone to osteoporosis [82], have a disturbed balance [83] and a high susceptibility to falls [84]. To date, detailed analyses of walking patterns using an accelerometer have not yet been performed in patients with COPD.
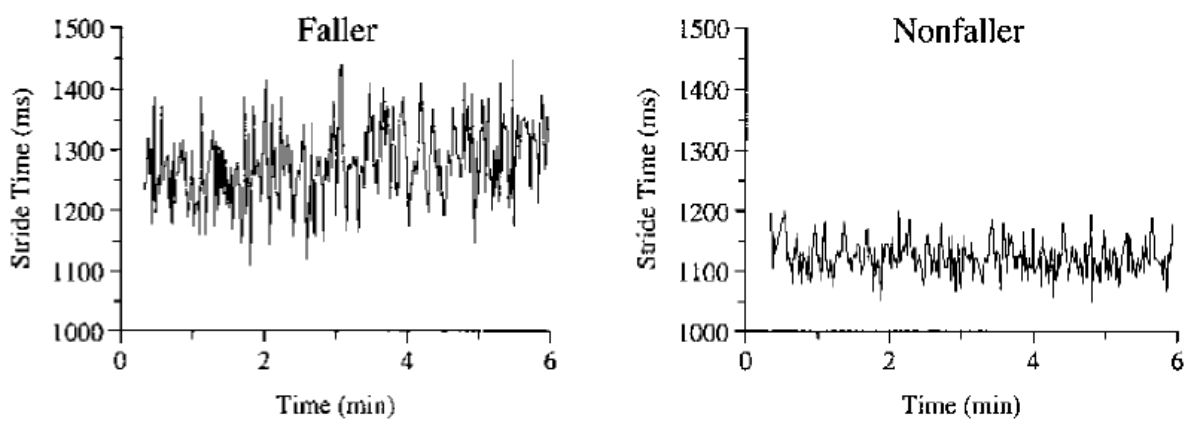

Figure 1.2: Stride-to-stride fluctuations in the stride time, as measured at baseline, were much larger in the participant who experienced a fall during the 12 month follow-up period, as compared to the nonfallers. Group results were similar. Adapted from Hausdorff et al., (2001) [85].

Research on muscle activity during ADLs is limited. Surface Electromyography (sEMG) can provide useful indices reflecting motor unit recruitment and represent an attractive means to assess muscle effort during the performance of ADLs. Marquis et al., used electromyography (EMG) to assess muscle activity profiles during walking and found similar activation profile of the lower limb muscles between COPD patients and healthy controls, despite a much lower walking speed in the former group [86]. It is conceivable that the observed EMG changes would have been more profound in patients with COPD when they had walked at the same speed as healthy controls. For this reason authors suggested that COPD patients are more vulnerable to muscle fatigue [86]. Lower limb muscle function is important to characterize in COPD patients, as it is largely responsible for limiting common daily activities such as walking [32]. 
In COPD patients daily upper limb activities, such as self-grooming, cleaning the window, and sweeping the floor can also be impaired and symptom-inducing [87]. These impairments are of concern because they limit the patients' independence during day-to-day life [51,52]. Despite the availability of several studies on daily activity assessment of the whole-body or lower limbs, thus far, no study evaluated specifically upper limb daily use in COPD patients. COPD patients report a marked increase in the sensation of dyspnea and fatigue with routine tasks that require upper limb use, especially activities necessitating unsupported arm elevation $[88,89,90]$. Consequently, to reduce the burden of fatigue and dyspnea, patients may reduce upper limb activities in daily life. Nevertheless, upper limb activities are still required for maintenance of self-care and independent living even if activities require more effort $[91,92,93,94]$. The impact of COPD on daily upper limb use is currently unknown.

Compared to the clear categories defined for lower limb activities and postures (e.g. standing, sitting, ambulation), upper limb activities are much more diverse (cleaning, eating, dressing, etc.). Therefore multiple sensor systems have been developed to assess three-dimensional kinematics of the upper limbs $[95,96,97,98,99,100]$. While these more sophisticated sensors hold promise, they also have limitations, such as being large, requiring cables and data loggers worn on a belt, moreover they can measure for only short periods of time and are either very expensive or not commercially available. In addition, generally complex methods are necessary to translate raw data of multiple sensor systems into detailed information on daily upper limb use [101]. To evaluate relevant information on daily upper extremity use in COPD patients, the necessity of multiple sensors is questionable. Accelerometers, small single-unit sensor systems, are less obtrusive and can measure for extensive periods of time. Because less precision can be achieved (e.g. accelerometers do not provide information on movements specifically within the horizontal plane) categories which define different types of movement need to be selected. Movement intensity and elevation of the upper extremities are especially important to assess in COPD patients, because they have been shown to impair ventilatory mechanisms and increase dyspnea and fatigue [89,90,102,103]. In addition arm intensity and arm elevation provide important information on functional use $[96,100,104,105]$. There is a need to evaluate the ability of accelerometers to assess levels of movement intensity and elevation of the upper extremity.

Skeletal muscle weakness in COPD patients appears to be particularly localized to locomotor muscles, such as the quadriceps. Preservation of upper body muscles function has been found in terms of strength (e.g. neck flexion and adductor pollicis muscles $[39,40,106])$ and endurance (e.g. triceps, deltoid and biceps muscles $[91,92,93])$ These findings are clearly different from results on the quadriceps mus- 
cle and may be due to the maintenance of ADLs that involve the upper body muscles or to the use of some of those muscles during the work of breathing. However, no study has yet investigated upper body muscle activity profiles in relation to the performances of daily activities. It has been found that COPD experience a relatively high metabolic load and dyspnea during the performance of self-paced domestic arm ADLs compared to healthy controls subjects [87]. Consequently, COPD patients may use their muscles involving breathing to a higher extend during the performance of daily activities compared to healthy subject. Alternatively, COPD patient may adapt their daily activity performance of the upper body to maintain similar muscle activity profiles as healthy subjects. Upper body activities are required for maintenance of self-care and independent living. Better insight in the performance of daily activities in relation to muscle activity profiles of the upper body is therefore very important and may help moving treatment forward to improve patients' independence in daily life.

\section{Aim of the thesis}

Patients with COPD experience a progressive reduction in the ability to perform daily life activities during the course of their disease. Inactivity plays an important role in the progression of COPD. Wearable devices able to monitor the activities in daily life are about to become central in preventive medicine and disease management. A more in depth knowledge concerning the activities that appear important to monitor is therefore critical. For that reason, this thesis encompasses two objectives. The first objective is to identify the most prevalent problematic activities of daily life in patients with COPD and explore their association with clinical determinants. The second objective is to evaluate the time spend and the performance of daily lower and upper body activities using tri-axial accelerometers of COPD patients and healthy subjects. Furthermore hardware and software needed to enable measurements of daily physical activity of lower and upper extremities were developed and validated as part of this thesis.

\section{Thesis outline}

This thesis describes a series of studies in which daily life activities are evaluated in COPD patients. The project started with identifying to the most prevalent problematic daily activities in patients with COPD using semi-structured interviews. Moreover, the association between clinical determinants and problematic daily activities were investigated (Chapter 2, Problematic activities of daily life are weakly associated with clinical characteristics in COPD). With the insight in the problematic 
daily activities we developed and evaluated a new tri-axial accelerometer to objectively assess leg activities of patients with COPD and CHF with a wide range of functional exercise capacity during 'free living' conditions (Chapter 3, Objective physical activity assessment in patients with chronic organ failure: a validation study of a new single-unit activity monitor). Moreover, we decided to study the walking pattern in COPD patients in more detail during the six-minute walk test because the sixminute walk distance is commonly used to assess functional performance and has prognostic value (Chapter 4, Differences in walking pattern during 6-min walk test between COPD and healthy subjects). Because COPD patients experience a relatively high metabolic load and dyspnea during the performance of daily arm activities compared to healthy subjects, we furthermore used triaxial accelerometers to objectively assess arm activities (Chapter 5, assessment of intensity and elevation of arm movements during activities of daily life). Finally, based on the methods to assess daily leg and arm activities in daily life, we aimed at quantifying and comparing arm and leg activities during daily life of COPD patients and healthy subjects (Chapter 6, Characteristics of daily arm activities in patients with chronic obstructive pulmonary disease). This chapter moreover describes and evaluates ADL performances and associated relative effort of upper body muscles of COPD patients and healthy subjects in a controlled setting. In the last chapter (Chapter 7, general discussion) we finally discuss the outcome of the studies described in chapters 2-6, technological developments, methodological considerations and recommendations for future research. 


\section{References}

1. Masoro E (1995) Handbook of Physiology Section 11. In: Masoro E, editor. New York (NY): Oxford University Press. pp. 3-21.

2. Chodzko-Zajko WJ, Proctor DN, Fiatarone Singh MA, Minson CT, Nigg CR, et al. (2009) American College of Sports Medicine Position Stand. Exercise and physical activity for older adults. Med Sci Sports Exerc 41: 1510-1530.

3. Ostchega $Y$, Harris TB, Hirsch R, Parsons VL, Kington R (2000) The prevalence of functional limitations and disability in older persons in the US: data from the National Health and Nutrition Examination Survey III. J Am Geriatr Soc 48: 1132-1135.

4. Paterson D (2002) Physical activity, fitness, and gender in relation to morbidity, survival, quality of life, and independence in older age. Boca Raton (FL): CRC Press.

5. Matheson GO, KlugI M, Dvorak J, Engebretsen L, Meeuwisse WH, et al. (2011) Responsibility of sport and exercise medicine in preventing and managing chronic disease: applying our knowledge and skill is overdue. Br J Sports Med 45: 1272-1282.

6. Caspersen CJ, Christenson GM, Pollard RA (1986) Status of the 1990 physical fitness and exercise objectives--evidence from NHIS 1985. Public Health Rep 101: 587-592.

7. Gosker HR, Lencer NH, Franssen FM, van der Vusse GJ, Wouters EF, et al. (2003) Striking similarities in systemic factors contributing to decreased exercise capacity in patients with severe chronic heart failure or COPD. Chest 123: 1416-1424.

8. Spruit MA, Watkins ML, Edwards LD, Vestbo J, Calverley PM, et al. (2010) Determinants of poor 6min walking distance in patients with COPD: the ECLIPSE cohort. Respir Med 104: 849-857.

9. Rabe KF, Hurd S, Anzueto A, Barnes PJ, Buist SA, et al. (2011) Global strategy for the diagnosis, management, and prevention of chronic obstructive pulmonary disease: GOLD executive summary (revised 2011). wwwgoldcopdorg.

10. Halbert RJ, Natoli JL, Gano A, Badamgarav E, Buist AS, et al. (2006) Global burden of COPD: systematic review and meta-analysis. Eur Respir J 28: 523-532.

11. Mannino DM, Buist AS (2007) Global burden of COPD: risk factors, prevalence, and future trends. Lancet 370: 765-773.

12. Buist AS, McBurnie MA, Vollmer WM, Gillespie S, Burney $P$, et al. (2007) International variation in the prevalence of COPD (the BOLD Study): a population-based prevalence study. Lancet 370: 741-750.

13. Mannino DM, Homa DM, Akinbami LJ, Ford ES, Redd SC (2002) Chronic obstructive pulmonary disease surveillance--United States, 1971-2000. Respir Care 47: 1184-1199.

14. Menezes AM, Perez-Padilla R, Jardim JR, Muino A, Lopez MV, et al. (2005) Chronic obstructive pulmonary disease in five Latin American cities (the PLATINO study): a prevalence study. Lancet 366: 1875-1881.

15. Pauwels RA, Buist AS, Calverley PM, Jenkins CR, Hurd SS (2001) Global strategy for the diagnosis, management, and prevention of chronic obstructive pulmonary disease. NHLBI/WHO Global Initiative for Chronic Obstructive Lung Disease (GOLD) Workshop summary. Am J Respir Crit Care Med 163: 1256-1276.

16. Chapman KR, Mannino DM, Soriano JB, Vermeire PA, Buist AS, et al. (2006) Epidemiology and costs of chronic obstructive pulmonary disease. Eur Respir J 27: 188-207.

17. Mathers CD, Loncar D (2006) Projections of global mortality and burden of disease from 2002 to 2030. PLoS Med 3: e442.

18. van Durme YM, Verhamme KM, Stijnen T, van Rooij FJ, Van Pottelberge GR, et al. (2009) Prevalence, incidence, and lifetime risk for the development of COPD in the elderly: the Rotterdam study. Chest 135: 368-377. 
19. Vanfleteren LE, Franssen FM, Wesseling G, Wouters EF (2012) The prevalence of chronic obstructive pulmonary disease in Maastricht, the Netherlands. Respir Med 106: 871-874.

20. Hoogendoorn M, Rutten-van Molken MP, Hoogenveen RT, van Genugten ML, Buist AS, et al. (2005) A dynamic population model of disease progression in COPD. Eur Respir J 26: 223-233.

21. Agusti A, Calverley PM, Celli B, Coxson HO, Edwards LD, et al. (2010) Characterisation of COPD heterogeneity in the ECLIPSE cohort. Respir Res 11: 122.

22. Jones PW (2009) Health status and the spiral of decline. Copd 6: 59-63.

23. Spruit MA, Vanderhoven-Augustin I, Janssen PP, Wouters EF (2008) Integration of pulmonary rehabilitation in COPD. Lancet 371: 12-13.

24. O'Donnell DE, Laveneziana P (2007) Dyspnea and activity limitation in COPD: mechanical factors. Copd 4: 225-236.

25. Griffith L, Raina P, Wu H, Zhu B, Stathokostas L (2010) Population attributable risk for functional disability associated with chronic conditions in Canadian older adults. Age Ageing 39: 738-745.

26. Williams V, Bruton A, Ellis-Hill C, McPherson K (2007) What really matters to patients living with chronic obstructive pulmonary disease? An exploratory study. Chron Respir Dis 4: 77-85.

27. Jeon YH, Kraus SG, Jowsey T, Glasgow NJ (2010) The experience of living with chronic heart failure: a narrative review of qualitative studies. BMC Health Serv Res 10: 77.

28. Nici L, Donner C, Wouters E, Zuwallack R, Ambrosino N, et al. (2006) American Thoracic Society/European Respiratory Society statement on pulmonary rehabilitation. Am J Respir Crit Care Med 173: $1390-1413$.

29. Ries AL, Bauldoff GS, Carlin BW, Casaburi R, Emery CF, et al. (2007) Pulmonary Rehabilitation: Joint ACCP/AACVPR Evidence-Based Clinical Practice Guidelines. Chest 131: 4S-42S.

30. Lacasse Y, Brosseau L, Milne S, Martin S, Wong E, et al. (2002) Pulmonary rehabilitation for chronic obstructive pulmonary disease. Cochrane Database Syst Rev: CD003793.

31. Ferrucci L, Baldasseroni S, Bandinelli S, de Alfieri W, Cartei A, et al. (2000) Disease severity and health-related quality of life across different chronic conditions. J Am Geriatr Soc 48: 1490-1495.

32. Pitta F, Troosters T, Spruit MA, Probst VS, Decramer M, et al. (2005) Characteristics of physical activities in daily life in chronic obstructive pulmonary disease. Am J Respir Crit Care Med 171: 972 977.

33. Tudor-Locke C, Craig CL, Aoyagi Y, Bell RC, Croteau KA, et al. How many steps/day are enough? For older adults and special populations. Int J Behav Nutr Phys Act 8: 80.

34. Waschki B, Spruit MA, Watz H, Albert PS, Shrikrishna D, et al. (2011) Physical activity monitoring in COPD: Compliance and associations with clinical characteristics in a multicenter study. Respir Med 106: $522-530$.

35. Hartman JE, Boezen HM, de Greef MH, Bossenbroek L, ten Hacken NH (2011) Consequences of physical inactivity in chronic obstructive pulmonary disease. Expert Rev Respir Med 4: 735-745.

36. Serres I, Gautier V, Varray A, Prefaut C (1998) Impaired skeletal muscle endurance related to physical inactivity and altered lung function in COPD patients. Chest 113: 900-905.

37. Man WD, Kemp P, Moxham J, Polkey MI (2009) Exercise and muscle dysfunction in COPD: implications for pulmonary rehabilitation. Clin Sci (Lond) 117: 281-291.

38. Bernard S, LeBlanc P, Whittom F, Carrier G, Jobin J, et al. (1998) Peripheral muscle weakness in patients with chronic obstructive pulmonary disease. Am J Respir Crit Care Med 158: 629-634.

39. Man WD, Soliman MG, Nikoletou D, Harris ML, Rafferty GF, et al. (2003) Non-volitional assessment of skeletal muscle strength in patients with chronic obstructive pulmonary disease. Thorax 58: 665669.

40. Man WD, Hopkinson NS, Harraf F, Nikoletou D, Polkey MI, et al. (2005) Abdominal muscle and quadriceps strength in chronic obstructive pulmonary disease. Thorax 60: 718-722.

41. Van't Hul A, Harlaar J, Gosselink R, Hollander P, Postmus P, et al. (2004) Quadriceps muscle endurance in patients with chronic obstructive pulmonary disease. Muscle Nerve 29: 267-274.

42. Allaire J, Maltais F, Doyon JF, Noel M, LeBlanc P, et al. (2004) Peripheral muscle endurance and the oxidative profile of the quadriceps in patients with COPD. Thorax 59: 673-678. 
43. Swallow EB, Gosker HR, Ward KA, Moore AJ, Dayer MJ, et al. (2007) A novel technique for nonvolitional assessment of quadriceps muscle endurance in humans. J Appl Physiol 103: 739-746.

44. Gosselink R, Troosters T, Decramer M (1996) Peripheral muscle weakness contributes to exercise limitation in COPD. Am J Respir Crit Care Med 153: 976-980.

45. Hamilton AL, Killian KJ, Summers E, Jones NL (1995) Muscle strength, symptom intensity, and exercise capacity in patients with cardiorespiratory disorders. Am J Respir Crit Care Med 152: 2021-2031.

46. Decramer M, Gosselink R, Troosters T, Verschueren M, Evers G (1997) Muscle weakness is related to utilization of health care resources in COPD patients. Eur Respir J 10: 417-423.

47. Swallow EB, Reyes D, Hopkinson NS, Man WD, Porcher R, et al. (2007) Quadriceps strength predicts mortality in patients with moderate to severe chronic obstructive pulmonary disease. Thorax 62 : 115-120.

48. Katz P, Chen H, Omachi TA, Gregorich SE, Julian L, et al. (2011) The Role of Physical Inactivity in Increasing Disability Among Older Adults With Obstructive Airway Disease. J Cardiopulm Rehabil Prev 31: 193-197.

49. Garcia-Aymerich J, Lange P, Benet M, Schnohr P, Anto JM (2006) Regular physical activity reduces hospital admission and mortality in chronic obstructive pulmonary disease: a population based cohort study. Thorax 61: 772-778.

50. Waschki B, Kirsten A, Holz O, Muller KC, Meyer T, et al. (2011) Physical Activity Is the Strongest Predictor of All-Cause Mortality in Patients With COPD: A Prospective Cohort Study. Chest 140: 331 342.

51. Guccione AA, Felson DT, Anderson JJ, Anthony JM, Zhang Y, et al. (1994) The effects of specific medical conditions on the functional limitations of elders in the Framingham Study. Am J Public Health 84: 351-358.

52. Rodriguez Gonzalez-Moro JM, de Lucas Ramos P, Izquierdo Alonso JL, Lopez-Muniz Ballesteros B, Anton Diaz E, et al. (2009) Impact of COPD severity on physical disability and daily living activities: EDIP-EPOC I and EDIP-EPOC II studies. Int J Clin Pract 63: 742-750.

53. Stull DE, Leidy NK, Jones PW, Stahl E (2007) Measuring functional performance in patients with COPD: a discussion of patient-reported outcome measures. Curr Med Res Opin 23: 2655-2665.

54. Pitta F, Troosters T, Spruit MA, Decramer M, Gosselink R (2005) Activity monitoring for assessment of physical activities in daily life in patients with chronic obstructive pulmonary disease. Arch Phys Med Rehabil 86: 1979-1985.

55. Troosters T, Sciurba F, Battaglia S, Langer D, Valluri SR, et al. (2010) Physical inactivity in patients with COPD, a controlled multi-center pilot-study. Respir Med 104: 1005-1011.

56. Furlanetto KC, Bisca GW, Oldemberg N, Sant'anna TJ, Morakami FK, et al. (2010) Step counting and energy expenditure estimation in patients with chronic obstructive pulmonary disease and healthy elderly: accuracy of 2 motion sensors. Arch Phys Med Rehabil 91: 261-267.

57. van den Berg-Emons RJ, Bussmann JB, Balk AH, Stam HJ (2005) Factors associated with the level of movement-related everyday activity and quality of life in people with chronic heart failure. Phys Ther 85: 1340-1348.

58. Pitta F, Troosters T, Probst VS, Spruit MA, Decramer M, et al. (2006) Quantifying physical activity in daily life with questionnaires and motion sensors in COPD. Eur Respir J 27: 1040-1055.

59. Jehn M, Schmidt-Trucksass A, Schuster T, Weis M, Hanssen H, et al. (2009) Daily walking performance as an independent predictor of advanced heart failure: Prediction of exercise capacity in chronic heart failure. Am Heart J 157: 292-298.

60. Grant PM, Ryan CG, Tigbe WW, Granat MH (2006) The validation of a novel activity monitor in the measurement of posture and motion during everyday activities. Br J Sports Med 40: 992-997.

61. Hecht A, Ma S, Porszasz J, Casaburi R (2009) Methodology for using long-term accelerometry monitoring to describe daily activity patterns in COPD. Copd 6: 121-129.

62. Pitta F, Takaki MY, Oliveira NH, Sant'anna TJ, Fontana AD, et al. (2008) Relationship between pulmonary function and physical activity in daily life in patients with COPD. Respir Med 102: 1203-1207. 
63. Cohen MD, Cutaia M (2010) A Novel Approach to Measuring Activity in Chronic Obstructive Pulmonary Disease: using 2 activity monitors to classify daily activity. J Cardiopulm Rehabil Prev 30: 186194.

64. Matthews CE, Ainsworth BE, Thompson RW, Bassett DR, Jr. (2002) Sources of variance in daily physical activity levels as measured by an accelerometer. Med Sci Sports Exerc 34: 1376-1381.

65. Cheung VH, Gray L, Karunanithi M (2011) Review of accelerometry for determining daily activity among elderly patients. Arch Phys Med Rehabil 92: 998-1014.

66. de Bruin ED, Hartmann A, Uebelhart D, Murer K, Zijlstra W (2008) Wearable systems for monitoring mobility-related activities in older people: a systematic review. Clin Rehabil 22: 878-895.

67. Coronado M, Janssens JP, de Muralt B, Terrier P, Schutz Y, et al. (2003) Walking activity measured by accelerometry during respiratory rehabilitation. J Cardiopulm Rehabil 23: 357-364.

68. Schonhofer B, Ardes P, Geibel M, Kohler D, Jones PW (1997) Evaluation of a movement detector to measure daily activity in patients with chronic lung disease. Eur Respir J 10: 2814-2819.

69. Belza B, Steele BG, Hunziker J, Lakshminaryan S, Holt L, et al. (2001) Correlates of physical activity in chronic obstructive pulmonary disease. Nurs Res 50: 195-202.

70. Steele BG, Holt L, Belza B, Ferris S, Lakshminaryan S, et al. (2000) Quantitating physical activity in COPD using a triaxial accelerometer. Chest 117: 1359-1367.

71. Hamilton MT, Hamilton DG, Zderic TW (2007) Role of low energy expenditure and sitting in obesity, metabolic syndrome, type 2 diabetes, and cardiovascular disease. Diabetes 56: 2655-2667.

72. Owen N, Healy GN, Matthews CE, Dunstan DW (2010) Too much sitting: the population health science of sedentary behavior. Exerc Sport Sci Rev 38: 105-113.

73. Langer D, Gosselink R, Sena R, Burtin C, Decramer M, et al. (2009) Validation of two activity monitors in patients with COPD. Thorax 64: 641-642.

74. Partridge MR, Karlsson N, Small IR (2009) Patient insight into the impact of chronic obstructive pulmonary disease in the morning: an internet survey. Curr Med Res Opin 25: 2043-2048.

75. Kavanagh JJ, Menz HB (2008) Accelerometry: a technique for quantifying movement patterns during walking. Gait Posture 28: 1-15.

76. Zijlstra W (2004) Assessment of spatio-temporal parameters during unconstrained walking. Eur J Appl Physiol 92: 39-44.

77. Yoshino K, Motoshige T, Araki T, Matsuoka K (2004) Effect of prolonged free-walking fatigue on gait and physiological rhythm. J Biomech 37: 1271-1280.

78. Helbostad JL, Leirfall S, Moe-Nilssen R, Sletvold O (2007) Physical fatigue affects gait characteristics in older persons. J Gerontol A Biol Sci Med Sci 62: 1010-1015.

79. Verghese J, Holtzer R, Lipton RB, Wang C (2009) Quantitative gait markers and incident fall risk in older adults. J Gerontol A Biol Sci Med Sci 64: 896-901.

80. Moe-Nilssen R, Helbostad JL (2005) Interstride trunk acceleration variability but not step width variability can differentiate between fit and frail older adults. Gait Posture 21: 164-170.

81. Menz HB, Lord SR, Fitzpatrick RC (2003) Age-related differences in walking stability. Age Ageing 32 : 137-142.

82. Jorgensen NR, Schwarz P, Holme I, Henriksen BM, Petersen L, et al. (2007) The prevalence of osteoporosis in patients with chronic obstructive pulmonary disease: a cross sectional study. Respir Med 101: 177-185.

83. Smith MD, Chang AT, Seale HE, Walsh JR, Hodges PW (2010) Balance is impaired in people with chronic obstructive pulmonary disease. Gait Posture 31: 456-460.

84. Roig M, Eng JJ, Maclntyre DL, Road JD, FitzGerald JM, et al. (2011) Falls in people with chronic obstructive pulmonary disease: an observational cohort study. Respir Med 105: 461-469.

85. Hausdorff JM, Rios DA, Edelberg HK (2001) Gait variability and fall risk in community-living older adults: a 1-year prospective study. Arch Phys Med Rehabil 82: 1050-1056.

86. Marquis N, Debigare R, Bouyer L, Saey D, Laviolette L, et al. (2009) Physiology of Walking in Patients with Moderate to Severe Chronic Obstructive Pulmonary Disease. Med Sci Sports Exerc 41: 15401548. 
87. Vaes AW, Wouters EF, Franssen FM, Uszko-Lencer NH, Stakenborg KH, et al. (2011) Task-related oxygen uptake during domestic activities of daily life in patients with COPD and healthy elderly subjects. Chest 140: 970-979.

88. Dolmage TE, Maestro L, Avendano MA, Goldstein RS (1993) The ventilatory response to arm elevation of patients with chronic obstructive pulmonary disease. Chest 104: 1097-1100.

89. Breslin EH (1992) Dyspnea-limited response in chronic obstructive pulmonary disease: reduced unsupported arm activities. Rehabil Nurs 17: 12-20.

90. Baarends EM, Schols AM, Slebos DJ, Mostert R, Janssen PP, et al. (1995) Metabolic and ventilatory response pattern to arm elevation in patients with COPD and healthy age-matched subjects. Eur Respir J 8: 1345-1351.

91. Franssen FM, Broekhuizen R, Janssen PP, Wouters EF, Schols AM (2005) Limb muscle dysfunction in COPD: effects of muscle wasting and exercise training. Med Sci Sports Exerc 37: 2-9.

92. Franssen FM, Wouters EF, Baarends EM, Akkermans MA, Schols AM (2002) Arm mechanical efficiency and arm exercise capacity are relatively preserved in chronic obstructive pulmonary disease. Med Sci Sports Exerc 34: 1570-1576.

93. Clark CJ, Cochrane LM, Mackay E, Paton B (2000) Skeletal muscle strength and endurance in patients with mild COPD and the effects of weight training. Eur Respir J 15: 92-97.

94. Gea JG, Pasto M, Carmona MA, Orozco-Levi M, Palomeque J, et al. (2001) Metabolic characteristics of the deltoid muscle in patients with chronic obstructive pulmonary disease. Eur Respir J 17: 939945.

95. Zhou H, Hu H, Tao Y (2006) Inertial measurements of upper limb motion. Med Biol Eng Comput 44: 479-487.

96. Coley B, Jolles BM, Farron A, Aminian K (2009) Detection of the movement of the humerus during daily activity. Med Biol Eng Comput 47: 467-474.

97. Coley B, Jolles BM, Farron A, Bourgeois A, Nussbaumer F, et al. (2007) Outcome evaluation in shoulder surgery using 3D kinematics sensors. Gait Posture 25: 523-532.

98. Cutti AG, Giovanardi A, Rocchi L, Davalli A, Sacchetti R (2008) Ambulatory measurement of shoulder and elbow kinematics through inertial and magnetic sensors. Med Biol Eng Comput 46: 169-178.

99. Brodie MA, Walmsley A, Page W (2008) Dynamic accuracy of inertial measurement units during simple pendulum motion. Comput Methods Biomech Biomed Engin 11: 235-242.

100. Hyde RA, Ketteringham LP, Neild SA, Jones RS (2008) Estimation of upper-limb orientation based on accelerometer and gyroscope measurements. IEEE Trans Biomed Eng 55: 746-754.

101. Preece SJ, Goulermas JY, Kenney LPJ, Howard D, Meijer K, et al. (2009) Activity identification using body-mounted sensors - a review of classification techniques. Physiological Measurement 30: R1R33.

102. Criner GJ, Celli BR (1988) Effect of unsupported arm exercise on ventilatory muscle recruitment in patients with severe chronic airflow obstruction. Am Rev Respir Dis 138: 856-861.

103. Owens GR, Thompson FE, Sciurba FC, Robertson R, Metz KF, et al. (1988) Comparison of arm and leg ergometry in patients with moderate chronic obstructive lung disease. Thorax 43: 911-915.

104. Schasfoort FC, Bussmann JB, Martens WL, Stam HJ (2006) Objective measurement of upper limb activity and mobility during everyday behavior using ambulatory accelerometry: the upper limb activity monitor. Behav Res Methods 38: 439-446.

105. Zhou H, Stone T, Hu H, Harris N (2008) Use of multiple wearable inertial sensors in upper limb motion tracking. Med Eng Phys 30: 123-133.

106. Gosselink R, Troosters T, Decramer M (2000) Distribution of muscle weakness in patients with stable chronic obstructive pulmonary disease. J Cardiopulm Rehabil 20: 353-360. 

Chapter 2

Problematic activities of daily life are weakly associated with clinical characteristics in COPD

Janneke Annegarn, Kenneth Meijer, Valeria Lima Passos, Katharina Stute, Joze Wiechert, Hans H. C. M. Savelberg, Annemie M. W. J. Schols, Emiel F. M. Wouters and Martijn A. Spruit.

J Am Med Dir Assoc, 2012. 13: 284-290. 


\begin{abstract}
Problematic Activities of Daily Life (ADLs) can be the main reason to refer patients with Chronic Obstructive Pulmonary Disease (COPD) for pulmonary rehabilitation. To date, information on problematic ADLs and their clinical correlates in COPD remain scarce. This retrospective chart review aimed to identify the most prevalent self-reported problematic ADLs in COPD patients, determine performance and satisfaction scores of these problematic ADLs and explore the association between 13 clinical determinants and four problematic ADL domains. 820 COPD patients entering pulmonary rehabilitation assessment were included. Self-reported problematic ADLs were assessed using the Canadian Occupational Performance Measure (COPM), administered by occupational therapists in the form of a semi-structured

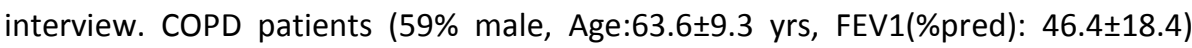
reported 2999 problematic ADLs. The most prevalent problematic ADLs were walking (68\%), stair climbing (35\%) and cycling (30\%). Moreover, 30\% of the patients reported 'not able to do any of the scored problematic $A D L^{\prime}$ ' and $44 \%$ were 'not satisfied at all with the performance of any of the scored problematic $A D L$ '. Significant but weak associations were found between clinical determinants (e.g., physical and psychosocial) and problematic ADLs. The lack of a strong association between problematic ADLs and clinical determinants, emphasizes the need of individualized assessment of these ADLs to allow tailored intervention.
\end{abstract}




\section{Introduction}

Self-reported function meaningfully reflects physiology in moderately to severely disabled older adults [1]. Patients with chronic obstructive pulmonary disease (COPD) experience a progressive reduction in the ability to perform Activities of Daily Life (ADLs) during the course of their disease [2]. Patients who are dissatisfied with the ability to perform ADLs are often the ones referred to pulmonary rehabilitation [2].

Patients' self perceived performance and satisfaction of problematic ADLs can be measured using patient reported questionnaires. Patient reported questionnaires show good validity for measuring general problematic ADLs, but poorly identify patients' individual needs [3]. An alternative approach is offered by interviews. Although these can be time consuming, they enable an exploration of the individual problematic ADLs [4,5]. The Canadian Occupational Performance Measure (COPM) is such an individualized outcome measure [6]. which can be used reliably in patients with activity limitations and participation restrictions (e.g. COPD, chronic pain, hand injury, multiple sclerosis, diabetes mellitus) $[7,8]$. Indeed the COPM has been recommended for use during the assessment of pulmonary rehabilitation programs for patients with COPD $[9,10]$. However an overview of the distribution of problematic ADLs in a large cohort of COPD patients is currently lacking.

The World Health Organization has developed the framework International Classification of Functioning, Disability and Health (ICF) to describe and analyze the functional consequences of various health conditions [11]. ICF does not take into account that the importance and satisfaction connected to the ability to perform various ADLs may vary among patients. There is limited information on whether and to what extent objective clinical determinants are associated with the subjective functional consequences of COPD. For example, it has been reported that female COPD patients perceive more loss of functional performance within 'home management' compared to male COPD patients [12]. Moreover, given previous reports, it seems reasonable to hypothesize that also living alone $[13,14]$, being younger $[15,16]$, having a higher degree of airflow limitation [16], receiving long-term oxygen therapy (LTOT) $[5,17]$, having a poor functional exercise performance (lower 6minute walking distance) $[12,18]$, not using a walking aid (e.g., rollator or cane) [19], depletion of fat-free mass [20], increased fat mass [21], lower scores on variables reflecting dyspnea $[18,22]$, psychological status $[14,18]$ and/or health status $[18,23]$ may decrease performance and satisfaction of problematic ADLs in COPD. Unfortunately, available studies are limited by sample size and/or focused on pre-specified 
ADLs. Nevertheless, the identification of clinical determinants of problematic ADLs may allow clinicians to better monitor and restore the impaired functional performance and/or satisfaction in patients with COPD.

Therefore, the aim of the current study was threefold: 1 : to identify the most prevalent problematic ADLs in patients with COPD; 2: to determine performance and satisfaction scores for these ADLs; and 3: to explore the association between clinical determinants and problematic ADLs.

\section{Methods}

\section{Participants}

Data were extracted from the records of 1102 clinically stable patients with the diagnosis of 'COPD' who were evaluated at $\mathrm{CIRO}+$, a centre of excellence for chronic organ failure in Horn (the Netherlands), for rehabilitation eligibility between January 1, 2005 and April 1, 2009. Of these records, 820 met the following inclusion criteria: all necessary data present, a forced expiratory (FEV1)/forced vital capacity (FVC) ratio of $\leq 0.7$, and not representing a repeat admission for the same patient. These retrospective analyses are not institutional review board exempt due to the use of de-identified, preexisting data.

\section{Measures}

As part of routine assessment for rehabilitation eligibility patients underwent a 6minute walking test in accordance with the guidelines of the American Thoracic Society [24], including a practice walk. The best six minute walk distance (6MWD) was expressed as a percentage of the predicted values [25]. Patients also underwent routine post-bronchodilator spirometry, physical examination and medical history assessment [26]. Length and weight were assessed to obtain the body mass index (BMI, body weight in kilograms divided by squared height in meters, $\mathrm{kg} / \mathrm{m}^{2}$ ), and a bio-electrical impedance analysis was used (Bodystat 1500, Euromedix, Leuven) to determine the fat-free mass index (FFMI) and the fat mass index (FMI) using disease-specific equations [27]. Patients were identified as current smokers when arterial carboxihemoglobin $(\mathrm{HbCO}) \geq 2 \%$. All patients completed the Medical Research Council (MRC) dyspnea scale, the St. George's Respiratory Questionnaire (SGRQ) and the Hospital Anxiety and Depression Scale (HADS) [28]. Moreover, patients underwent an intake by an occupational therapist, including the COPM [7].

The COPM is administered in the form of a semi-structured interview, in which the patient is encouraged to identify and discuss specific problematic ADLs. Previous to quantitative analyses $[29,30]$, problematic ADLs were simplified and categorized 
into four COPM domains: 'self-care,' 'productivity', 'leisure' and 'mobility' [6]. Mobility-related problematic ADLs are usually part of the COPM domain 'self-care' [31]. However, in COPD patients mobility related problematic ADLs were expected to be highly prevalent [12] and therefore analyzed separately. Next, patients identified their perception of how well they were performing the problematic ADL (performance score) and how satisfied they were with this level of performance (satisfaction score). These scores were ascertained by using the cue cards to identify a score between 1 ('not able to do it' or 'not at all satisfied', respectively) to 10 points ('able to do it extremely well' or 'extremely satisfied').

Statistics

Mean and Standard Deviation (SD) are displayed for quantitative variables with symmetric distributions and percentages for categorical variables. A limited amount of patients with GOLD stage $1(n=48)$ and MRC dyspnea grade $1(n=20)$ were evaluated at CIRO+. Therefore GOLD stages 1 and 2 were combined for the analyses. The same was done for MRC dyspnea grades 1 and 2. The comparisons were conducted with one-way analysis of variance or Chi-Square tests as appropriate. Adjustment for multiple testing was conducted via False Discovery Rate (FDR). Results were considered statistically significant when FDR p-value was $<0.05$. The 10 most prevalent problematic ADLs were described for the whole group and after stratification for sex, age ( $<65$ or $\geq 65$ years) and GOLD stage. Age was dichotomized according to the mean age as reported in the Cochrane review of Lacasse and colleagues [32].

Logistic regression analyses were conducted to test whether reporting (yes/no) any of the 4 identified problematic COPM domains ('self-care', 'productivity', 'leisure' and 'mobility') was associated with the following clinical determinants previously reported to affect ADLs in COPD patients: age (years), sex, living status (alone/together), $\mathrm{FEV}_{1}$ (\% predicted), FFMI $\left(\mathrm{kg} / \mathrm{m}^{2}\right), \mathrm{FMI}\left(\mathrm{kg} / \mathrm{m}^{2}\right), 6 \mathrm{MWD}(\%$ predicted), walking aid use (yes/no), receiving long term oxygen therapy (LTOT) (yes/no), MRC dyspnea grade, $\mathrm{SGRQ}_{\text {total }}$ score, anxiety score and depression score (both HADS). Logistic models were fitted for each of the 4 COPM domains separately. The Area Under the Curve (AUC) of the receiver operating characteristic (ROC) curve was calculated as a measure of the final models predictive values. This same set of predictors was tested in their association with performance and satisfaction scores via multiple Ordinary Least Squares (OLS) regression models. Performance and satisfaction scores were averaged over repeated measures per patient within each COPM domain separately. These mean scores were used as outcome variable in the OLS regression models. For both, OLS and logistic regression analyses, multicollinearity tests were carried out in advance and the variables were retained in the models, if the variance inflation factor was smaller than 3.0. A topdown procedure was handled for the selection of the final model variables. For all tests a statistical significance level of $\leq 5 \%$ was handled. Data analyses were per- 
formed with Statistical Package for Social Sciences (SPSS) 15.0 for Windows (SPSS Inc., Chicago, IL, USA).

\section{Results}

\section{Patients' characteristics.}

The current cohort consisted mostly of elderly male COPD patients, with generally a normal body composition, and a poor functional exercise performance. About onethird of the patients used a walking aid. As expected, patients in GOLD stage 4 had the worst mean 6-minute walking distance, also after correction for confounding variables, like height, body weight, age and sex [25]. Furthermore, mean SGRQ ${ }_{\text {total }}$ scores were significantly higher (=worse) among GOLD 4 patients (table 2.1).

\section{Problematic $A D L s$}

820 patients reported 2999 problematic ADLs. 743 patients scored $\geq 2$ problematic ADLs. The COPM domain 'mobility' was most frequently scored (86\%) (performance score: $4.2 \pm 1.7$ points, satisfaction score: $3.5 \pm 2.0$ points) followed by 'productivity' (56\%) (performance score: $4.3 \pm 1.8$ points, satisfaction score: $3.7 \pm 2.0$ points) , 'selfcare' (55\%) (performance score: $4.8 \pm 1.6$ points, satisfaction score: $4.2 \pm 2.1$ points) and 'leisure' (46\%) (performance score: $3.9 \pm 2.0$ points, satisfaction score: $3.3 \pm 2.2$ points).

Top-10 problematic ADLs.

The three most prevalent problematic ADLs were walking, Stair climbing and cycling (table 2.2). Walking remained the most prevalent problematic ADL after stratification for sex, age (<65 vs $\geq 65$ years) and/or GOLD stage (shown in table 2.3, table 2.4 and table 2.5$)$.

A higher proportion of GOLD stage 4 patients scored problematic ADLs within the COPM domain 'self-care' compared to GOLD stages $1 / 2$ and $3(p<0.001)$. In contrast, the proportion of GOLD stage 4 patients with problematic ADLs within the COPM domain 'productivity' was significantly lower ( $p=0.037$; figure $2.1 \mathrm{a}$ ). No significant differences were found for the COPM domains 'leisure' $(p=0.485)$ and 'mobility' $(p=0.060)$. 
Table 2.1: Patients' characteristics

\begin{tabular}{|c|c|c|c|c|c|}
\hline & $\begin{array}{c}\text { Total } \\
(\mathrm{N}=820)\end{array}$ & $\begin{array}{c}\text { GOLD 1/2 } \\
(\mathrm{N}=289)\end{array}$ & $\begin{array}{l}\text { GOLD } 3 \\
(\mathrm{~N}=234)\end{array}$ & $\begin{array}{l}\text { GOLD } 4 \\
(N=297)\end{array}$ & $\begin{array}{c}\text { FDR } \\
\text { p-value }\end{array}$ \\
\hline Sex (\% male) & 59 & 51.2 & $65.4^{*}$ & $61.6^{*}$ & 0.0034 \\
\hline Age (yrs) & $63.6 \pm 9.3$ & $63.8 \pm 9.5$ & $63.0 \pm 9.5$ & $63.8 \pm 9.0$ & 0.571 \\
\hline FEV1 (L) & $1.3 \pm 0.6$ & $1.75 \pm 0.53$ & $1.1 \pm 0.3^{*}$ & $0.86 \pm 0.3^{*} \#$ & $<0.0001$ \\
\hline FEV1 (\%pred) & $46.4 \pm 18.4$ & $65.9 \pm 12.5$ & $40.1 \pm 5.7^{*}$ & $32.3 \pm 12.4 * \#$ & $<0.0001$ \\
\hline Tiffeneau index (\%) & $39.6 \pm 11.7$ & $49.2 \pm 9.4$ & $36.9 \pm 7.8^{*}$ & $32.4 \pm 10.0 * \#$ & $<0.0001$ \\
\hline pO2 (kPa) & $9.4 \pm 1.5$ & $9.8 \pm 1.4$ & $9.6 \pm 1.1$ & $8.8 \pm 1.8^{*} \#$ & $<0.0001$ \\
\hline $\mathrm{pCO} 2(\mathrm{kPa})$ & $5.4 \pm 0.9$ & $5.0 \pm 0.6$ & $5.2 \pm 0.5^{*}$ & $5.9 \pm 1.2^{*} \#$ & $<0.0001$ \\
\hline Current smoker (\%) & 41.8 & 43.6 & 43.6 & 38.7 & 0.4148 \\
\hline LTOT (\%) & 21.7 & 0 & 0 & $59.9 * \#$ & $<0.0001$ \\
\hline Charlson score (points) & $1.7 \pm 1.2$ & $1.6 \pm 1.0$ & $1.7 \pm 1.1$ & $1.8 \pm 1.4^{*}$ & 0.07 \\
\hline MRC dyspnea grade & $3.4 \pm 1.1$ & $2.9 \pm 1.1$ & $3.4 \pm 1.1^{*}$ & $4.0 \pm 1.04^{*} \#$ & $<0.0001$ \\
\hline $\mathrm{BMI}\left(\mathrm{kg} / \mathrm{m}^{2}\right)$ & $25.2 \pm 5.0$ & $25.9 \pm 4.7$ & $24.7 \pm 4.8^{*}$ & $25.0 \pm 5.3^{*}$ & 0.0115 \\
\hline FFMI $\left(\mathrm{kg} / \mathrm{m}^{2}\right)$ & $16.3 \pm 2.2$ & $16.5 \pm 2.1$ & $16.3 \pm 2.1$ & $16.2 \pm 2.3$ & 0.1956 \\
\hline FMI $\left(\mathrm{kg} / \mathrm{m}^{2}\right)$ & $8.9 \pm 3.4$ & $9.4 \pm 3.2$ & $8.4 \pm 3.4^{*}$ & $8.8 \pm 3.6^{*}$ & 0.0032 \\
\hline Living alone (\%) & 23.9 & 22.8 & 21.4 & 26.9 & 0.3119 \\
\hline 6MWD (m) & $431.7 \pm 125.3$ & $487.3 \pm 105.0$ & $451.9 \pm 113.7^{*}$ & $361.7 \pm 119.4^{*} \#$ & $<0.0001$ \\
\hline 6MWD (\%pred) & $79.8 \pm 21.9$ & $91.9 \pm 17.5$ & $81.4 \pm 18.0^{*}$ & $66.9 \pm 21.5 * \#$ & $<0.0001$ \\
\hline Walking aid (\%) & 29.3 & 9.3 & 12.4 & $62.0 * \#$ & $<0.0001$ \\
\hline$S G R Q_{\text {Impact }}$ (points) & $43.8 \pm 18.6$ & $38.3 \pm 17.9$ & $43.4 \pm 17.9 *$ & $49.5 \pm 18.2 * \#$ & $<0.0001$ \\
\hline$S_{G R Q_{\text {Activity }}}$ (points) & $72.1 \pm 18.7$ & $65.1 \pm 19.4$ & $70.0 \pm 17.9^{*}$ & $80.4 \pm 14.9 * \#$ & $<0.0001$ \\
\hline $\mathrm{SGRQ}_{\text {symptom }}$ (points) & $60.1 \pm 20.5$ & $56.2 \pm 21.6$ & $61.1 \pm 20.2^{*}$ & $63.2 \pm 19.1^{*}$ & 0.0003 \\
\hline $\mathrm{SGRQ}_{\text {total }}$ (points) & $55.1 \pm 16.2$ & $49.4 \pm 16.2$ & $54.4 \pm 15.6^{*}$ & $61.1 \pm 14.4^{*} \#$ & $<0.0001$ \\
\hline Anxiety score (points) & $7.6 \pm 4.4$ & $7.2 \pm 3.9$ & $7.4 \pm 4.4$ & $8.0 \pm 4.8^{*}$ & 0.0579 \\
\hline Depression score (points) & $6.9 \pm 4.0$ & $6.4 \pm 3.8$ & $6.7 \pm 3.8$ & $7.6 \pm 4.2^{*} \#$ & 0.0006 \\
\hline
\end{tabular}

*=different from GOLD 1+2; \# = different from GOLD 3; 
Table 2.2: Top 10 problematic ADLs for all patients

\begin{tabular}{lllll}
\hline \multicolumn{1}{l}{ Activity } & All patients (N=820) & \\
\hline 1 & Walking & $\%$ & $\mathrm{P}$ & $\mathrm{S}$ \\
2 & Stair climbing & 68.3 & 4.25 & 3.6 \\
3 & Cycling & 35 & 4.28 & 3.47 \\
4 & Showering & 30 & 3.83 & 3.42 \\
5 & Gardening & 20.1 & 4.9 & 4.25 \\
6 & Cleaning the floor & 17.6 & 4.16 & 3.73 \\
7 & Dress and undress & 12.2 & 4.62 & 4.05 \\
8 & Sports & 11 & 4.94 & 4.39 \\
9 & Social activities & 8.9 & 3.03 & 3.08 \\
10 & Activities with (grand) children & 7.8 & 3.66 & 2.94 \\
\hline
\end{tabular}

Abbreviations: $\mathrm{P}=$ mean performance score (points), $\mathrm{S}=$ mean satisfaction score (points).

Table 2.3: Top 10 problematic ADLs for all patients after stratification for gender

\begin{tabular}{|c|c|c|c|c|c|c|c|}
\hline \multicolumn{4}{|c|}{ Men (N=484) } & \multicolumn{4}{|c|}{ Women (N=336) } \\
\hline Activity & $\%$ & $P$ & $S$ & Activity & $\%$ & $P$ & $S$ \\
\hline Walking & 69.8 & 4.31 & 3.85 & Walking & 66.1 & 4.18 & 3.22 \\
\hline Stair climbing & 35.7 & 4.29 & 3.59 & Stair climbing & 33.9 & 4.24 & 3.29 \\
\hline Cycling & 32.9 & 3.94 & 3.53 & Cycling & 25.9 & 3.68 & 3.25 \\
\hline Gardening & 22.9 & 4.1 & 3.7 & Showering & 18.8 & 4.91 & 3.92 \\
\hline Showering & 21.1 & 4.9 & 4.45 & Cleaning the floor & 16.4 & 4.38 & 3.74 \\
\hline $\begin{array}{l}\text { Dress and un- } \\
\text { dress }\end{array}$ & 12.6 & 4.86 & 4.57 & Making the bed & 11.3 & 4.32 & 3.55 \\
\hline $\begin{array}{l}\text { Working around } \\
\text { the house }\end{array}$ & 10.1 & 3.76 & 3.26 & Light work in the house & 10.7 & 4.03 & 3.67 \\
\hline $\begin{array}{l}\text { Cleaning the } \\
\text { floor }\end{array}$ & 9.3 & 4.9 & 4.42 & Preparing food & 10.1 & 3.72 & 3.35 \\
\hline $\begin{array}{l}\text { Carrying / drag- } \\
\text { ging }\end{array}$ & 9.1 & 4.36 & 3.8 & Social activities & 10.1 & 3.76 & 2.62 \\
\hline Sports & 8.9 & 3.43 & 3.74 & Gardening & 9.8 & 4.33 & 3.82 \\
\hline
\end{tabular}

Abbreviations: $\mathrm{P}=$ mean performance score (points), $\mathrm{S}=$ mean satisfaction score (points). 
Table 2.4: Top 10 problematic ADLs of men stratified for age and GOLD stage

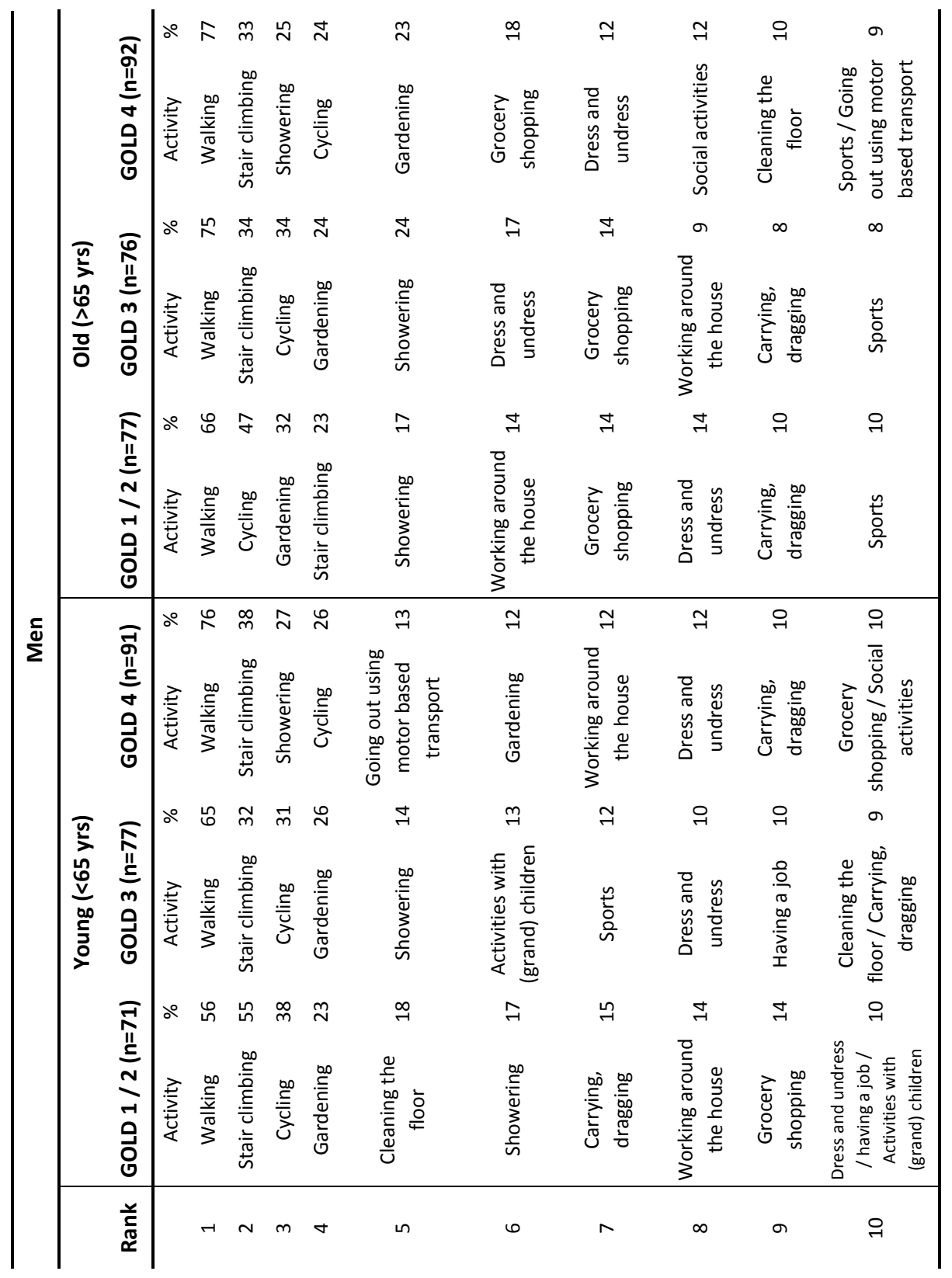


Table 2.5: Top 10 problematic ADLs of women stratified for age and GOLD stage

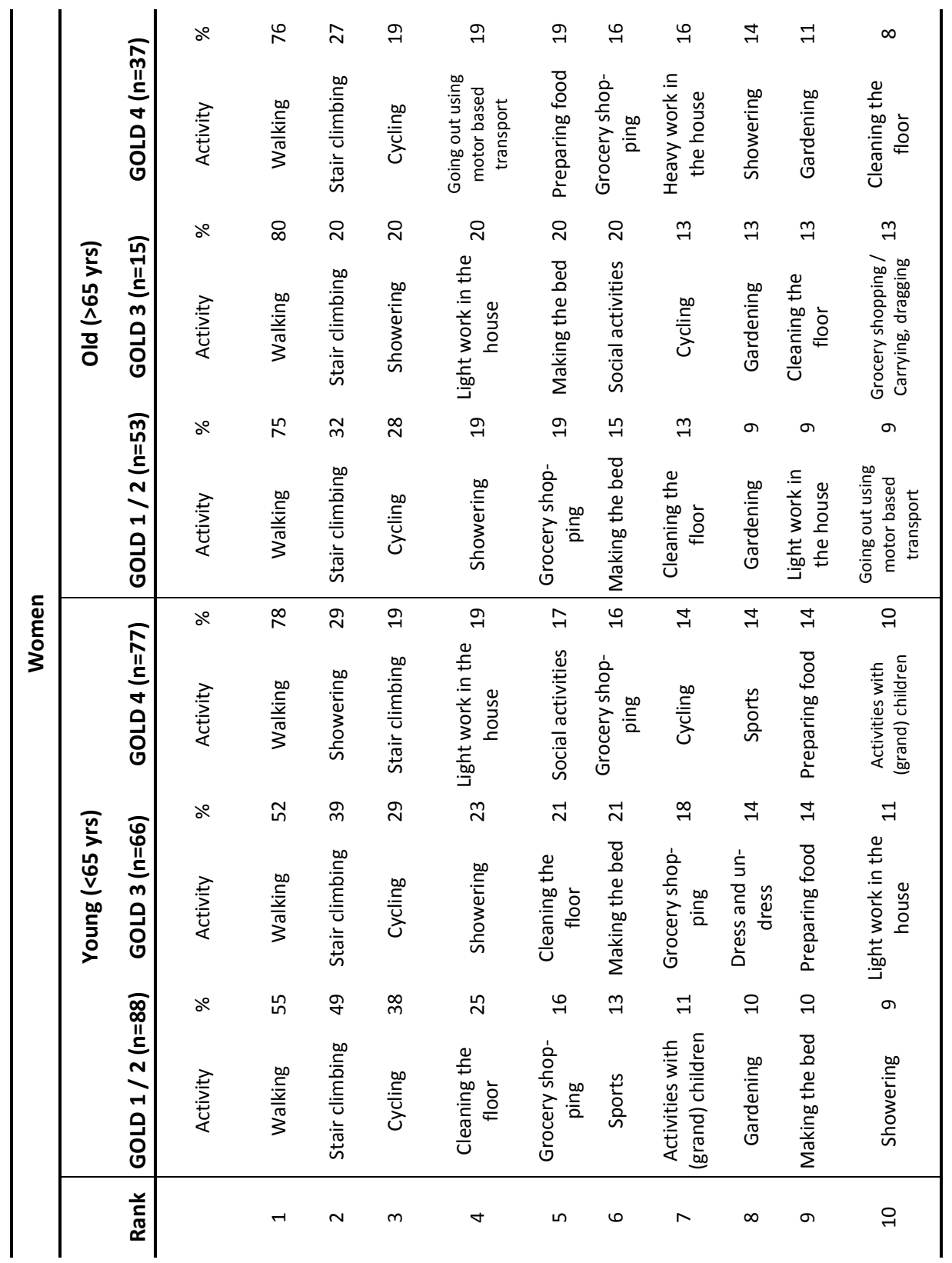


$80 \%$ of the patients with MRC grade 5 scored $\geq 1$ problematic ADL on the COPM domain 'self-care', while this was significantly lower in the patients with MRC grade 1 or 2 ( $26 \%$; $p<0.001)$. Moreover, the proportion of patients with $\geq 1$ problematic ADL within the COPM domain 'mobility' was lowest in patients with MRC grade 1 or 2 , but was still highly prevalent $(77 \%)(p=0.001$; Figure $1 \mathrm{~b})$. The COPM domains 'productivity' ( $p=0.389)$ and 'leisure' $(p=0.741)$ were not different amongst MRC dyspnea grades.
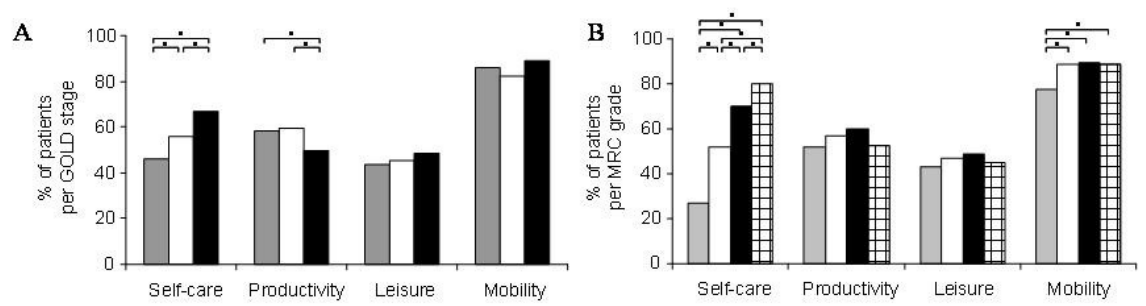

Figure 2.1a: \% of patients who scored within a specific COPM domain stratified for GOLD stage $1 / 2$ (grey, $\mathrm{n}=289$ ), 3 (white, $\mathrm{n}=234$ ) and 4 (black, $\mathrm{n}=297$ ). Figure $2.1 \mathrm{~b}$ : \% of patients who scored within a specific COPM domain stratified for MRC dyspnea grade 1/2 (grey, $n=190$ ), 3 (white, $n=277$ ), 4 (chequered, $\mathrm{n}=150$ ) and 5 (black, $\mathrm{n}=203$ ). ${ }^{*} \mathrm{p}<0.05$.

\section{Performance and satisfaction scores.}

The frequency distributions of performance and satisfaction points for each COPM domain after stratification for GOLD are summarized in figure 2.2. The proportion of patients scoring 1 point for performance or satisfaction was 6.5 and $22.5 \%$ for the COPM domain 'self-care', respectively; 14.1 and 27.9\% for 'productivity'; 27.1 and $39.1 \%$ for 'leisure'; and 17.7 and $33.6 \%$ for 'mobility'.

\section{Logistic regression analysis.}

Several clinical determinants were observed to significantly affect the odds of reporting problematic ADLs in any of the COPM domains. However, no uniform pattern of associations was found across the COPM domains. The identified predictors were poor to moderate in discriminating between COPD patients with and without $\geq 1$ problematic ADLs in the 4 COPD domains ( ROC $_{\text {AUC }}$ : 0.571-0.754, table 2.6).

Patients with a lower 6MWD, a higher MRC dyspnea grade or a higher total score on the SGRQ had higher odds to score $\geq 1$ problematic ADLs within the COPM domain 'self-care'. Patients who were younger, who were women, with a higher $\mathrm{FEV}_{1}$ or a higher total score on the SGRQ had higher odds to score $\geq 1$ problematic ADLs within the COPM domain 'productivity'. Patients who were men or scored higher on depression had higher odds to score $\geq 1$ problematic ADLs within the COPM domain 'leisure'. Patients with a higher MRC dyspnea grade had higher odds to score $\geq 1$ problematic ADLs within the COPM domain 'mobility' (table 2.6). 
A
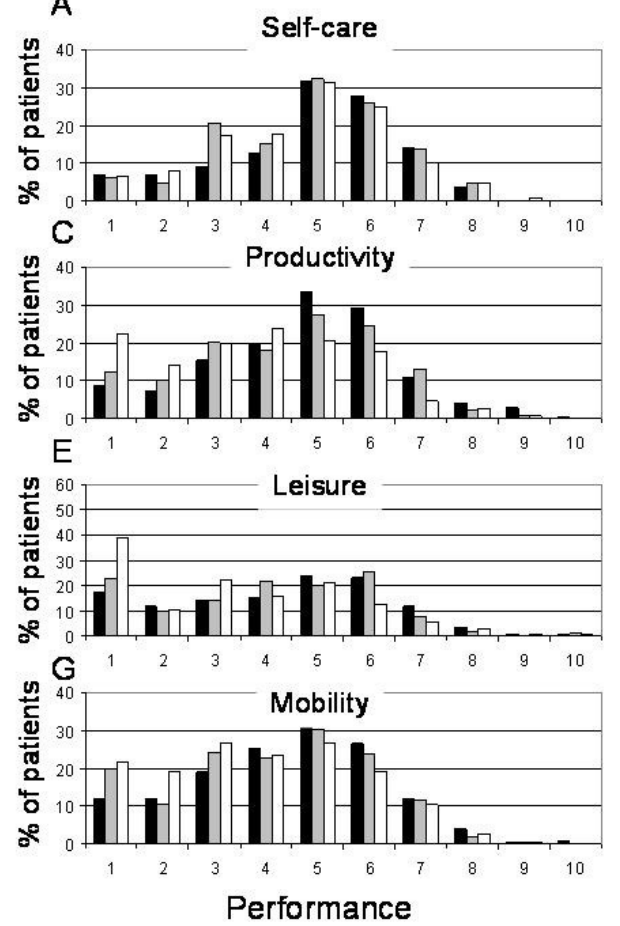

B
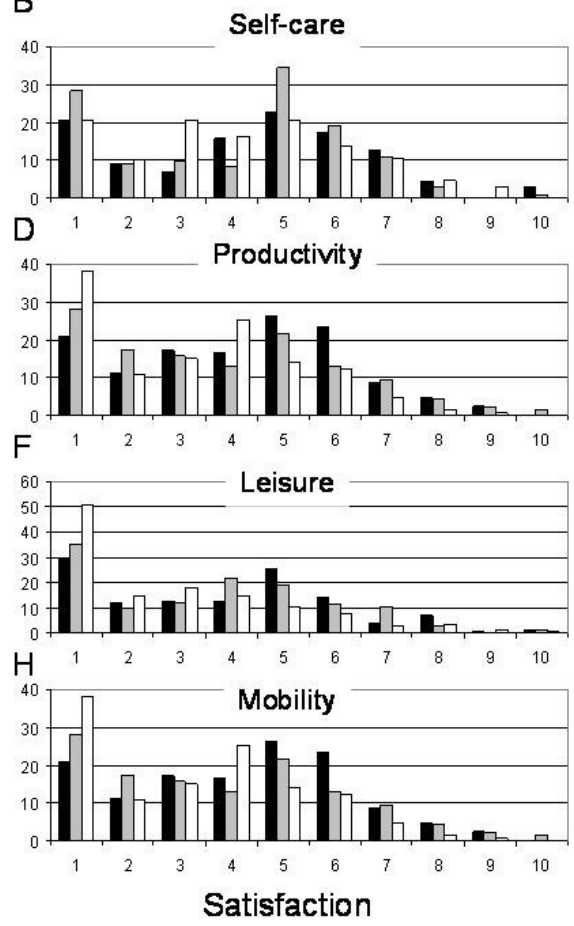

Figure 2.2: Proportion of patients after stratification for GOLD stages (GOLD stage $1 / 2=$ black, $3=$ grey and $4=$ white). As 743 patients scored $>1$ problematic ADL, the sum of $\%$ per GOLD stage was $>100 \%$. $2 A$ : proportion of patients scoring self-care performance (1 to 10 points). 2.2B: proportion of patients scoring self-care satisfaction ( 1 to 10 points). $2.22 \mathrm{C}$ : proportion of patients scoring productivity performance. 2.2D: proportion of patients scoring productivity satisfaction. 2.2E: proportion of patients scoring leisure performance. 2.2F: proportion of patients scoring leisure satisfaction. 2.2G: proportion of patients scoring mobility performance $2.2 \mathrm{H}$ : proportion of patients scoring mobility satisfaction.

\section{OLS regression analysis}

The results of the OLS regression models used to test for associations between clinical outcomes and the mean scores for performance and satisfaction for each COPM domain are summarised in table 2.7. Similar to the logistic analysis, the OLS analysis revealed that performance and satisfaction scores among different COPM domains are complex and cannot be predicted from the same set of predictors. No major predictor was found to influence all domains scores simultaneously. Despite significant associations, the models goodness of fit was poor ( $R^{2} 7.5$ to 14.6). 
Table 2.6: Odds ratio's (OR) of the model variables influencing reporting (yes, no) any of the 4 COPM domains - Logistic Regression

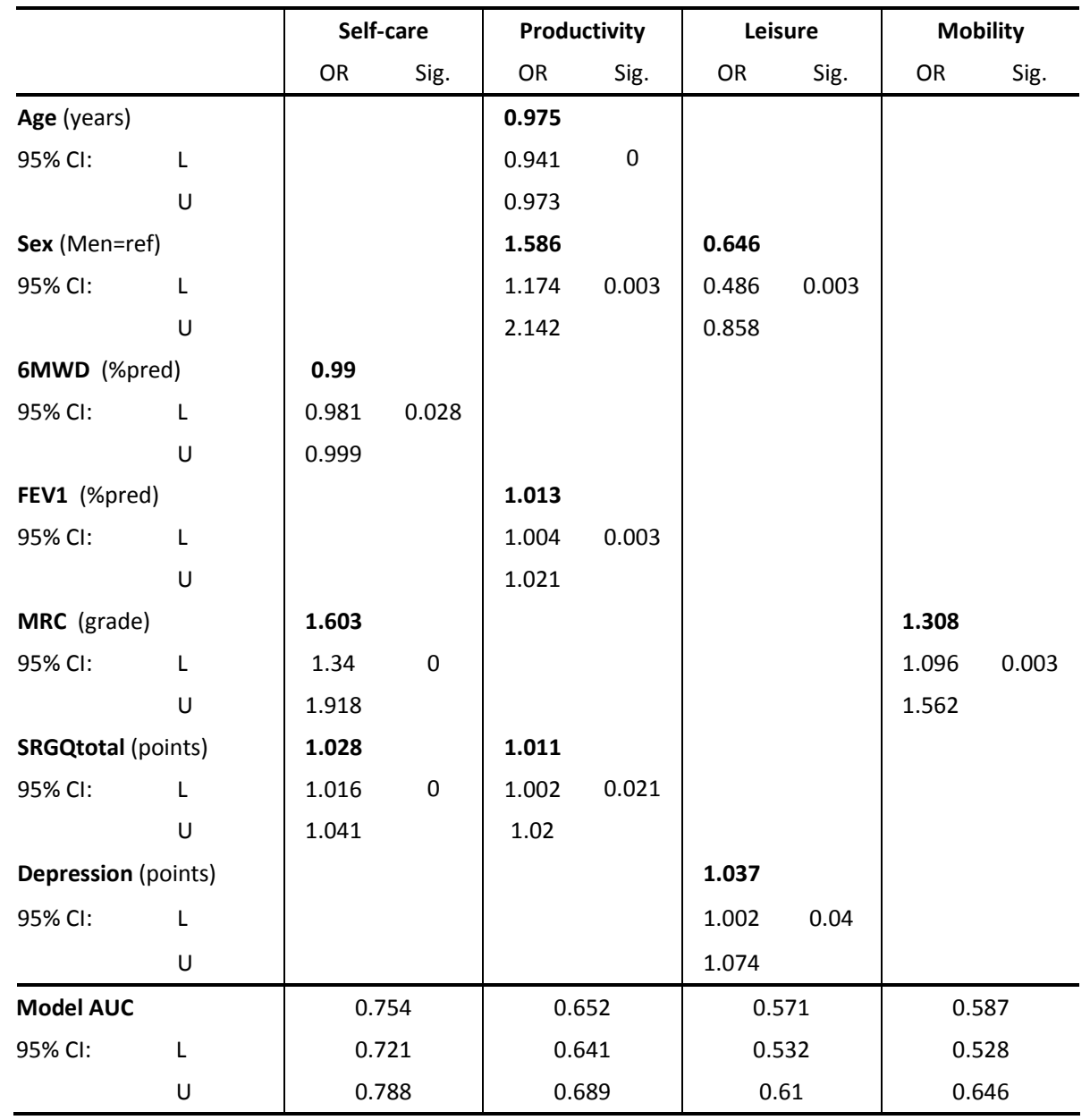

Abbreviations: $L=l o w e r, U=u p p e r$, ref=reference value Note: $O R$ are presented per unit increase of the independent variable; Effects of the following variables were also tested, but no statistical significance was detected: Living status, FFMI, FMI, anxiety score, receiving LTOT and using a walking aid. 
Table 2.7: Beta's ( $(S)$ of the model variables influencing performance (P) and satisfaction (S) of any of the 4 COPM domains - Linear Regression.

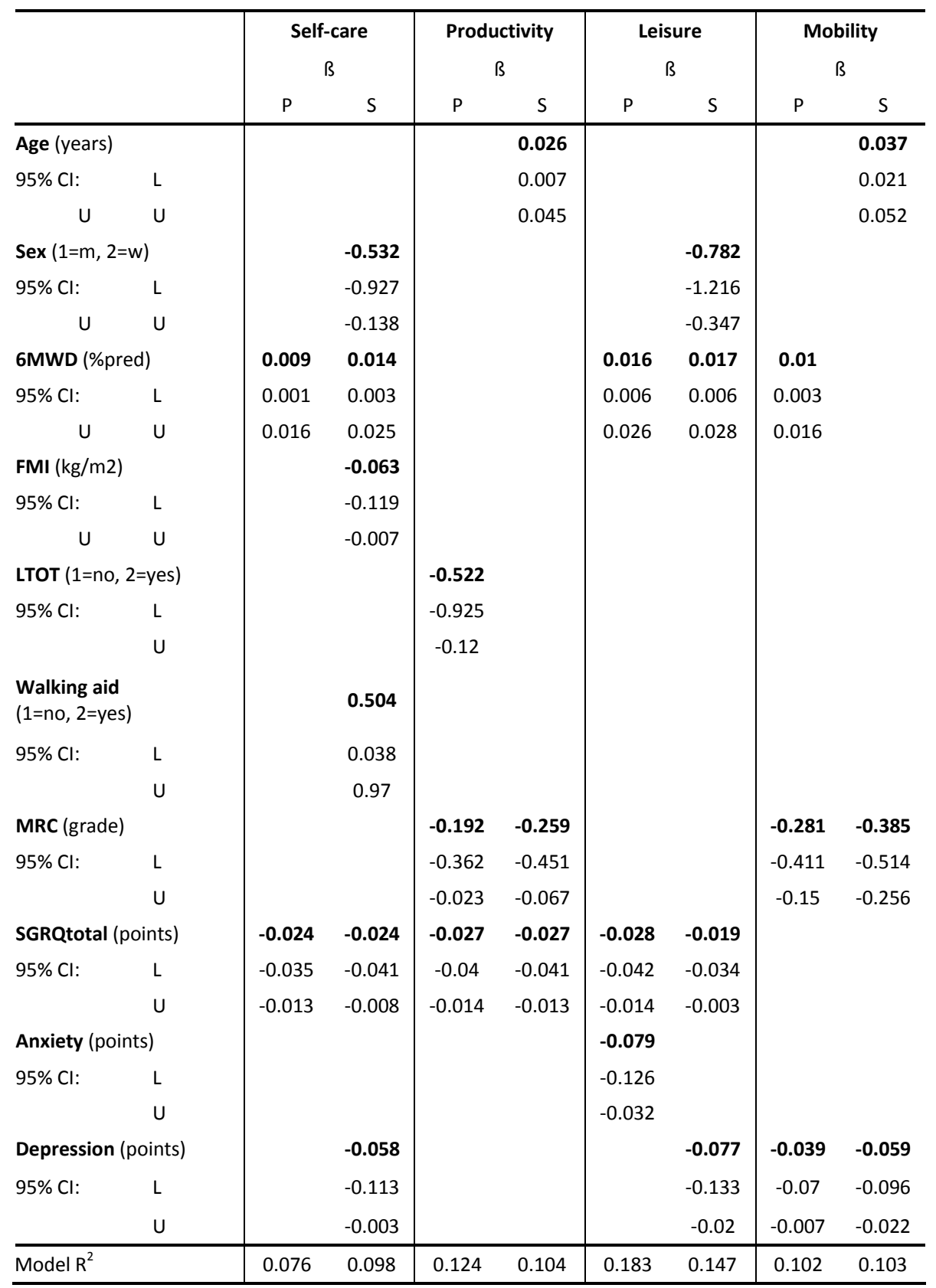

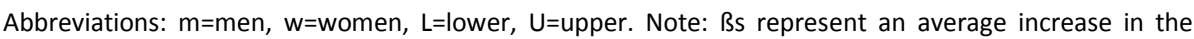
scores per unit increase in the independent variable; No statistical significance was detected for Living status, FEV1 and FFMI 


\section{Discussion}

This is the most comprehensive evaluation of problematic ADLs in COPD patients to date, in which a staggering range of 2999 problematic ADLs were reported. Walking was the most prevalent problematic ADL independent of age, sex or GOLD stage. Moreover, a huge proportion of patients were not satisfied at all with one or more problematic ADLs. Known clinical determinants of ADLs in COPD patients were only weakly associated with performance and satisfaction scores of problematic ADLs in the current analyses. Taken together, the wide range of problematic ADLs and the absence of strong association with clinical determinants emphasize the need of using interview based performance measures for identification of ADLs that an individual perceives as problematic to perform to allow tailored interventions.

The strength of this study relies in the inclusion of a large sample of COPD patients with different levels of disease severity. The individual problematic ADLs were assessed using a semi-structured interview measure, which is beneficial over standardized questionnaires as it does not focus on specific ADLs which may not be most important to the patient. Moreover, this study analyzed 13 clinical determinants previously reported to affect ADLs in COPD patients.

\section{Problematic $A D L s$}

Walking is most frequently reported to be problematic in daily life, which is in general agreement with small-sized studies that have described the importance of daily walking for COPD patients [4,5,33]. In addition, 6 out of the current top 10 of problematic ADLs correspond with previous findings [34]. All 4 COPM domains are represented in the top-10 of problematic activities, showing the diversity of the problematic ADLs that appear in COPD patients.

\section{Clinical correlates of problematic ADLS}

Identifying clinical determinants of problematic ADLs may allow clinicians to better monitor and restore the impaired functional performance and/or satisfaction in patients with COPD. In the present study, the degree of airflow was not associated with performance or satisfaction scores at all. This study is in alignment with previous findings showing that also patients with moderate COPD have significant impairment in ADLs [22]. Even though MRC dyspnea grade appeared to be somewhat more associated with problematic ADLs, both GOLD classification and MRC dyspnea grade are not discriminative enough to be used as a clinical tool for evaluating problematic ADLs of the individual patient. 
Better functional exercise performance, lower MRC dyspnea grade, lower $\mathrm{SGRQ}_{\text {total }}$ and lower depression score are associated with higher average performance and satisfaction scores in 2 or more COPM domains. In line with this, the same clinical values were previously also found to be associated with the London Chest ADL scale [18]. Additionally, Katz et al. found a positive association between the level of psychological distress and difficulty with ADLs [14].

Women have less problematic ADLs within the COPM domain 'leisure', while the opposite applies for the COPM domain 'productivity'. This corresponds to previous results on sex differences [12]. Age was negatively associated with scoring 1 or more problematic ADLs within the COPM domain 'productivity', which is probably a result of older patients being retired more frequently.

Although several clinical determinants were shown to significantly affect the odds of reporting any of the 4 COPM domains, the models predictive power was limited. Likewise, weak correlations were observed between clinical determinants and their identified perception of how well they are performing the problematic ADLs and how satisfied they are with this level of performance. Therefore, it may be speculated that some other factors not included in our models could improve their discriminative power (e.g., personal assistance [5] and self-esteem [33]). Most likely, the COPM provides additional clinical relevant information which cannot be predicted from other clinical determinants. Either way, it highlights the importance of assessing the problematic ADLs of individual patients.

\section{Methodological considerations}

This retrospective chart review focused on clinically stable COPD patients entering pulmonary rehabilitation, which might limit the external validity of the present findings. Statistical analyses were conducted only for patients with complete data $(n=820)$ as missing values were assumed to be at random. In this study a total of 2999 problematic ADLs were reported. Similar problematic ADLs were reported by the patients in many different ways. In the process of summarizing the overall picture by stipulating major general categories, we noticed some difficulties. For example, some patients reported their ADLs very specific (e.g. problems with showering, taking a bath, dressing, combing hair or using the toilet) while others reported their problematic ADLs more generic (e.g. problems with bathroom activities). Therefore, specific self-care ADLs like showering may perhaps even be underestimated.

\section{Occupational therapy}

COPD patients entering pulmonary rehabilitation experience clear difficulties with a variety of ADLs which cannot be explained confidently by clinical determinants. 
Therefore, an intake by an occupational therapist, including a semi-structured interview, like the COPM, seems necessary to develop a patient-tailored pulmonary rehabilitation program. Moreover, the current data clearly provide a rationale to assess the additional effects of occupational therapy on the functional performance of patients with COPD as part of a comprehensive pulmonary rehabilitation program. Indeed, energy conservation techniques in COPD patients during ADL reduces energy cost and dyspnea perception [35]. Reduced energy cost and dyspnea perception during $A D L$ may well increase performance and satisfaction scores of problematic ADLs. This warrants future randomized controlled trials.

To conclude, COPD patients have an enormous variety of problematic ADLs. Clinical determinants are weakly associated with problematic ADLs which emphasize the need of assessing problematic ADLs within the individual patient in order to plan tailored interventions.

\section{Acknowledgments}

Paul Willems of Human Movement Science, NUTRIM School for Nutrition, Toxicology and Metabolism, and Suzanne Goossens, Mieke Kersten, Michelle van Mook and Malou Lammers of the Department of Occupational Therapy, Zuyd University, Heerlen, Netherlands, are gratefully acknowledged for their help with electronic data processing. 


\section{References}

1. Young Y, Boyd CM, Guralnik JM, Fried LP (2010) Does self-reported function correspond to objective measures of functional impairment? J Am Med Dir Assoc 11: 645-653.

2. Nici L, Donner C, Wouters E, Zuwallack R, Ambrosino N, et al. (2006) American Thoracic Society/European Respiratory Society statement on pulmonary rehabilitation. Am J Respir Crit Care Med 173: $1390-1413$.

3. Stull DE, Leidy NK, Jones PW, Stahl E (2007) Measuring functional performance in patients with COPD: a discussion of patient-reported outcome measures. Curr Med Res Opin 23: 2655-2665.

4. Williams V, Bruton A, Ellis-Hill C, McPherson K (2007) What really matters to patients living with chronic obstructive pulmonary disease? An exploratory study. Chron Respir Dis 4: 77-85.

5. Leidy NK, Haase JE (1996) Functional performance in people with chronic obstructive pulmonary disease: a qualitative analysis. ANS Adv Nurs Sci 18: 77-89.

6. Law M, Baptiste S, Carswell A, McColl M, Polatajko H, et al. (1998) Canadian Occupational Performance Measure. Toronto: CAOT Publications ACE.

7. Sewell L, singh SJ (2001) The Canadian Occupational Performance Measure: is it a Reliable Measure in Clients with Chronic Obstructive Pulmonary Disease? Br J Occup Ther 64: 305-310.

8. Dedding C, Cardol M, Eyssen IC, Dekker J, Beelen A (2004) Validity of the Canadian Occupational Performance Measure: a client-centred outcome measurement. Clin Rehabil 18: 660-667.

9. Spruit MA, Vanderhoven-Augustin I, Janssen PP, Wouters EF (2008) Integration of pulmonary rehabilitation in COPD. Lancet 371: 12-13.

10. Sewell L, Singh SJ, Williams JE, Collier R, Morgan MD (2005) Can individualized rehabilitation improve functional independence in elderly patients with COPD? Chest 128: 1194-1200.

11. (2001) World Health Organization. International Classification of Functioning, Disability and Health: ICF. Geneva: World Health Organization.

12. Skumlien S, Haave E, Morland L, Bjortuft O, Ryg MS (2006) Gender differences in the performance of activities of daily living among patients with chronic obstructive pulmonary disease. Chron Respir Dis 3: 141-148.

13. Crockett AJ, Cranston JM, Moss JR, Alpers JH (2002) The impact of anxiety, depression and living alone in chronic obstructive pulmonary disease. Qual Life Res 11: 309-316.

14. Katz PP, Eisner MD, Yelin EH, Trupin L, Earnest G, et al. (2005) Functioning and psychological status among individuals with COPD. Qual Life Res 14: 1835-1843.

15. Tsukino M, Nishimura K, Ikeda A, Koyama H, Mishima M, et al. (1996) Physiologic factors that determine the health-related quality of life in patients with COPD. Chest 110: 896-903.

16. Katz PP, Gregorich S, Eisner M, Julian L, Chen H, et al. (2010) Disability in valued life activities among individuals with COPD and other respiratory conditions. J Cardiopulm Rehabil Prev 30: 126-136.

17. Okubadejo AA, O'Shea L, Jones PW, Wedzicha JA (1997) Home assessment of activities of daily living in patients with severe chronic obstructive pulmonary disease on long-term oxygen therapy. Eur Respir J 10: 1572-1575.

18. Garrod R, Bestall JC, Paul EA, Wedzicha JA, Jones PW (2000) Development and validation of a standardized measure of activity of daily living in patients with severe COPD: the London Chest Activity of Daily Living scale (LCADL). Respir Med 94: 589-596.

19. Probst VS, Troosters T, Coosemans I, Spruit MA, Pitta Fde O, et al. (2004) Mechanisms of improvement in exercise capacity using a rollator in patients with COPD. Chest 126: 1102-1107.

20. Mostert R, Goris A, Weling-Scheepers C, Wouters EF, Schols AM (2000) Tissue depletion and health related quality of life in patients with chronic obstructive pulmonary disease. Respir Med 94: 859867. 
21. den Hoed M, Westerterp KR (2008) Body composition is associated with physical activity in daily life as measured using a triaxial accelerometer in both men and women. Int J Obes (Lond) 32: 12641270.

22. Rodriguez Gonzalez-Moro JM, de Lucas Ramos P, Izquierdo Alonso JL, Lopez-Muniz Ballesteros B, Anton Diaz E, et al. (2009) Impact of COPD severity on physical disability and daily living activities: EDIP-EPOC I and EDIP-EPOC II studies. Int J Clin Pract 63: 742-750.

23. Jones PW (2007) Activity limitation and quality of life in COPD. Copd 4: 273-278.

24. (2002) ATS statement: guidelines for the six-minute walk test. Am J Respir Crit Care Med 166: 111117.

25. Troosters T, Gosselink R, Decramer M (1999) Six minute walking distance in healthy elderly subjects. Eur Respir J 14: 270-274.

26. Spruit MA, Wouters EF (2007) New modalities of pulmonary rehabilitation in patients with chronic obstructive pulmonary disease. Sports Med 37: 501-518.

27. Steiner MC, Barton RL, Singh SJ, Morgan MD (2002) Bedside methods versus dual energy X-ray absorptiometry for body composition measurement in COPD. Eur Respir J 19: 626-631.

28. Spruit MA, Pennings HJ, Janssen PP, Does JD, Scroyen S, et al. (2007) Extra-pulmonary features in COPD patients entering rehabilitation after stratification for MRC dyspnea grade. Respir Med 101: 2454-2463.

29. Lexell EM, Iwarsson S, Lexell J (2006) The complexity of daily occupations in multiple sclerosis. Scand J Occup Ther 13: 241-248.

30. Kjeken I, Slatkowsky-Christensen B, Kvien TK, Uhlig T (2004) Norwegian version of the Canadian Occupational Performance Measure in patients with hand osteoarthritis: validity, responsiveness, and feasibility. Arthritis Rheum 51: 709-715.

31. Law M, Baptiste S, McColl M, Opzoomer A, Polatajko H, et al. (1990) The Canadian occupational performance measure: an outcome measure for occupational therapy. Can J Occup Ther 57: 82-87.

32. Lacasse Y, Brosseau L, Milne S, Martin S, Wong E, et al. (2002) Pulmonary rehabilitation for chronic obstructive pulmonary disease. Cochrane Database Syst Rev: CD003793.

33. Nicolson $P$, Anderson $P$ (2003) Quality of life, distress and self-esteem: a focus group study of people with chronic bronchitis. Br J Health Psychol 8: 251-270.

34. Singh S (ERS congress, 2008) Out-Patient Pulmonary Rehabilitation. http://www.erseducation.org/pages/default. aspx?id=335. Pisa.

35. Velloso M, Jardim JR (2006) Study of energy expenditure during activities of daily living using and not using body position recommended by energy conservation techniques in patients with COPD. Chest 130: 126-132. 



\section{Chapter 3}

Objective physical activity assessment in patients with chronic organ failure: a validation study of a new single-unit activity monitor

Janneke Annegarn, Martijn A. Spruit, Nicole H.M.K. Uszko-Lencer, Sophie Vanbelle, Hans H.C.M. Savelberg, Annemie M.W.J. Schols, Emiel F.M. Wouters and Kenneth Meijer.

Arch Phys Med Rehabil, 2011. 92: 1852-1857 e1851 


\begin{abstract}
The aim of this study was to validate a new activity monitor (CAM) in patients with chronic organ failure during one hour of unconstrained activity assessment. In this validation study, participants wore a CAM (placed on the thigh) for one hour while they were video taped. Participants were instructed to continue their normal daily routine at the rehabilitation centre $\mathrm{CIRO}+$, a centre of expertise for chronic organ failure. Ten chronic organ failure patients participated with a large range of functional exercise capacity. Cohen's kappa coefficients for transient events resulted in an almost perfect agreement $(0.85 \pm 0.08)$ between time spend on activities and postures according to video and CAM. The CAM is a promising single-sensor unobtrusive tool for providing accurate data on the type and duration of daily activities in the home environment of patients with chronic organ failure.
\end{abstract}




\section{Introduction}

Patients with chronic organ failure, like chronic heart failure (CHF) or chronic obstructive pulmonary disease (COPD) are generally characterized by an inactive lifestyle in order to avoid the unpleasant sensation of dyspnea [1,2]. Indeed, walking is the number one problematic activity of daily life [3]. Decreased weight bearing activities and postures in daily life (e.g. walking and standing, respectively) are most probably important triggers in the development and/or progression of lower-limb muscle atrophy, muscle weakness and exercise intolerance in patients with CHF or COPD $[4,5,6]$. Indeed, a "downward disease spiral" has been postulated, in which advancing dyspnea leads to a sedentary lifestyle and de-conditioning of the lowerlimb muscles, and thus further daily physical inactivity [7]. Moreover, exercisebased cardiopulmonary rehabilitation programs have been shown to effectively improve quadriceps muscle mass, lower-limb muscle function and exercise capacity in patients with CHF or COPD $[8,9]$.

To date, daily physical activity levels have not yet been implemented as outcome measure of cardiopulmonary rehabilitation $[10,11]$. Indeed, more traditional measures of exercise performance, such as the six minute walk test, are assumed to reflect daily physical activity levels in patients with CHF or COPD $[1,6,12]$. However, an increased exercise capacity after cardiopulmonary rehabilitation does not necessarily result in a more active lifestyle $[13,14]$. Therefore, it seems reasonable to add the objective assessment of daily physical activity levels to the outcomes assessed before and after cardiopulmonary rehabilitation [15]. Furthermore, objective assessment of daily physical activity levels may also be useful as a coaching tool to maintain and/or enhance a more physically active lifestyle [16].

Questionnaires show limited validity and reliability to objectively assess daily physical activity levels [17], while wearable devices gain in popularity as a method to objectify daily life activity $[1,6,18,19,20,21,22,23,24]$. Unfortunately, most commercially available devices that aim to assess physical activity in CHF and/or COPD patients have technical and/or methodological limitations. For example, the Dynaport Activity Monitor accurately reflects time spend in different postures and activities in COPD patients during a standardized protocol [17]. However, it consists of multiple units and, in turn, will be more obtrusive for patients compared to single-unit devices. Moreover, the Dynaport Activity Monitor has never been validated during 'free living' conditions in which subjects follow their normal routine (e.g. not restricted to perform specific activities); and a drop out rate of $19 \%$ of the subjects has been reported for two days of activity assessment due to misplacement or 
technical problems [25]. The SenseWear is a single-unit activity monitor that does not provide information on time spent in postures and the amounts of steps counted by the device are inaccurate [26]. The Minimod is also a single-unit device that does provide accurate information on time spent in different postures and activities [26]. However, this device seems to become less accurate in patients that walk slowly and has also never been validated during 'free living' conditions [26]. Therefore, the aim of this study was to validate a new single-unit activity monitor in both healthy subjects during a standardized protocol and in CHF or COPD patients with a wide range in functional exercise capacity during 'free living' conditions.

\section{Methods}

\section{Design and participants}

Two studies were designed to validate a new single-unit activity monitor (CAM). Study A evaluated the accuracy of the activity monitor using a standardized proto$\mathrm{col}$ in a group of five healthy subjects (all students; 3 men; age: $23.8 \pm 3.6$ years; body mass index (BMI): $21.0 \pm 2.0 \mathrm{~kg} / \mathrm{m}^{2}$ ). Study B evaluated the accuracy of the activity monitor during one hour of unconstrained activity and posture assessment in five patients with CHF (4 men; age: $54.2 \pm 16.8$ years; LVEF: $32.4 \pm 7.6 \%$; NYHA class I, $\mathrm{n}=1$; NYHA class II, $\mathrm{n}=2$ and NYHA class III, $\mathrm{n}=2 ; \mathrm{BMI}: 30.4 \pm 6.9 \mathrm{~kg} / \mathrm{m}^{2}$ ) and five patients with COPD ( 2 men; age: $60.0 \pm 9.9$ years; $\mathrm{FEV}_{1}$ : $51.6 \pm 36.2 \%$ of predicted value; GOLD stage I, $n=1$; GOLD stage II, $n=1$; GOLD stage III, $n=1$; and GOLD stage IV, $n=2$; BMI: $19.7 \pm 1.9 \mathrm{~kg} / \mathrm{m}^{2}$ ). All patients were clinically stable. In both studies the results of 2 attachment sides of the CAM were compared: on the frontal part of the thigh just above the knee $\left(\mathrm{CAM}_{\text {leg }}\right)$ and on the lower back at the height of the second lumbar vertebra, nearby the body's centre of mass $\left(C A M_{\text {trunk }}\right)$. The current protocol was approved by the Medical Ethical Committee of Maastricht University Medical Centre+ and conformed to the principles outlined in the World Medical Association declaration of Helsinki which have been revised in Seoul (approval number: NL30747.068.09).

\section{New single-unit activity monitor}

Recently, a new single-unit activity monitor was developed by Maastricht Instruments B.V. (Maastricht, the Netherlands): the CAM. It contains a triaxial piezoresistive accelerometer, weighs 102 gram and has a range of \pm 4 times gravity (G) with a resolution of $0.02 \mathrm{G}$. The measured signal is stored on a $2 \mathrm{~GB}$ micro SD cart at a sample rate of 102 Hertz. The CAM classifies activities and/or postures using a windowing technique in which a threshold on several accelerometer features is applied. The basic methodology is described in more detail by Preece et al [27]. A hierarchical classification scheme is used (figure 3.1), which first differentiates between 
dynamic activities and static postures using a parameter for intensity of motion: the signal magnitude area (SMA). Subsequently static postures are differentiated into weight bearing and non-weight bearing postures using a parameter for orientation of the CAM: the mean accelerometer signal of the respective axis (MI).

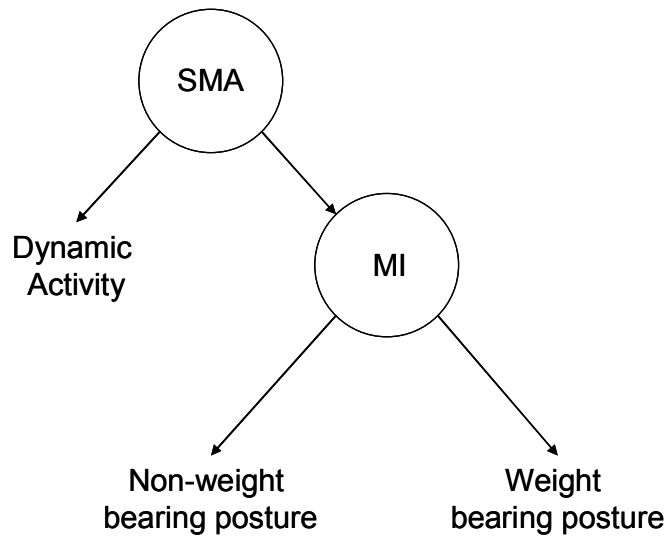

Figure 3.1. This classification scheme separates dynamic activities from postures and within the postures non-weight bearing postures and weight bearing postures from each other by means of two parameters. The first parameter differentiates between dynamic activity and static postures using the signal magnitude area (SMA), a parameter for intensity of motion. The second parameter differentiates between nonweight bearing postures and weight bearing postures using the mean accelerometer signal of the respective axis (MI), a parameter for orientation of the CAM.

Study A: A group of five healthy subjects underwent a standardized protocol at the Department of Human Movement Sciences of the Maastricht University: standing upright (120sec), sitting upright (120sec), supine position (120sec), walking at a selfselected speed $(120 \mathrm{sec})$, and unloaded cycling at a self-selected number of revolutions per minute $(120 \mathrm{sec})$. Between different activities and/or postures, subjects had 30 seconds transition time. These transitions were excluded from further analyses. The accuracy of the CAM leg $_{\text {end }}$ CAM trunk (worn simultaneously) was calculated and expressed as the percentage of correctly classified seconds. In addition, we investigated whether the $\mathrm{CAM}_{\text {leg }}$ and $\mathrm{CAM}_{\text {trunk }}$ could accurately reflect subject's movement intensity. For this purpose, all subjects walked on a treadmill at five different walking speeds $(1.5,2.5,3.5,4.5,5.5$ and $6.5 \mathrm{~km} / \mathrm{h})$. Subjects walked each speed for 120 seconds with 30 seconds transition time in between.

Study B: Five patients with CHF and five patients with COPD were recruited during their rehabilitation program at $\mathrm{CIRO}+$, a centre of expertise for chronic organ failure in Horn, the Netherlands. All patients were free of disease-related hospitalizations for at least 4 weeks. The 6-minute walk test (6MWT) was performed as a measure of functional exercise capacity in accordance with the guidelines of the American 
Thoracic Society [28], including a practice walk. Moreover, all patients wore the $\mathrm{CAM}_{\text {leg }}$ and the $\mathrm{CAM}_{\text {trunk }}$ simultaneously for at least one hour and were instructed to continue their normal daily routine. After wearing the CAM devices, patients estimated the percentage of time spent on non-weight bearing postures (e.g., sitting or lying), weight bearing postures (e.g., standing) and dynamic activities (e.g., walking). Video recordings were used as the criterion standard $[17,29]$. Patients were videotaped by the researcher while they wore the $C A M_{\text {leg }}$ and the $C A M_{\text {trunk }}$. If the patient decided go somewhere (lunch, appointment, etc.), the researcher followed the patient with the camera. After the recordings, the tapes were analyzed by one examiner, who was blinded to the values obtained by the CAMs and patients' estimation. In addition, a video recording of one subject was classified by another researcher to establish the inter-examiner reliability and to ensure there was no examiner bias in analyzing the video recordings [30]. A second by second analyses showed an overall agreement of $99.8 \%$ between two examiners.

\section{Statistical Analysis}

Results were expressed as mean \pm standard deviation (SD) for quantitative variables, while frequencies and proportions were used for categorical variables. The association between walking speeds and the movement intensity parameter SMA was analyzed by means of the generalized linear mixed models (GLMM) approach, accounting for repeated data within each subject. The accuracy of the CAM $\mathrm{M}_{\text {leg }}$ and $\mathrm{CAM}_{\text {trunk }}$ during the standardized protocol was defined as the proportion of seconds correctly classified postures and activities with respect to the actual performed postures and activities as defined in the protocol.

The time spent in weight bearing postures, non-weight bearing postures and dynamic activities was determined for each subject using video recordings, $\mathrm{CAM}_{\text {leg, }}$ $\mathrm{CAM}_{\text {trunk }}$ and patients' estimation. If the examiner could not identify patients' posture or activity (e.g. due to toilet visit), the seconds (samples) were removed from further analyses. Since the subjects were not all observed during exactly $3600 \mathrm{sec}-$ onds (mean duration: $3672 \mathrm{sec}$, range: $3416-3815 \mathrm{sec}$ ), the time spent in the different postures and activities were recalculated on a standard period of 3600 seconds. Mean differences between video recordings, $\mathrm{CAM}_{\text {leg, }} \mathrm{CAM}_{\text {trunk }}$ and patients' estimation were studied using GLMM to account for repeated measures within subjects. Bland and Altman plots [31] were displayed to depict disagreements between video

recordings and $\mathrm{CAM}_{\text {leg, }} \mathrm{CAM}_{\text {trunk }}$ and patients' estimation with respect to the time spent in weight bearing postures, non-weight bearing postures and dynamic activities. 
Weight bearing postures, non-weight bearing postures and dynamic activities were classified each second using video recordings, $C A M_{\text {leg }}$ and $C A M_{\text {trunk }}$. The agreement between the classification made by the video recording and $C A M_{\text {leg }}$ and $C A M_{\text {trunk }}$ was determined for each subject using Cohen's kappa coefficient for transient events [32]. This agreement index takes into account possible serial correlation existing among observations made on the same subject while being interpreted in the same way than the classical Cohen's kappa coefficient [33].

Results were considered to be significant at the $5 \%$ critical level $(p<0.05)$. All statistical calculations were performed using SAS (version 9.2 for Windows) and R (version 2.12.1) statistical package. 


\section{Results}

Study A: All subjects completed the standardized protocol. Dynamic activities (walking and cycling), weight bearing (standing) and non-weight bearing (sitting, lying) postures were all correctly detected by the $\mathrm{CAM}_{\text {leg }}\left(100 \%\right.$ accuracy). The $\mathrm{CAM}_{\text {trunk }}$ also correctly detected all activities, except cycling ( $95 \%$ accuracy). The movement intensity parameter of the CAM $\mathrm{CAg}_{\text {leg }}(p<0.001)$ and $\mathrm{CAM}_{\text {trunk }}(p<0.001)$ was closely related to the treadmill walking speed (figure 3.2).

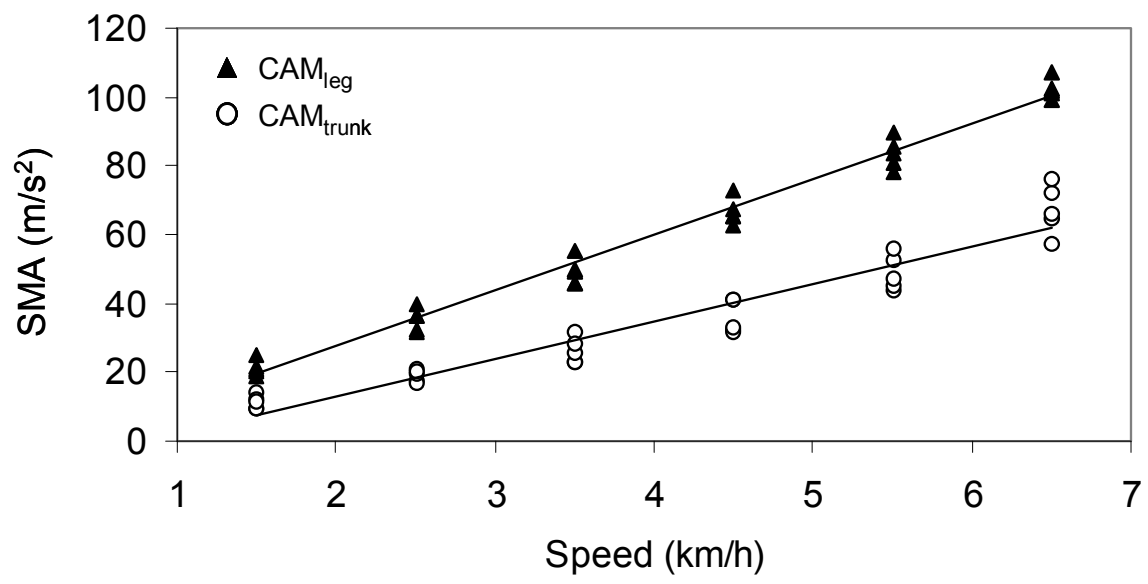

Figure 3.2: The relation between the movement intensity parameter (SMA) of the $C A M_{\text {leg }}$ and $C A M_{\text {trunk }}$ and treadmill walking speed.

Study B: Patients showed a large range in functional exercise capacity as measured by the 6MWT (mean distance: $493 \mathrm{~m}$, range: $204-755 \mathrm{~m}$ ). Patients performed \pm 1 hour of self-chosen activities and postures, like walking, standing and sitting. None of the patients spent time lying. All patients completed the one-hour measurement. According to video analyses the subjects spent on average $2590 \pm 672$ seconds in non-weight bearing postures, $263 \pm 182$ seconds in weight bearing postures and $747 \pm 506$ seconds in dynamic activities.

The estimated time spent in weight bearing postures, non-weight bearing postures and dynamic activities differed significantly between the $C A M_{\text {trunk }}$ and the video recording $(p<0.01)$ and between the $C A M_{\text {leg }}$ and the $C A M_{\text {trunk }}(p<0.01)$ (figure 3.3). The $\mathrm{CAM}_{\text {trunk }}$ overestimated the time spent in weight bearing postures and underestimated the time spent in non-weight bearing postures. The $\mathrm{CAM}_{\text {trunk }}$ results also 
slightly differed from the patients' estimation $(p<0.05)$ for weight-bearing postures. Finally, the $\mathrm{CAM}_{\text {leg }}$ overestimated the time spent in weight bearing postures with respect to the video recording $(p<0.01)$. Note that the accuracy of the $C A M_{\text {trunk }}$ with video recording was $67.7 \%$, while $\mathrm{CAM}_{\text {leg }}$ and the patients' estimation reached an accuracy of $94.4 \%$ and $83.0 \%$, respectively.

Results were confirmed in the Bland and Altman plots. No constant bias was observed between the $\mathrm{CAM}_{\text {leg }}$ and the video for the three different postures. The same holds between patients' estimation and the video, with the difference that the results showed a higher variability than with the CAM leg. Finally, $\mathrm{CAM}_{\text {trunk }}$ showed disagreement with video. The longer the time spent in the weight bearing posture, the higher the bias with the video (figure 3.4). 
weight bearing postures

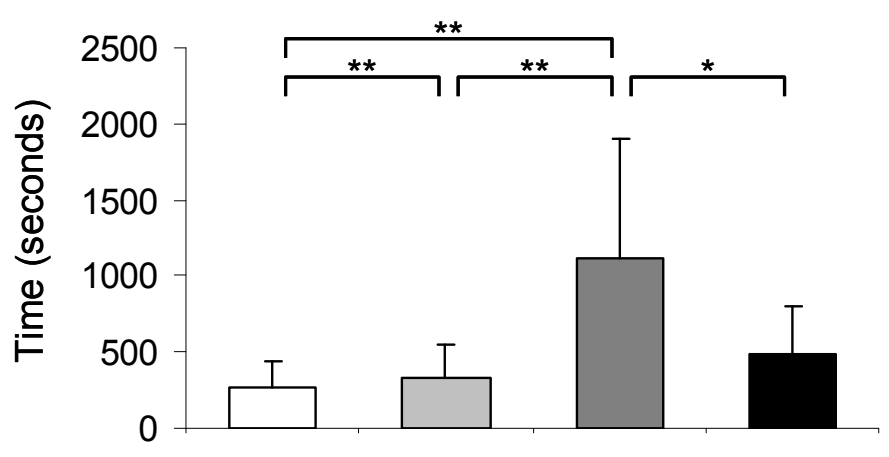

non-weight bearing postures

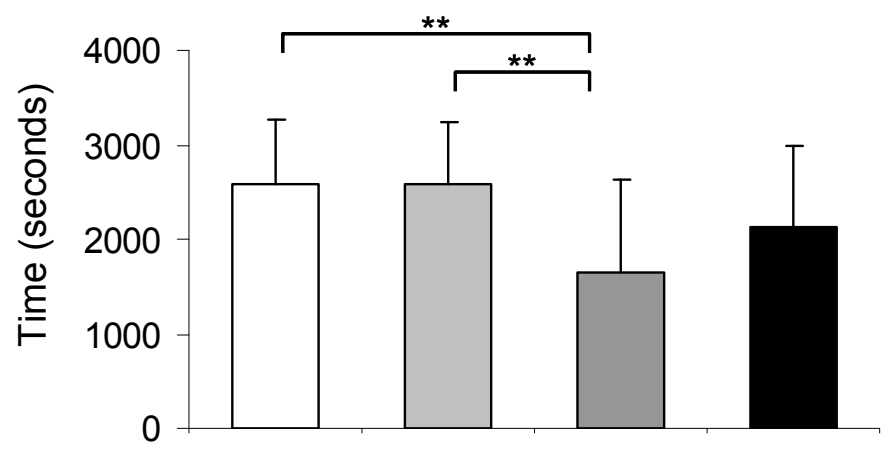

dynamic activity

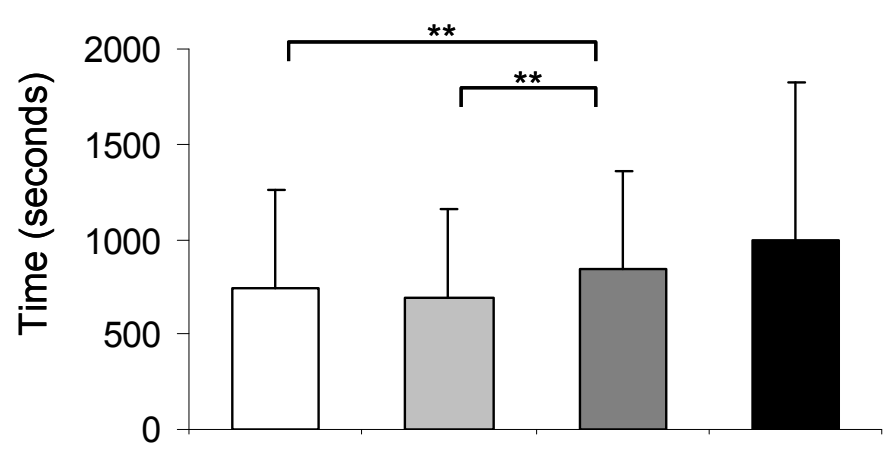

Figure 3.3: Time spent on different activities obtained by video analysis (white), CAM $_{\text {leg }}$ (light grey), $\mathrm{CAM}_{\text {trunk }}$ (dark grey) and patients' estimation (black). ${ }^{*}: \mathrm{p}<0.05, * *<0.01$ 

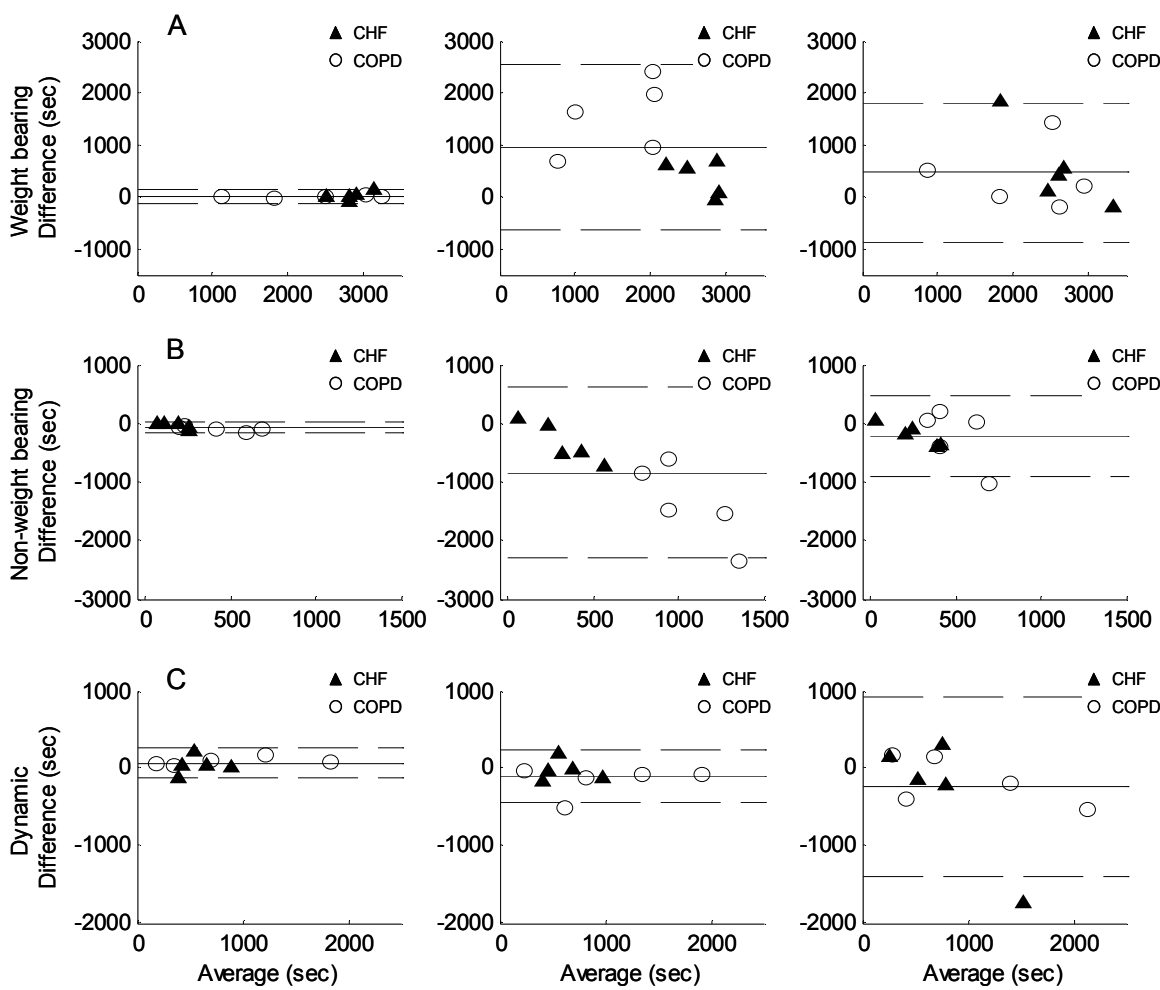

Figure 3.4: Bland and Altman plots for comparison of $(A)$ weight bearing postures, $(B)$ non-weight bearing postures, and (C) dynamic activity assessed by video recordings (criterion standard), $C_{\text {leg, }} \mathrm{CAM}_{\text {trunk }}$ and

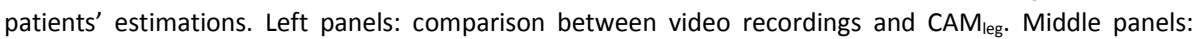
comparison between video recordings and $\mathrm{CAM}_{\text {trunk. }}$ Right panels: comparison between video recordings and patients' estimations. Solid lines represent the mean difference and the dotted lines represent the mean difference \pm 1.96 standard deviation of the difference. 
For all subjects, the $\mathrm{CAM}_{\text {leg }}$ and video recordings presented substantial to almost perfect agreement in the classification of seconds in weight bearing, non-weight bearing and dynamic activities. Agreement, using Cohen's kappa coefficient for transient events, was on average $0.85 \pm 0.08$. The minimum value of 0.70 was observed for subject 4 and the maximum value of 0.95 for subject 3 . The agreement between video recordings and $\mathrm{CAM}_{\text {trunk }}$ was substantially lower. The agreement coefficients varied between 0.04 and 0.87 and were on average $0.47 \pm 0.29$ (table 3.1).

Table 3.1: Cohen's kappa index for each patient

\begin{tabular}{|c|c|c|c|}
\hline Group & Patient & $\begin{array}{c}\text { Kappa CAM leg } \\
(95 \% \mathrm{Cl})\end{array}$ & $\begin{array}{c}\text { Kappa CAM } \text { trunk } \\
(95 \% \mathrm{Cl})\end{array}$ \\
\hline & 1 & $0.85(0.80-0.87)$ & $0.34(0.23-0.40)$ \\
\hline & 2 & $0.73(0.64-0.76)$ & $0.08(0.02-0.13)$ \\
\hline \multirow[t]{5}{*}{$\mathrm{CHF}$} & 3 & $0.95(0.90-0.96)$ & $0.65(0.58-0.70)$ \\
\hline & 4 & $0.70(0.49-0.76)$ & $0.44(0.33-0.53)$ \\
\hline & 5 & $0.94(0.84-0.95)$ & $0.04(0.00-0.09)$ \\
\hline & 6 & $0.90(0.83-0.93)$ & $0.84(0.80-0.87)$ \\
\hline & 7 & $0.85(0.82-0.87)$ & $0.40(0.30-0.46)$ \\
\hline \multirow[t]{3}{*}{ COPD } & 8 & $0.91(0.85-0.93)$ & $0.87(0.72-0.90)$ \\
\hline & 9 & $0.86(0.80-0.89)$ & $0.38(0.22-0.50)$ \\
\hline & 10 & $0.80(0.68-0.85)$ & $0.65(0.37-0.72)$ \\
\hline
\end{tabular}

For all kappa values $\mathrm{P}<0.001$ 


\section{Discussion}

The aim of this study was to validate a new single-unit activity monitor in both healthy subjects and in patients with moderate to very severe CHF or COPD. The results of our study show that the CAM worn on the upper leg is an accurate method for measuring the time spent in weight bearing postures, non-weight bearing postures and dynamic activities in healthy subjects during a standardized protocol and in patients with CHF or COPD during 'free living' conditions.

Although the CAM worn at the trunk was able to accurately assess dynamic activities in healthy subjects under standardized conditions, low agreements were obtained between video analysis and the $\mathrm{CAM}_{\text {trunk }}$ for weight bearing and non-weight bearing postures in patients with CHF and COPD during 'free living' conditions. Furthermore, patients' estimation of time spent on activities of only one hour show an important inaccuracy compared with video recordings, which was also found by Pitta et al. [17].

Compared to data reported for the SenseWear [26], which cannot differentiate between sitting and standing, the CAM is superior in its ability to detect time spent in dynamic activity and time spent in different postures. The accuracy of the CAM leg in detecting weight bearing postures, non-weight bearing postures and dynamic activities is comparable to the accuracy of the Dynaport Activity Monitor as validated in COPD patients [17] and an activity monitor validated in CHF patients [2]. The main advantage of the CAM is that it consists of a single unit, while the Dynaport Activity Monitor and AM consist of multiple units worn on different body parts connected by wires. In fact, Pitta et al. reported a drop out of $19 \%$ of the subjects using the Dynaport Activity Monitor due to misplacement of the device and technical problems [25], while Van den Berg-Emons et al. reported that the AM was experienced by some patients as being rather heavy and one patient with severe skin problems did not tolerated the materials [2]. In the current validation study, patients had no complaints while wearing the CAM. Future research is recommended to assess the user friendliness (e.g. wearing comfort, attachment difficulty, etc.) of the CAM and other activity monitors while wearing the devices for longer periods of time.

Another single-unit device validated in COPD patients worn on the lower back is the Minimod [26]. This device appears to correctly identify different movements and postures during a standardized protocol with an accuracy of 98.7\% [26]. However, this does not necessarily mean that similar accuracy is guaranteed under free living

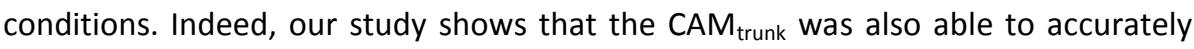
identify different postures and dynamic activity under standardized conditions (98.9\%), but showed low agreement with video recordings during unconstrained 
conditions (67.7\%). Misclassification mainly occurred when COPD patients leaned forward while sitting, a typical behavior for COPD patients since it increases their ventilatory capacity [34]. This forward sitting posture was classified by the CAM trunk $_{\text {. }}$ as standing, because gravitational accelerations in the anterior-posterior signal were in the range of the posture standing.

\section{Limitations and Future Perspectives}

This study is limited by the fact that patients are not measured in their own environment (i.e. at home). This may affect the generalizability of the results toward activities performed in daily life. However, the approach was effective as patients showed in current 'free living' conditions behavior (sitting while leaning to the front) leading to misclassification of the $\mathrm{CAM}_{\text {trunk }}$ that would most likely not have appeared during a standardized protocol in which patients are told how to sit on a specific chair. In contrast to the CAM trunk, the CAM leg is less likely to overlap in orientation during sitting and standing. On the other hand, the orientation of the $\mathrm{CAM}_{\text {leg }}$ is similar during sitting and lying, while this is less likely in the CAM trunk. Because lying and sitting are both non-weight bearing postures, they require similar amount of energy [35]. Therefore it is preferable to wear the CAM on the thigh, allowing a more robust classification in unconstrained conditions between weight bearing and non-weight bearing postures.

Similar to other activity monitors worn on the trunk and/or leg $[17,26]$, the CAM does not provide information on upper-limb activities during postures and walking. For more specific upper-limb activity assessment, it is preferable to wear an activity monitor on the arm.

The validity of the CAM was tested in a relatively small patient population $(n=10)$ with a wide range in functional exercise capacity. Although the number of samples used to perform the validation in each subject was high $(n=3600)$, an evaluation of the CAM in a greater population would be useful.

Spending too much time in non-weight bearing postures is an important factor in the development of skeletal muscle mass depletion and dysfunction (loss of strength and/or endurance) $[4,5,6]$. These features have been associated with exercise intolerance $[25,36]$, increased hospitalization [37,38], an overall loss of health status [39] and reduced survival [40]. Therefore it is important to assess dynamic activity and to differentiate between time spend on non-weight bearing postures and time spend on weight bearing postures. These assessments could provide a prognostic value of physical activity and thus serve as an important outcome measure of cardiopulmonary rehabilitation. Possibly these assessments will also be useful as a coaching tool to maintain and/or enhance a more physical active lifestyle in patients with CHF or COPD. 
To conclude, the $\mathrm{CAM}_{\text {leg }}$ accurately assessed the time spent in weight bearing postures, non-weight bearing postures and dynamic activity in patients with CHF and COPD in 'free-living' conditions. The $\mathrm{CAM}_{\text {trunk }}$ resulted in large disagreement with the video recordings for identifying postures. Patients' estimations resulted in large disagreement with video recordings for all activities and postures. Therefore, we conclude that the $\mathrm{CAM}_{\text {leg }}$ is a promising unobtrusive tool for providing accurate data on the type and duration of daily physical activity and postures in the home environment of CHF and COPD patients. 


\section{References}

1. Pitta F, Troosters T, Probst VS, Spruit MA, Decramer M, et al. (2006) Quantifying physical activity in daily life with questionnaires and motion sensors in COPD. Eur Respir J 27: 1040-1055.

2. van den Berg-Emons HJ, Bussmann JB, Balk AH, Stam HJ (2000) Validity of ambulatory accelerometry to quantify physical activity in heart failure. Scand J Rehabil Med 32: 187-192.

3. Annegarn J, Meijer K, Passos VL, Stute K, Wiechert J, et al. (2012) Problematic activities of daily life are weakly associated with clinical characteristics in COPD. J Am Med Dir Assoc 13: 284-290.

4. Gosker HR, Lencer NH, Franssen FM, van der Vusse GJ, Wouters EF, et al. (2003) Striking similarities in systemic factors contributing to decreased exercise capacity in patients with severe chronic heart failure or COPD. Chest 123: 1416-1424.

5. Spruit MA, Gosselink R, Troosters T, Kasran A, Gayan-Ramirez G, et al. (2003) Muscle force during an acute exacerbation in hospitalised patients with COPD and its relationship with CXCL8 and IGF-I. Thorax 58: 752-756.

6. Jehn M, Schmidt-Trucksass A, Schuster T, Weis M, Hanssen H, et al. (2009) Daily walking performance as an independent predictor of advanced heart failure: Prediction of exercise capacity in chronic heart failure. Am Heart J 157: 292-298.

7. Polkey MI, Moxham J (2006) Attacking the disease spiral in chronic obstructive pulmonary disease. Clin Med 6: 190-196.

8. Spruit MA, Wouters EF (2007) New modalities of pulmonary rehabilitation in patients with chronic obstructive pulmonary disease. Sports Med 37: 501-518.

9. Adamopoulos S, Parissis JT, Kremastinos DT (2003) New aspects for the role of physical training in the management of patients with chronic heart failure. Int J Cardiol 90: 1-14.

10. Lacasse Y, Brosseau L, Milne S, Martin S, Wong E, et al. (2002) Pulmonary rehabilitation for chronic obstructive pulmonary disease. Cochrane Database Syst Rev: CD003793.

11. Davies EJ, Moxham T, Rees K, Singh S, Coats AJ, et al. (2010) Exercise based rehabilitation for heart failure. Cochrane Database Syst Rev: CD003331.

12. Walker PP, Burnett A, Flavahan PW, Calverley PM (2008) Lower limb activity and its determinants in COPD. Thorax 63: 683-689.

13. Pitta F, Troosters T, Probst VS, Langer D, Decramer M, et al. (2008) Are Patients with Copd More Active after Pulmonary Rehabilitation ? Chest 134: 273-280.

14. van den Berg-Emons R, Balk A, Bussmann H, Stam H (2004) Does aerobic training lead to a more active lifestyle and improved quality of life in patients with chronic heart failure? Eur $\mathrm{J}$ Heart Fail 6: 95-100.

15. Spruit MA, Vanderhoven-Augustin I, Janssen PP, Wouters EF (2008) Integration of pulmonary rehabilitation in COPD. Lancet 371: 12-13.

16. Benzo R (2009) Activity monitoring in chronic obstructive pulmonary disease. J Cardiopulm Rehabil Prev 29: 341-347.

17. Pitta F, Troosters T, Spruit MA, Decramer M, Gosselink R (2005) Activity monitoring for assessment of physical activities in daily life in patients with chronic obstructive pulmonary disease. Arch Phys Med Rehabil 86: 1979-1985.

18. Furlanetto KC, Bisca GW, Oldemberg N, Sant'anna TJ, Morakami FK, et al. (2010) Step counting and energy expenditure estimation in patients with chronic obstructive pulmonary disease and healthy elderly: accuracy of 2 motion sensors. Arch Phys Med Rehabil 91: 261-267.

19. van den Berg-Emons RJ, Bussmann JB, Balk AH, Stam HJ (2005) Factors associated with the level of movement-related everyday activity and quality of life in people with chronic heart failure. Phys Ther 85: 1340-1348. 
20. Grant PM, Ryan CG, Tigbe WW, Granat MH (2006) The validation of a novel activity monitor in the measurement of posture and motion during everyday activities. Br J Sports Med 40: 992-997.

21. Hecht A, Ma S, Porszasz J, Casaburi R (2009) Methodology for using long-term accelerometry monitoring to describe daily activity patterns in COPD. Copd 6: 121-129.

22. Pitta F, Takaki MY, Oliveira NH, Sant'anna TJ, Fontana AD, et al. (2008) Relationship between pulmonary function and physical activity in daily life in patients with COPD. Respir Med 102: 1203-1207.

23. Troosters T, Sciurba F, Battaglia S, Langer D, Valluri SR, et al. (2010) Physical inactivity in patients with COPD, a controlled multi-center pilot-study. Respir Med 104: 1005-1011.

24. Cohen MD, Cutaia M (2010) A Novel Approach to Measuring Activity in Chronic Obstructive Pulmonary Disease: using 2 activity monitors to classify daily activity. J Cardiopulm Rehabil Prev 30: 186194.

25. Pitta F, Troosters T, Spruit MA, Probst VS, Decramer M, et al. (2005) Characteristics of physical activities in daily life in chronic obstructive pulmonary disease. Am J Respir Crit Care Med 171: 972 977.

26. Langer D, Gosselink R, Sena R, Burtin C, Decramer M, et al. (2009) Validation of two activity monitors in patients with COPD. Thorax 64: 641-642.

27. Preece SJ, Goulermas JY, Kenney LPJ, Howard D, Meijer K, et al. (2009) Activity identification using body-mounted sensors-a review of classification techniques. Physiological Measurement 30: R1R33.

28. (2002) ATS statement: guidelines for the six-minute walk test. Am J Respir Crit Care Med 166: 111117.

29. Uiterwaal M, Glerum EB, Busser HJ, van Lummel RC (1998) Ambulatory monitoring of physical activity in working situations, a validation study. J Med Eng Technol 22: 168-172.

30. Bussmann JB, Tulen JH, van Herel EC, Stam HJ (1998) Quantification of physical activities by means of ambulatory accelerometry: a validation study. Psychophysiology 35: 488-496.

31. Bland JM, Altman DG (1986) Statistical methods for assessing agreement between two methods of clinical measurement. Lancet 1: 307-310.

32. Norman RG, Scott MA (2007) Measurement of inter-rater agreement for transient events using Monte Carlo sampled permutations. Stat Med 26: 931-942.

33. Landis JR, Koch GG (1977) The measurement of observer agreement for categorical data. Biometrics 33: 159-174.

34. Probst VS, Troosters T, Coosemans I, Spruit MA, Pitta Fde O, et al. (2004) Mechanisms of improvement in exercise capacity using a rollator in patients with COPD. Chest 126: 1102-1107.

35. (1985) Energy and protein requirements. Report of a joint FAO/WHO/UNU Expert Consultation. World Health Organ Tech Rep Ser 724: 1-206.

36. Gosselink R, Troosters T, Decramer M (1996) Peripheral muscle weakness contributes to exercise limitation in COPD. Am J Respir Crit Care Med 153: 976-980.

37. Decramer M, Gosselink R, Troosters T, Verschueren M, Evers G (1997) Muscle weakness is related to utilization of health care resources in COPD patients. Eur Respir J 10: 417-423.

38. Garcia-Aymerich J, Farrero E, Felez MA, Izquierdo J, Marrades RM, et al. (2003) Risk factors of readmission to hospital for a COPD exacerbation: a prospective study. Thorax 58: 100-105.

39. Mostert R, Goris A, Weling-Scheepers C, Wouters EF, Schols AM (2000) Tissue depletion and health related quality of life in patients with chronic obstructive pulmonary disease. Respir Med 94: 859867.

40. Schols AM, Broekhuizen R, Weling-Scheepers CA, Wouters EF (2005) Body composition and mortality in chronic obstructive pulmonary disease. Am J Clin Nutr 82: 53-59. 



\section{Chapter 4}

\section{Differences in walking pattern during 6-min walk test between patients with COPD and healthy subjects}

Janneke Annegarn, Martijn A. Spruit, Hans H.C.M. Savelberg, Paul J.B. Willems, Coby van de Bool, Annemie M.W.J. Schols, Emiel F.M. Wouters and Kenneth Meijer.

PLoS One 7: e37329 (2012) 


\begin{abstract}
To date, detailed analyses of walking patterns using accelerometers during the 6min walk test (6MWT) have not been performed in patients with chronic obstructive pulmonary disease (COPD). Therefore, it remains unclear whether and to what extent COPD patients have an altered walking pattern during the 6MWT compared to healthy elderly subjects. 79 COPD patients and 24 healthy elderly subjects performed the 6MWT wearing an accelerometer attached to the trunk. The accelerometer features (walking intensity, cadence, and walking variability) and subject characteristics were assessed and compared between groups. Moreover, associations were sought with 6-min walk distance (6MWD) using multiple ordinary least squares (OLS) regression models. COPD patients walked with a significantly lower walking intensity, lower cadence and increased walking variability compared to healthy subjects. Walking intensity and height were the only two significant determinants of 6MWD in healthy subjects, explaining $85 \%$ of the variance in 6MWD. In COPD patients also age, cadence, walking variability measures and their interactions were included were significant determinants of 6MWD (total variance in 6MWD explained: $88 \%$ ). COPD patients have an altered walking pattern during 6MWT compared to healthy subjects. These differences in walking pattern partially explain the lower 6MWD in patients with COPD.
\end{abstract}




\section{Introduction}

The 6-minute walk test (6MWT) is commonly used to assess functional exercise performance in patients with chronic obstructive pulmonary disease (COPD) [1]. It is a practical, relatively simple test which has gained importance in evaluating the functional status of patients with COPD [2]. Moreover, a poor 6-minute walk distance (6MWD, <350 meters) has prognostic value in patients with COPD [2].

The 6MWD cannot be confidently predicted from conventional descriptors of COPD, such as the Global Initiative for Chronic Obstructive Lung Disease (GOLD) stage or the Medical Research Council (MRC) scale [3]. Therefore, it is necessary to assess functional exercise performance in daily clinical practice in patients with COPD.

Walking patterns are generally influenced by the trade-off between the requirements to minimize energetic costs and to maintain stability [4]. Indeed, walking is particularly unstable in the medio-lateral direction (Figure 4.1). To compensate for balance disturbances during walking, active adjustment of the step width (largely due to through lateral foot placement) is necessary resulting in variability in walking pattern [4]. Then again, to reduce energetic costs of walking, variability in walking pattern needs to be minimized [5]. In COPD patients, different clinical characteristics, such as decreased lower-limb muscle function [6] and a disturbed balance [7], may compromise the ability to balance the energetic and stability requirements posed by walking. Hence, the walking pattern during 6MWT most probably is different between patients with COPD and healthy elderly subjects [8].

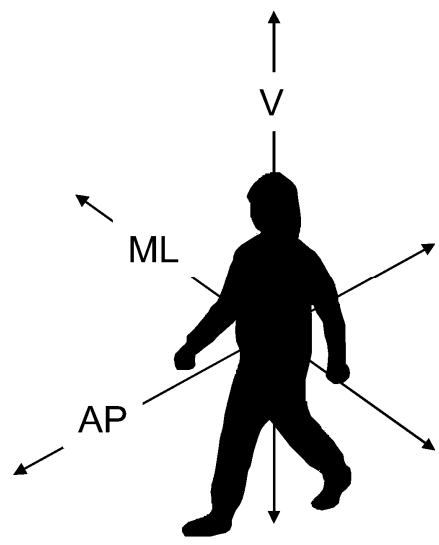

Figure 4.1. Directions of displacement. AP: Anterior-posterior direction (forward-backward displacement), V: Vertical direction (up-down displacement), ML: medio-lateral direction (left-right displacement) 
Detailed analyses of walking patterns during the 6MWT have not yet been performed in patients with COPD. Yentes et al. recently reported gross walking abnormalities in patients with COPD, such as the presence of a limp or shuffle [9]. These authors used qualitative assessment of gait abnormalities and did not asses the spatiotemporal aspects of walking abnormalities. The latter would enable a direct comparison of walking pattern between patients with COPD and healthy elderly subjects; and an examination of the association between walking pattern, 6MWD and clinical characteristics, like weight, height, the degree of airflow limitation and exercise-induced symptoms of dyspnea and fatigue.

Features derived from tri-axial accelerometers attached to the lower back can be used to measure walking variability. Accelerometers may also allow monitoring walking abnormalities in patients with COPD, as was done before in patients with chronic heart failure [10]. Moreover, routine assessment of exercise performance in a home-based setting in the context of telemedicine seems possible when close associations between accelerometer features and walking distance are found. Therefore, the aim of this study was to determine walking patterns during the 6MWT of COPD patients and healthy elderly subjects. A priori, the authors hypothesized that patients with COPD have a different walking pattern during 6MWT compared to healthy elderly subjects, which is related to the reduced 6MWD in COPD independent of the degree of airflow limitation.

\section{Methods}

\section{Participants}

Patients were recruited prospectively during a three-day pre-rehabilitation assessment period at $\mathrm{CIRO}+$, a center of expertise for chronic organ failure in Horn, the Netherlands [11]. Exclusion criteria were exacerbation-related hospitalization within 4 weeks prior to assessment and the use of a rollator, which is expected to affect the walking pattern [12]. Moreover, patients were excluded from analyses if they were not able to walk at least one 6MWT continuously for six minutes. In all cases non-continuous walks resulted in a worse 6MWD. This is necessary to obtain reliable measures of walking variability [13]. Moreover walking variability cannot be measured over non-walking time.

In total, 93 patients enrolled, of which 14 (15\%) stopped during both 6MWT. Therefore, 79 COPD patients were included in the analyses ( $n=8$ GOLD 1, $n=36$ GOLD 2, $n=28$ GOLD 3, n=7 GOLD 4). None of the remaining patients received long-term oxygen therapy (LTOT). All measurements were part of routine baseline assessment for pulmonary rehabilitation [11]. Furthermore, 24 healthy elderly subjects were 
recruited. Healthy volunteers were recruited amongst healthy subjects who participated in previous trials [14]. None of the healthy subjects used physician-prescribed drugs. The study complied with the Declaration of Helsinki and was approved by the local university's ethics committee (NL30763.068.09). Informed consent was provided by all participants.

\section{Study protocol}

Participants performed two 6MWTs on consecutive days [15]. During both tests an accelerometer (Minimod, McRoberts, The Hague, The Netherlands; size: $8.5 \times 5.0 \mathrm{x}$ $1.0 \mathrm{~cm}$, weight: $70 \mathrm{~g},+/-2 \mathrm{G}, 100 \mathrm{~Hz}$ sampling frequency) was attached to the trunk at the level of the sacrum using an elastic belt to collect raw signalling data. Data obtained during the 6MWT resulting in the highest distance were used for further analyses. Prior to and immediately after each 6MWT participants were asked to report dyspnea and fatigue on a ten-point Borg scale. The best 6-minute walk distance was expressed as a percentage of the predicted values [16].

Post-bronchodilator forced expiratory volume in the first second (FEV1) and forced vital capacity (FVC) were determined using spirometry and reference values were from Quanjer et al. [17]. Moreover, in the COPD patients residual volume (RV) and total lung capacity (TLC) were determined using a whole-body plethysmography to calculate the RV/TLC ratio as a measure of air-trapping. Height and weight were assessed to obtain body mass index (BMI, body weight in kilograms divided by squared height in meters, $\mathrm{kg} / \mathrm{m}^{2}$ ). Patients also underwent physical examination and medical history [18], Bio-electrical impedance analysis was used (Bodystat 1500 ) to determine fat-free mass (FFM) and disease specific equations were used to calculate fat-free mass index (FFMI) [19].

\section{Data Analysis}

For data analyses of the accelerometer signals 5 seconds at the beginning and end of the test were excluded to be sure that possible group differences in walking pattern were not due to start and/or stop of the 6MWT. Dedicated software written in $M A T L A B(c)$ was used to analyse the remaining 350 seconds of raw acceleration data. The software included algorithms to calculate the walking intensity, spatiotemporal aspects of gait and medio-lateral stability. Walking intensity was calculated from the integral of the modulus accelerometer output [20]. For this purpose, accelerometer output was low-pass filtered with a fourth-order Butterworth filter $(20 \mathrm{~Hz})$. The absolute value of the residual signal was taken to rectify the signal. After this process, the area under the curve over the complete measurement was calculated by integrating the signal over a period of 350 seconds. This integration was done separately for all three measurement directions (i.e. frontal, horizontal and sagittal plane). The integral of the modulus accelerometer output was then 
obtained by summation of these values [20]. Onsets of support phases were determined from forward accelerations as described by Zijlstra et al. [21]. During the transition from single to double support (i.e. after contra-lateral foot contact), the forward acceleration of the lower trunk changes sign from positive to negative. The peak forward acceleration preceding the change of sign coincides with the instant of foot contact. The acceleration peak preceding a change of sign (from positive to negative) was taken as the instant of a left or right foot contact. Consequently the strides (=2 steps) were identified. The cadence (strides/min) was calculated from the mean stride times. The inter-stride trunk acceleration variability was calculated using an unbiased autocorrelation coefficient procedure [22], which previously has been used to identify walking patterns of frail and fatigued elderly $[5,22]$. The autocorrelation function estimates how a time series is correlated with itself over different time lags. For a time series of trunk accelerations during walking, autocorrelation coefficients can thus be produced to quantify the peak values at the first and second dominant period, representing phase shifts equal to one step and one stride, respectively. Variability, as measured by the autocorrelation coefficients were calculated for the anterior-posterior, vertical and medio-lateral direction [23]. A higher autocorrelation coefficient indicates lower between-stride time variability (range: 0 to $100 \%)$.

\section{Sample Size and Power}

Sample size and power sample size calculations are based on outcomes of MoeNilsson et al. using the interstride trunk acceleration variability of fit and frail older adults [22]. Twenty-two participants in each group would provide $80 \%$ power at alpha 0.05 (two-tailed) to detect differences between COPD patients and healthy subject of $8 \%$ with a standard deviation of $10 \%$. To cover a larger spectrum of COPD severity by having enough patients in disease stages 1/2 and 3/4, 93 COPD patients enrolled the study.

\section{Statistical Analysis}

Statistical analysis was done using SPSS software (version 15.0, SPSS Inc.). Data are reported as mean \pm standard deviation (SD) or percentages, as appropriate. GOLD stages 1 and 2, and GOLD stages 3 and 4 were combined for further analyses due to the small number of GOLD stage $1(n=8)$ and GOLD stage 4 patients $(n=7)$. The comparisons were conducted with 1-way analysis of variance or chi-square tests as appropriate. Accelerometer features (walking intensity, cadence, variability in anterior-posterior, vertical and medio-lateral direction), subject characteristics (gender, age, height, weight and FEV1) and perceived dyspnea and fatigue (before and after the best 6MWT) were tested in their association with the 6MWD via multiple ordinary least squares (OLS) regression models per group. Previously, different patterns were found for the variability in medio-lateral direction versus anterior-posterior 
and vertical directions between frail or fatigued persons and fit persons [5,22]. Therefore the interactions between variability for the different directions were also tested. Multicollinearity tests were carried out variables were retained in the model if the variance inflation factor was smaller than 5.0. A top-down procedure was handled for the selection of the final model variables. Accelerometer features probably rely, at least in part, on the walking speed. Therefore, a posteriori walking variability measures were compared in a subset of healthy subjects and COPD patients who had on average a comparable walking distance (range 6MWD: 560m-640m). A priori, results were considered statistically significant when $p$-value was $\leq 0.05$.

\section{Results}

\section{Characteristics}

Healthy subjects and COPD patients had a similar gender distribution, age and BMI (table 4.1). As expected, GOLD stage 3/4 patients had the worst 6MWD, also after correction for confounding variables, like height, weight, age and gender [16]. COPD patients experienced more fatigue and dyspnea during the 6MWT compared to healthy elderly subjects. 
Table 4.1: Patients' characteristics

\begin{tabular}{|c|c|c|c|c|}
\hline & $\begin{array}{l}\text { Healthy } \\
(n=24)\end{array}$ & $\begin{array}{l}\text { All COPD } \\
(n=79)\end{array}$ & $\begin{array}{l}\text { GOLD 1/2 } \\
\qquad(n=44)\end{array}$ & $\begin{array}{c}\text { GOLD 3/4 } \\
(n=35)\end{array}$ \\
\hline Men (\%) & 62.5 & 59.5 & 59.1 & 60 \\
\hline Age (yrs) & $63.7 \pm 5.9$ & $64.3 \pm 8.9$ & $64.9 \pm 8.5$ & $63.5 \pm 9.5$ \\
\hline Height (m) & $1.73 \pm 0.07$ & $1.67 \pm 0.09^{*}$ & $1.67 \pm 0.10^{*}$ & $1.66 \pm 0.06^{*}$ \\
\hline Weight (kg) & $79.5 \pm 13.2$ & $69.0 \pm 15.0^{*}$ & $70.1 \pm 16.3^{*}$ & $67.5 \pm 13.2^{*}$ \\
\hline BMI $\left(\mathrm{kg} / \mathrm{m}^{2}\right)$ & $25.8 \pm 3.8$ & $24.7 \pm 4.5$ & $25.0 \pm 4.6$ & $24.3 \pm 4.3$ \\
\hline Tiffeneau index (\%) & $77.0 \pm 4.1$ & $40.7 \pm 11.9^{*}$ & $47.6 \pm 9.3^{*}$ & $31.9 \pm 8.4^{*} \#$ \\
\hline FEV1 (\%pred) & $124.9 \pm 21.0$ & $53.5 \pm 18.7^{*}$ & $66.6 \pm 14.0^{*}$ & $37.0 \pm 7.2 * \#$ \\
\hline RV/TLC ratio (\%) & - & $50.5 \pm 10.0$ & $45.6 \pm 8.9$ & $56.8 \pm 7.5 \#$ \\
\hline 6MWD (m) & $672 \pm 85$ & $494 \pm 96 *$ & $528 \pm 95^{*}$ & $451 \pm 79 * \#$ \\
\hline 6MWD (\% pred) & $102.3 \pm 11.6$ & $77.6 \pm 13.5^{*}$ & $83.4 \pm 12.0^{*}$ & $70.3 \pm 11.7^{*} \#$ \\
\hline Baseline dyspnea (points) & $0.31 \pm 0.51$ & $1.64 \pm 1.22^{*}$ & $1.34 \pm 1.19 *$ & $2.01 \pm 1.18^{*} \#$ \\
\hline$\Delta$ Dyspnea (points) & $1.33 \pm 1.33$ & $2.74 \pm 1.97^{*}$ & $2.77 \pm 2.04^{*}$ & $2.70 \pm 1.91^{*}$ \\
\hline Baseline fatigue (points) & $0.50 \pm 0.81$ & 1. $52 \pm 1.48^{*}$ & $1.41 \pm 1.53^{*}$ & $1.66 \pm 1.42^{*}$ \\
\hline$\Delta$ Fatigue (points) & $1.13 \pm 1.27$ & $2.25 \pm 1.76^{*}$ & $2.22 \pm 1.52^{*}$ & $2.30 \pm 2.04^{*}$ \\
\hline FFM (kg) & - & $46.5 \pm 8.8$ & $47.3 \pm 9.1$ & $45.6 \pm 8.5$ \\
\hline FFMi $\left(\mathrm{kg} / \mathrm{m}^{2}\right)$ & - & $16.6 \pm 2.2$ & $16.8 \pm 2.1$ & $16.3 \pm 2.3$ \\
\hline
\end{tabular}

Abbreviations: BMI: body mass index, FEV1: forced expiratory volume in the first second, RV: residual volume,TLC: total lung capacity, 6MWD: 6-min walk distance, FFM: fat-free mass, FFMi: fat-free mass index.

*: significantly different from healthy subjects. $(p<0.05)$

\#: significantly different from GOLD stage $1 / 2$. ( $p<0.05)$ 


\section{Accelerometer features}

Accelerometer features showed that COPD patients walked at a significantly lower intensity and a lower cadence (table 4.2). Differences in intensity and cadence were also found between patients in GOLD stages $3 / 4$ and GOLD stages $1 / 2$. Moreover significantly increased variability (as measured by the lower autocorrelation coefficients) was found for the medio-lateral acceleration in the COPD group compared to healthy controls.

Table 4.2: Accelerometer features

\begin{tabular}{lcccc}
\hline & $\begin{array}{c}\text { Healthy } \\
(\mathrm{n}=24)\end{array}$ & $\begin{array}{c}\text { COPD } \\
(\mathrm{n}=79)\end{array}$ & $\begin{array}{c}\text { GOLD 1/2 } \\
(\mathrm{n}=44)\end{array}$ & $\begin{array}{c}\text { GOLD 3/4 } \\
(\mathrm{n}=35)\end{array}$ \\
\hline $\begin{array}{l}\text { Walking intensity } \\
\text { (counts/min) }\end{array}$ & $14054 \pm 3198$ & $8658 \pm 2971^{*}$ & $9892 \pm 3214^{*}$ & $7106 \pm 1654^{*} \#$ \\
Cadence (strides/min) & $66 \pm 4$ & $57 \pm 6^{*}$ & $59 \pm 5^{*}$ & $55 \pm 5^{*} \#$ \\
AC-AP (\%) & $81.9 \pm 10.4$ & $79.0 \pm 10.7$ & $79.4 \pm 9.7$ & $78.5 \pm 12.0$ \\
AC-V (\%) & $87.3 \pm 6.9$ & $84.2 \pm 10.2$ & $85.6 \pm 6.8$ & $82.5 \pm 13.3$ \\
AC-ML (\%) & $73.7 \pm 12.5$ & $63.2 \pm 14.0^{*}$ & $64.4 \pm 12.4^{*}$ & $61.6 \pm 15.9^{*}$ \\
\hline
\end{tabular}

Abbreviations: AC-AP: autocorrelation coefficient in anterior-posterior direction, $\mathrm{AC}-\mathrm{V}$ : autocorrelation coefficient in vertical direction, AC-ML: autocorrelation coefficient in medio-lateral direction

*: significantly different from healthy subjects. $(p<0.05)$

\#: significantly different compared to GOLD 1/2. $(p<0.05)$

\section{A posteriori analysis}

Healthy subjects $(n=8)$ and COPD patients $(n=14)$ with a 6MWD range between $560 \mathrm{~m}$ and $640 \mathrm{~m}$ did not differ significantly in 6MWD (healthy: $595 \pm 13 \mathrm{~m}$, COPD: $596 \pm 20 \mathrm{~m}, \mathrm{p}=0.869$ ), walking intensity (healthy: $11782 \pm 1083$ a.u., COPD: $11501 \pm 2013$ a.u., $p=0.720$ ) and cadence (healthy: $64 \pm 3$ strides/min, COPD: $61 \pm 4$ strides/min, $p=0.107)$. Nevertheless, the COPD patients maintained to have a significantly lower autocorrelation coefficient in the medio-lateral direction compared to the healthy control subjects (healthy: $81 \pm 7 \%$, COPD: $67 \pm 12 \%, p=0.003$ ).

\section{Determinants of $6 M W D$}

The intensity parameter walking intensity correlated most strongly with the 6MWD in healthy subjects $(r=0.902, p<0.001)$ and COPD patients $(r=0.872, p<0.001)$ (figure 4.2). The correlation between 6MWD and FEV1 in COPD $(r=0.452, p<0.001)$ and between walking intensity and FEV1 in COPD ( $r=0.495, p<0.001)$ were both significant. No significant correlations between these variables were found in healthy subjects. 

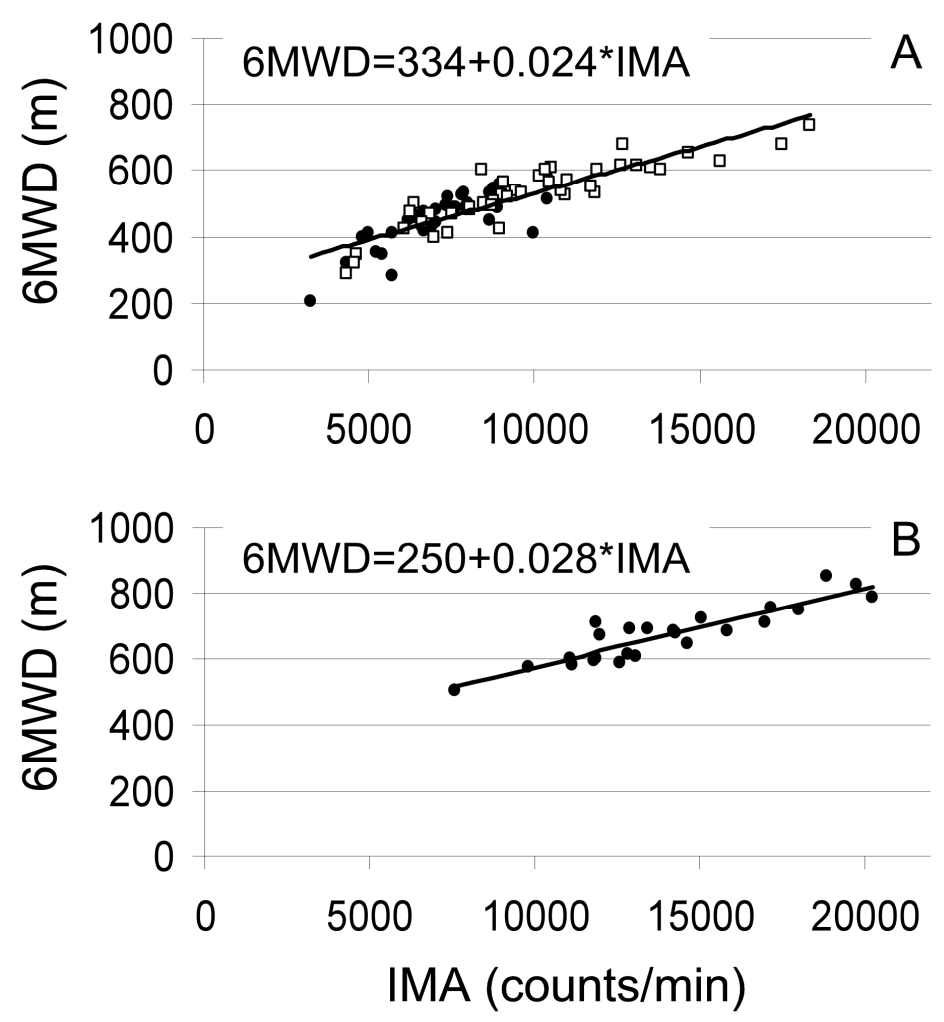

Figure 4.2. Relation between the accelerometer output walking intensity per min and the 6MWD. A: GOLD stages 1 and 2 with open squares and GOLD stages 3 and 4 with closed circles and B: Healthy subjects

The results of the OLS regression models used to test associations between subject characteristics, accelerometer features and 6MWD for healthy subjects and COPD patients (all GOLD stages) are summarised in table 4.3. The model variables explained $85 \%$ and $88 \%$ of the variability in 6MWD in healthy subjects and COPD patients, respectively. Walking intensity and height were the only two significant determinants of 6MWD in healthy elderly subjects. In patients with COPD also age, cadence, walking variability measures and their interactions were included. 
Table 4.3: Model variables influencing the 6MWD - Linear Regression

\begin{tabular}{lll}
\hline & \multicolumn{1}{c}{$\begin{array}{c}\text { Healthy } \\
(\mathrm{n}=24)\end{array}$} & \multicolumn{1}{c}{$\begin{array}{c}\text { COPD } \\
(\mathrm{n}=79)\end{array}$} \\
\hline Height (m) & $242.6[54.4-430.3]$ & $244.8[132.4-357.2]$ \\
Age (y) & $\mathrm{NS}$ & $-1.03[-2.02--0.04]$ \\
Walking intensity & $0.023[0.018-0.028]$ & $0.019[0.016-0.023]$ \\
(counts/min) & $\mathrm{NS}$ & $3.01[0.81-5.20]$ \\
Cadence (strides/min) & $\mathrm{NS}$ & $15.15[5.30-24.99]$ \\
AC-AP (\%) & $\mathrm{NS}$ & $10.19[4.28-16.11]$ \\
AC-V (\%) & $\mathrm{NS}$ & $-10.84[-17.09--4.60]$ \\
AC-ML (\%) & $\mathrm{NS}$ & $-0.18[-0.30--0.06]$ \\
AC-AP*AC-V (interaction) & $\mathrm{NS}$ & $0.12[0.05-0.19]$ \\
AC-V*AC-ML (interaction) & $-70.82[-391.68-250.04]$ & $-991.1[-1491.9--490.26]$ \\
Model Constant & 0.85 & 0.88 \\
Adjusted Model R & & \\
\hline
\end{tabular}

Value expressed as Beta's $(B) \pm 95 \%$ confidence interval $[\mathrm{Cl}]$

Abbreviations: AC-AP: autocorrelation coefficient in anterior-posterior direction, AC-V: autocorrelation coefficient in vertical direction, AC-ML: autocorrelation coefficient in medio-lateral direction. NS: not significant

Note: effects of the following variables were also tested, but no statistical significance was detected: Gender, weight, FEV1, perceived dyspnea and fatigue (before - after the 6MWT), interaction between the autocorrelation coefficient in anterior-posterior and medio-lateral direction.

\section{Discussion}

The present study provides the first comprehensive evaluation of qualitative and quantitative measures of the walking pattern during 6MWT in patients with moderate to very severe COPD. It extends previous work on the 6MWT by providing detailed information on walking variability. On average, COPD patients walk with a lower intensity, a lower cadence and show a higher medio-lateral variability during 6MWT in comparison with healthy elderly subjects. The difference in medio-lateral variability remained even if the walking speed was similar. Moreover, walking variability was associated with functional exercise capacity in COPD patients, but not in healthy controls. These results indicate an altered walking pattern in COPD patients.

Increased variability in the medio-lateral direction (largely due to through lateral foot placement) is an active control strategy to compensate for balance disturbances in order to maintain stability in the anterior-posterior direction (the direction of propulsion). In the present study, walking variability was higher in the me- 
dio-lateral direction in COPD patients compared to the control group (table 4.2), suggesting larger balance disturbances during the 6MWT in the patients. This may at least in part contribute to the relatively high energetic costs of a 6MWT in patients with COPD [24]. Moreover, this may also explain partially why patients with COPD experience abnormalities with day-to-day walking [25], including falls [26]. Indeed similar deviations in walking patterns were previous observed in frail elderly who fell at least once during the last year or who used walking aid [22].

A high positive association was found between the intensity parameter walking intensity and 6MWD in both COPD patients and healthy controls (figure 4.2). Previously, similar findings were reported in patients with chronic heart failure $[10,27]$. These high associations create future possibilities for routine assessment of exercise performance of patients in a home-based setting in the context of telemedicine. Moreover, multiple accelerometer features and subject characteristics explained $88 \%$ of the variability in 6MWD in the patients with COPD. Next to familiar determinants of the 6MWD in COPD (i.e., height and age), also a higher walking intensity, a higher cadence, lower variability in anterior-posterior and vertical directions, and a higher variability in medio-lateral direction were significantly associated with a higher 6MWD (table 4.3). Moreover, interactions between variability in anteriorposterior and vertical direction and between vertical en medio-lateral direction were found. The degree of airflow limitation (e.g., FEV1) did not significantly explain the variance in 6MWD in a multiple model.

Previously, the cadence and the walking intensity have been studied in patients' home-environments to evaluate daily performance in COPD patients or to study the effects of pulmonary rehabilitation [28]. The present study shows that walking variability is also a clinically relevant variable in COPD patients as it significantly contributes to the prediction of 6MWD. This is an important finding as lower 6MWD has been related to more exacerbation-related hospitalizations and higher mortality rates in patients with COPD [2].

The design of this study was cross-sectional. Future investigations using the current methodology should include repeated measures to investigate the effect of a comprehensive pulmonary rehabilitation program on walking pattern in patients with COPD. Indeed, faster walking may be more stable than slow walking [29]. On the other hand, this study showed that also the COPD patients with the same walking speed as healthy subjects have an increased variability in the medio-lateral direction. This strongly suggests that the differences found in walking variability between COPD and healthy cannot be attributed only to differences in walking speed. It is therefore more likely that in COPD patients walking stability is influenced by dyspnea [30], altered breathing dynamics [31], reduced arm swing [32], lower muscle 
strength and/or coordination [5,6], disturbed balance [7,33], or a combination thereof. Accelerometers may be helpful to evaluate 6-min walking patterns as an index of treatment outcome. Moreover current findings should be reproduced in the patients own environment.

Participants who stopped during both 6MWT were excluded from this study, which was necessary to obtain reliable measures of walking variability [13]. As a result, the 6MWD of the COPD patients was higher compared to previous studies [15,34]. Then again, the current mean 6MWD of $494 \mathrm{~m}$ is well within the range in 6WMD as observed in the ECLIPSE study [3]. The Modified Medical Research Council (MMRC) Dyspnea Scale was not assessed, therefore it cannot be excluded that dyspnea may have contributed to the 6MWD in patients with COPD [3]. The present results are hypothesis-generating rather than definitive. Future studies are warranted to corroborate the present findings and to explain why patients with COPD have a different walking pattern compared to healthy elderly subjects. This may be due to a variety of factors $[5,6,7]$. Moreover, the current findings generate a clear rationale to study in detail walking patterns using tri-dimensional analyses, including electromyographic activity of lower-limb muscles [35].

In conclusion, patients with COPD have a different walking pattern during 6MWT compared to healthy elderly subjects, as objectified by using accelerometer signals. In addition to walking intensity, cadence and walking variability are important variables associated with 6MWD in patients with COPD. These differences in walking pattern partially explain the reduction in 6MWD in patients with COPD.

\section{Acknowledgements}

The authors are grateful to the volunteers who participated in the present study. 


\section{References}

1. (2002) ATS statement: guidelines for the six-minute walk test. Am J Respir Crit Care Med 166: 111117.

2. Spruit MA, Polkey MI, Celli B, Edwards LD, Watkins ML, et al. (2011) Predicting Outcomes from 6Minute Walk Distance in Chronic Obstructive Pulmonary Disease. J Am Med Dir Assoc 13: 291-297.

3. Spruit MA, Watkins ML, Edwards LD, Vestbo J, Calverley PM, et al. (2010) Determinants of poor 6min walking distance in patients with COPD: the ECLIPSE cohort. Respir Med 104: 849-857.

4. Donelan JM, Kram R, Kuo AD (2001) Mechanical and metabolic determinants of the preferred step width in human walking. Proc Biol Sci 268: 1985-1992.

5. Helbostad JL, Leirfall S, Moe-Nilssen R, Sletvold O (2007) Physical fatigue affects gait characteristics in older persons. J Gerontol A Biol Sci Med Sci 62: 1010-1015.

6. Seymour JM, Spruit MA, Hopkinson NS, Natanek SA, Man WD, et al. (2010) The prevalence of quadriceps weakness in COPD and the relationship with disease severity. Eur Respir J 36: 81-88.

7. Smith MD, Chang AT, Seale HE, Walsh JR, Hodges PW (2010) Balance is impaired in people with chronic obstructive pulmonary disease. Gait Posture 31: 456-460.

8. Kuo AD, Donelan JM (2010) Dynamic principles of gait and their clinical implications. Phys Ther 90 : 157-174.

9. Yentes JM, Sayles H, Meza J, Mannino DM, Rennard SI, et al. (2011) Walking abnormalities are associated with COPD: An investigation of the NHANES III dataset. Respir Med 105: 80-87.

10. Jehn M, Schmidt-Trucksaess A, Schuster T, Hanssen H, Weis M, et al. (2009) Accelerometer-based quantification of 6-minute walk test performance in patients with chronic heart failure: applicability in telemedicine. J Card Fail 15: 334-340.

11. Spruit MA, Vanderhoven-Augustin I, Janssen PP, Wouters EF (2008) Integration of pulmonary rehabilitation in COPD. Lancet 371: 12-13.

12. Alkjaer T, Larsen PK, Pedersen G, Nielsen LH, Simonsen EB (2006) Biomechanical analysis of rollator walking. Biomed Eng Online 5: 2.

13. Kang HG, Dingwell JB (2006) Intra-session reliability of local dynamic stability of walking. Gait Posture 24: 386-390.

14. Vaes AW, Wouters EF, Franssen FM, Uszko-Lencer NH, Stakenborg KH, et al. (2011) Task-related oxygen uptake during domestic activities of daily life in patients with COPD and healthy elderly subjects. Chest 140: 970-979.

15. Hernandes NA, Wouters EF, Meijer K, Annegarn J, Pitta F, et al. (2011) Reproducibility of 6-minute walking test in patients with COPD. Eur Respir J 38: 261-267.

16. Troosters T, Gosselink R, Decramer M (1999) Six minute walking distance in healthy elderly subjects. Eur Respir J 14: 270-274.

17. Quanjer PH, Tammeling GJ, Cotes JE, Pedersen OF, Peslin R, et al. (1993) Lung volumes and forced ventilatory flows. Report Working Party Standardization of Lung Function Tests, European Community for Steel and Coal. Official Statement of the European Respiratory Society. Eur Respir J Suppl 16: $5-40$.

18. Spruit MA, Pennings HJ, Janssen PP, Does JD, Scroyen S, et al. (2007) Extra-pulmonary features in COPD patients entering rehabilitation after stratification for MRC dyspnea grade. Respir Med 101: 2454-2463.

19. Steiner MC, Barton RL, Singh SJ, Morgan MD (2002) Bedside methods versus dual energy X-ray absorptiometry for body composition measurement in COPD. Eur Respir J 19: 626-631.

20. Bouten CV, Sauren AA, Verduin M, Janssen JD (1997) Effects of placement and orientation of bodyfixed accelerometers on the assessment of energy expenditure during walking. Med Biol Eng Comput 35: 50-56. 
21. Zijlstra W (2004) Assessment of spatio-temporal parameters during unconstrained walking. Eur J Appl Physiol 92: 39-44.

22. Moe-Nilssen R, Helbostad JL (2005) Interstride trunk acceleration variability but not step width variability can differentiate between fit and frail older adults. Gait Posture 21: 164-170.

23. Moe-Nilssen R, Helbostad JL (2004) Estimation of gait cycle characteristics by trunk accelerometry. J Biomech 37: 121-126.

24. Troosters T, Vilaro J, Rabinovich R, Casas A, Barbera JA, et al. (2002) Physiological responses to the 6min walk test in patients with chronic obstructive pulmonary disease. Eur Respir J 20: 564-569.

25. Annegarn J, Meijer K, Passos VL, Stute K, Wiechert J, et al. (2011) Problematic Activities of Daily Life are Weakly Associated With Clinical Characteristics in COPD. J Am Med Dir Assoc 13: 284-290.

26. Roig M, Eng JJ, Maclntyre DL, Road JD, FitzGerald JM, et al. (2011) Falls in people with chronic obstructive pulmonary disease: an observational cohort study. Respir Med 105: 461-469.

27. Annegarn J, Spruit MA, Uszko-Lencer NH, Vanbelle S, Savelberg HH, et al. (2011) Objective physical activity assessment in patients with chronic organ failure: a validation study of a new single-unit activity monitor. Arch Phys Med Rehabil 92: 1852-1857 e1851.

28. Pitta F, Troosters T, Probst VS, Langer D, Decramer M, et al. (2008) Are patients with COPD more active after pulmonary rehabilitation? Chest 134: 273-280.

29. Bruijn SM, van Dieen JH, Meijer OG, Beek PJ (2009) Is slow walking more stable? J Biomech 42: 15061512.

30. Celli BR, Cote CG, Marin JM, Casanova C, Montes de Oca M, et al. (2004) The body-mass index, airflow obstruction, dyspnea, and exercise capacity index in chronic obstructive pulmonary disease. N Engl J Med 350: 1005-1012.

31. O'Donnell D E, Laveneziana P (2006) Physiology and consequences of lung hyperinflation in COPD. Eur Respir Rev 15: 61-67.

32. Ortega JD, Fehlman LA, Farley CT (2008) Effects of aging and arm swing on the metabolic cost of stability in human walking. J Biomech 41: 3303-3308.

33. Verghese J, Holtzer R, Lipton RB, Wang C (2009) Quantitative gait markers and incident fall risk in older adults. J Gerontol A Biol Sci Med Sci 64: 896-901.

34. Janssen DJ, Spruit MA, Leue C, Gijsen C, Hameleers H, et al. (2010) Symptoms of anxiety and depression in COPD patients entering pulmonary rehabilitation. Chron Respir Dis 7: 147-157.

35. Marquis N, Debigare R, Bouyer L, Saey D, Laviolette L, et al. (2009) Physiology of walking in patients with moderate to severe chronic obstructive pulmonary disease. Med Sci Sports Exerc 41: 15401548. 



\section{Chapter 5}

\section{Assessment of intensity and elevation of arm movements during activities of daily life}

Janneke Annegarn, Martijn A. Spruit, Annemie M.W.J. Schols, Hans H.C.M. Savelberg, Alessio Murgia, Emiel F.M. Wouters and Kenneth Meijer.

Submitted for publication 


\begin{abstract}
Arm activity monitors can provide an objective, real world index of upper-limb use for various populations and objectives. The aim of this study was to develop a classification scheme of intensity and elevation of arm movements. Secondary aims are to assess the optimal sensor placement, to investigate the validity of this placement and to evaluate arm activities of daily living ( $A D L)$. Ten participants wearing tri-axial accelerometers placed on the wrist, elbow and shoulder performed arm activities at three levels of arm elevation and intensity. The overall elevation and intensity classification accuracy was clearly highest for the elbow location (wrist sensor: $43 \pm 12 \%$; elbow sensor: $99 \pm 19 \%$; shoulder sensor: $44 \pm 12 \%$ ). A two-day arm ADL assessment in another ten participants revealed that most time is spent at a low arm intensity and low elevation (404 min/day) and only $5 \mathrm{~min} /$ day at a high intensity and high elevation. Arm elevation and intensity are important to assess as they combined provide both quantitative and qualitative information on functional use. Only the elbow sensor resulted in excellent classification. All categories are manifest during normal daily arm use. These results give confidence that single-unit accelerometers can provide an objective, real world index of upper-limb use.
\end{abstract}




\section{Introduction}

Whole-body physical function is an important determinant of health and a primary focus of many studies [1]. More specifically, good arm function is extremely important for quality of life and independence during day-to-day life [2]. Due to the variety and complexity of arm movements, assessment of arm function has been a major challenge. With the vast collection of outcome measures available, choosing an appropriate instrument can be difficult. Researchers have proposed complex frameworks for detailed marker-based motion analysis of the upper body [3]. On the other hand, multiple less-accurate scales and checklist are available to quantify functional outcomes [4]. Monitoring arm activity profiles in the persons own environment better reflects reality and can be used to continuously evaluate progression and decline of functional arm use over time.

Wearable sensors can provide an continuous objective, real world index of arm use in patients $[5,6,7,8]$. The combination of multiple sensors (e.g. accelerometers, gyroscopes magnetometers) allows assessment of three-dimensional kinematics in the subjects' living environment. Detailed kinematics of the scapulothoracic [9], the humerothoracic $[10]$, the shoulder $[6,11]$ the elbow $[10,12,13,14]$ and the wrist $[15]$ has been described. To estimate the joint position or movement of interest, data supplied by the different sensor systems are usually combined through sensorfusion algorithms and subsequently used as input for biomechanical models describing the arm movements. While these sophisticated methods are highly accurate, they also have some important limitations. They require a large power supply, which makes them bulky, heavy and shortens their battery life, thus limiting their use to long-term monitoring activities of daily living (ADLs). Furthermore, some systems are too cumbersome for ADL monitoring due to the wire connections between sensors and they may be susceptible to magnetic distortion [16].

For many clinical purposes, including long term evaluation of daily arm use, the necessity of multiple sensors is questionable as less detailed information on arm use could be sufficient to monitor arm movement intensity and elevation. Indeed, arm movement intensity and elevation are important sensor outputs as they provide both quantitative and qualitative information of functional arm use $[15,17,18,19]$. To date, however, no method is available that classifies the combination of intensity and elevation of arm movements for longer periods of time using a single-unit accelerometer. Accelerometers show good validity for detecting arm movement intensity $[5,20]$. However, during the assessment of arm elevation small errors are introduced by any linear acceleration besides gravity [21,22]. The magni- 
tude of these errors is influenced by sensor position. It is currently unclear whether these errors impede monitoring of arm movement intensity and elevation during ADLs. Therefore the primary aim of this study was to develop a classification scheme of intensity and elevation of arm movements. In addition, we aimed to assess the optimal placement of a tri-axial accelerometer on the arm in terms of classification accuracy, and to investigate the associated validity of this placement to evaluate arm ADLs.

\section{Methods}

Two studies were designed. Study A to identify the optimal sensor position and develop and validate a classification scheme for arm use; and Study B to test whether the classification scheme was representative for arm use in daily life.

Study A: 10 healthy participants (5 men, age: $27 \pm 5$ yrs, height: $177 \pm 9 \mathrm{~cm}$, weight: $68 \pm 11 \mathrm{~kg}$ ) wore three sensors (tri-axial accelerometers) on the right arm: one sensor at the wrist, one at the elbow and one at the shoulder (SensorSleeve, Maastricht Instruments, figure 5.1). Each sensor contains a tri-axial piezoresistive accelerometer, weighs 15 gram $\left(1 * w * h: 15^{*} 15^{*} 5 \mathrm{~mm}\right)$, samples at $100 \mathrm{~Hz}$ and has a range of \pm 5 times gravity $(\mathrm{G})$ with a resolution of $0.02 \mathrm{G}$.

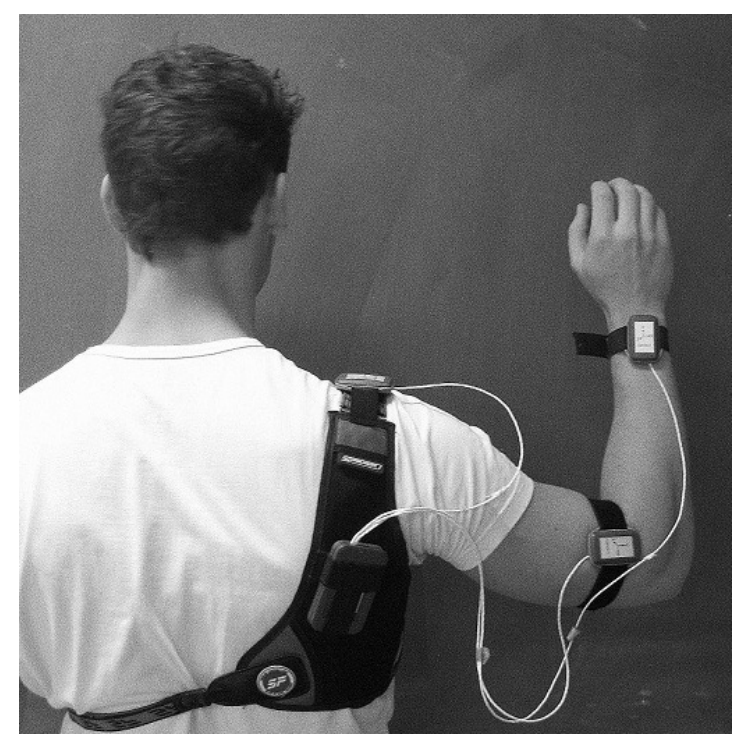

Figure 5.1: Subject wearing the sensor sleeve.

Participants were asked to stand in front of a marked wall while performing a standardized protocol on two separate days (figure 5.2). The wall was marked with three horizontal lines $30 \mathrm{~cm}$ apart. The height of the lines varied per subject in such way that when the subject placed his/her hand on the lines, the upper arm would 
have an elevation of $22.5^{\circ}$ (position 1 ), $67.5^{\circ}$ (position 2) and $112.5^{\circ}$ (position 3) relative to the longitudinal axes (figure 5.2). A goniometer was used to define the correct heights of the marks on the wall. After holding the arm still at the three different postures for 15 seconds the subjects were instructed to wave for $30 \mathrm{sec}$ onds at a metronome-paced speed of 84 beats per minute (bpm) with a range of 30 $\mathrm{cm}$ at the three different positions. This was repeated at $164 \mathrm{bpm}$. Thus in total participants performed on two separate days nine activities for 30 seconds at three levels of arm elevation (using marked wall) and at three levels of movement intensity (holding the arm still and metronome-paced).

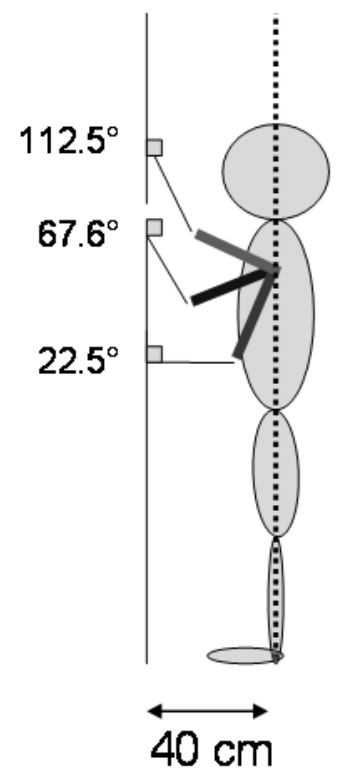

Figure 5.2: Schematic representation of a subject standing in front of the marked wall. Arm elevation was controlled by three marks on the wall, drawn for each subject in such way that the shoulder had an elevation of 1$\left.) 22.5^{\circ}, 2\right) 67.5^{\circ}$, 3) $112.5^{\circ}$ relative to the longitudinal axes (dotted line).

The first and last 5 seconds of accelerometer data of each activity were removed from further analyses to exclude transition time between activities. Dedicated MATLABC software and algorithms were used to calculate the signal magnitude area (SMA) as a measure of arm intensity and the mean low pass signal in the longitudinal direction (MI) as a measure of arm elevation [23]. Arm elevation and intensity were calculated over each second, to ensure good classification accuracy [6]. A hierarchical classification scheme was used to differentiate between three levels of arm elevation and arm intensity (figure 5.3). For this purpose four thresholds (2 for SMA and 2 for $\mathrm{MI}$ ) were determined to separate three levels of arm elevation and intensity for each accelerometer placement based on the data obtained from the protocol performed at day one only (figure 5.4 ). This was done by systematically 
shifting all thresholds by $1 \%$ steps. At each step the classification accuracy was defined as the average percentage of correctly classified seconds over all categories. Thresholds associated with the highest classification accuracy were tested using data collected at day two. Also for this purpose the classification accuracy were determined for each sensor placement. 95\% accuracy was defined as acceptable on forehand.

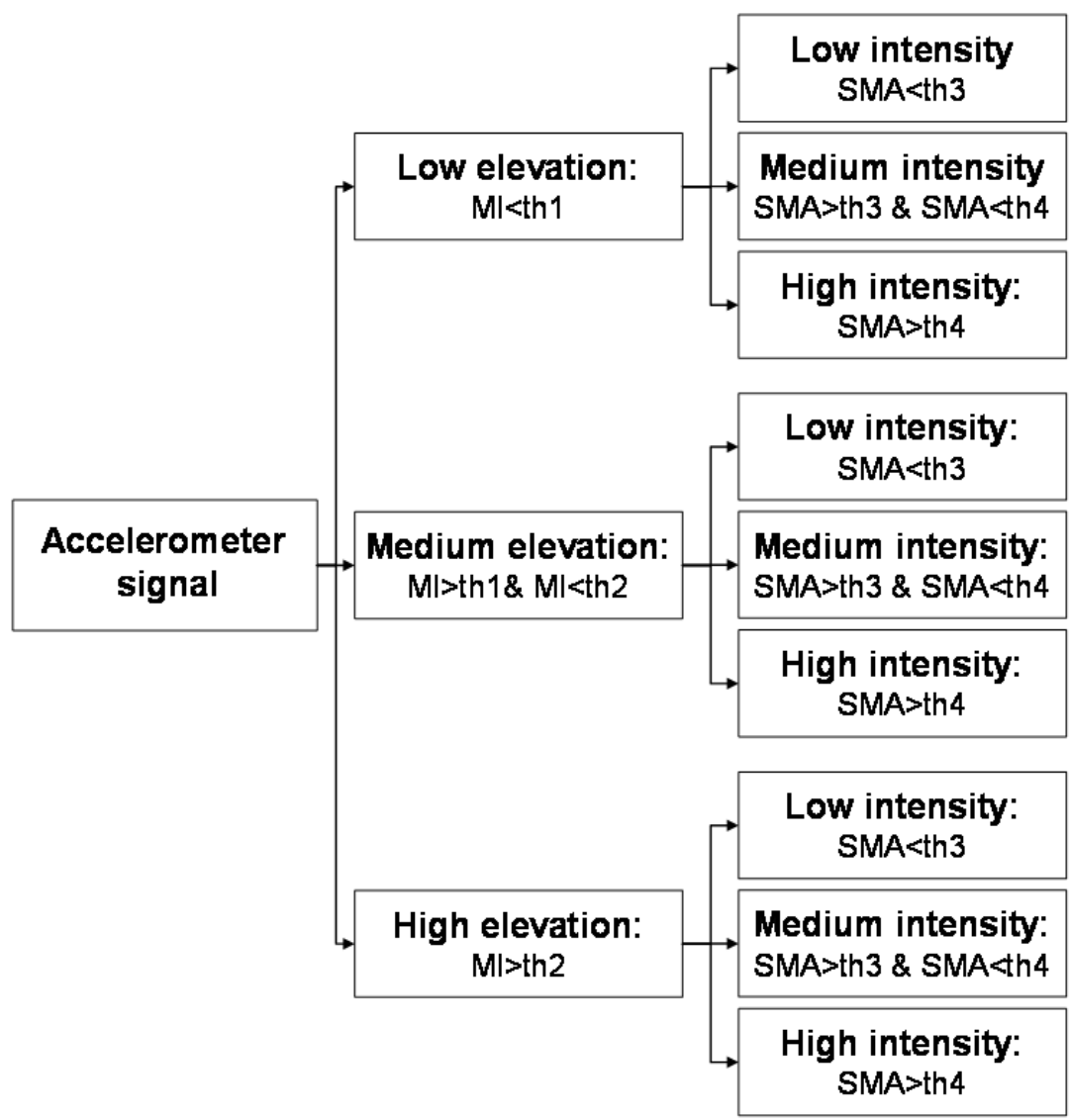

Figure 5.3: Arm activity classification scheme based on two thresholds (th) for arm elevation (MI) and two thresholds for arm intensity (SMA).

Study B: To assess the presence of arm categories defined in study A during ADL, 10 young healthy participants ( 5 men, age: $25 \pm 7$ yrs, height: $172 \pm 9 \mathrm{~cm}$, weight: $69 \pm 11$ $\mathrm{kg}$ ) were asked to wear a tri-axial piezoresistive accelerometer (CAM, Maastricht 
Instruments B.V., the Netherlands; samples: $25 \mathrm{~Hz}$, range: $\pm 4 \mathrm{G}$, resolution: $0.02 \mathrm{G}$ ). Participants wore the CAM on the optimal placement on the arm (as defined in study A), and on the leg simultaneously. Participants were measured on two consecutive days during waking hours. Participants were allowed to remove the CAMs while washing, swimming and sleeping.
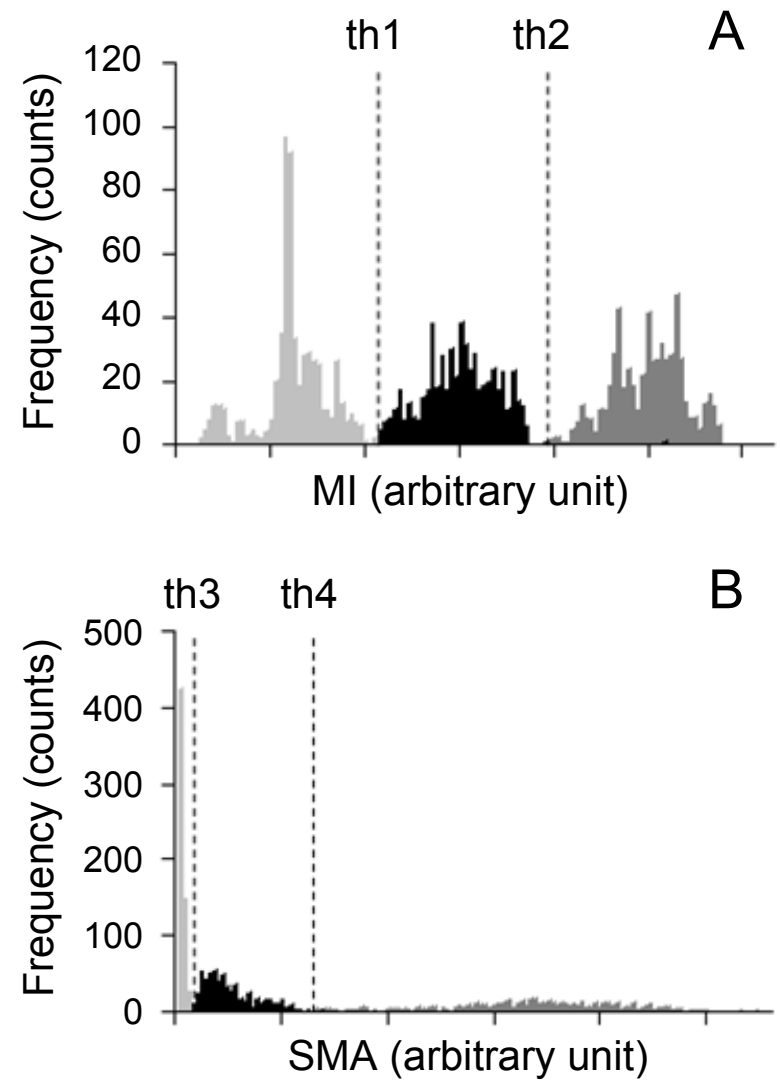

Figure 5.4: Frequency distribution of arm categories and thresholds associated with the highest classification accuracy. A: Frequency distribution of low elevation (light grey), medium elevation (black) and high elevation (dark grey) arm activities. Two thresholds were determined to classify three levels of arm elevation. B: Frequency distribution of low intensity (light grey), medium intensity (black) and high intensity (dark grey) arm activities. Two thresholds were determined to classify three levels of arm intensity.

The thresholds from study A were used to categorize three levels of arm elevation and three levels of arm intensity. An additional activity monitor fixed on the leg was used to differentiate between arm movement during ambulation or arm movements for object manipulation and gesturing during standing and sitting (Chapter 3). Daily arm activity was calculated for each period corresponding to standing, sitting and ambulation. Moreover the mean time participants spent per day within a certain arm category for the duration range 1-20 seconds was investigated. 


\section{Results}

Study A: The combined arm elevation and intensity classification accuracy (mean $\pm S D$ ) was $43.2 \pm 11.6 \%$ for the wrist sensor, $99.4 \pm 0.9 \%$ for the elbow sensor and $43.8 \pm 11.9 \%$ for the shoulder sensor. Arm movement intensity was classified correctly by all sensors (wrist sensor: $98.3 \pm 1.1 \%$, elbow sensor: $99.5 \pm 0.9 \%$, shoulder sensor: $95.6 \pm 3.7 \%)$. In contrast, arm elevation was only classified correctly by the elbow sensor (wrist sensor: $43.8 \pm 11.9 \%$, elbow sensor: $99.9 \pm 0.2 \%$, shoulder sensor: $41.4 \pm 10.1 \%)$.

Study B: Subjects wore the activity monitors on average $15.58 \pm 1.28$ hours/day. All arm categories were present in daily life (figure 5.5). Participants spent most time at a low arm elevation and low arm intensity (404 $\pm 12 \mathrm{~min} /$ day) and least time at a high arm elevation and high arm intensity ( $5 \pm 1 \mathrm{~min} /$ day). High intensity arm ADLs were most present during ambulation while low intensity arm ADLs were most present during sitting (figure 5.5).

\section{Shoulder elevation}

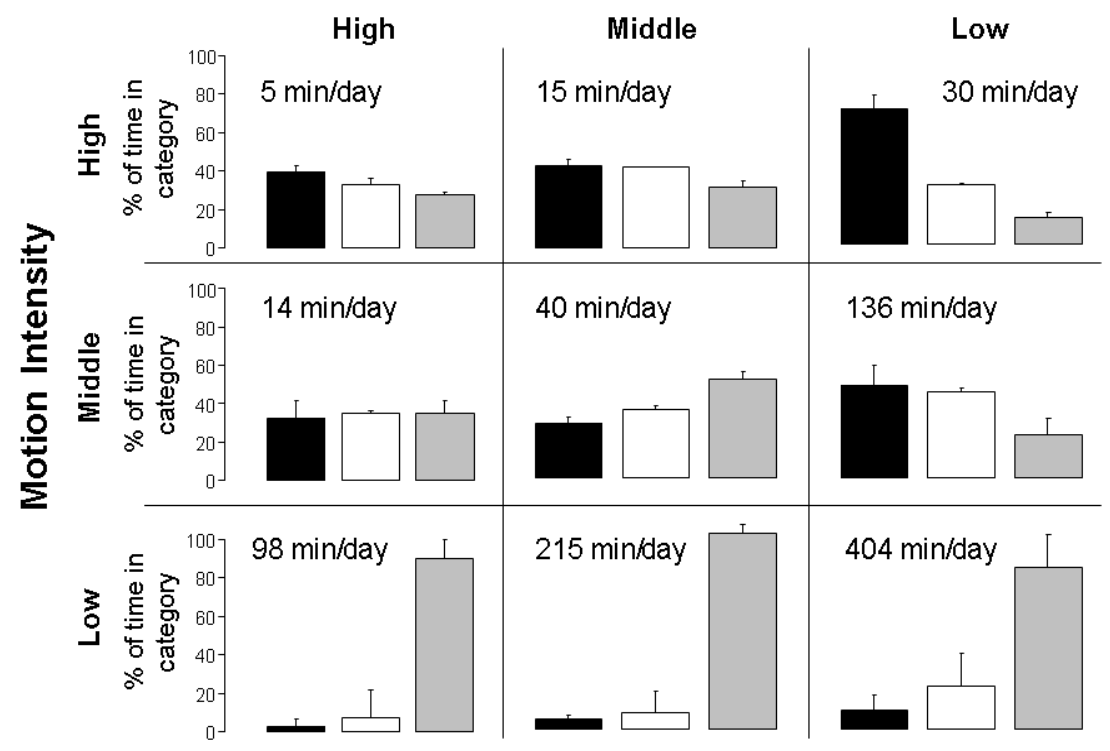

Figure 5.5: Average time spending in three levels of arm elevation and arm intensity. Bars show for each category the percentage of ambulation time (black bars), standing time (white bars) and sitting time (grey bars). 
One-second arm activities were most frequent during daily life, for a total duration of $55 \pm 5$ minutes. Longer bouts of arm ADLs were performed to a lesser extent (figure 5.6).

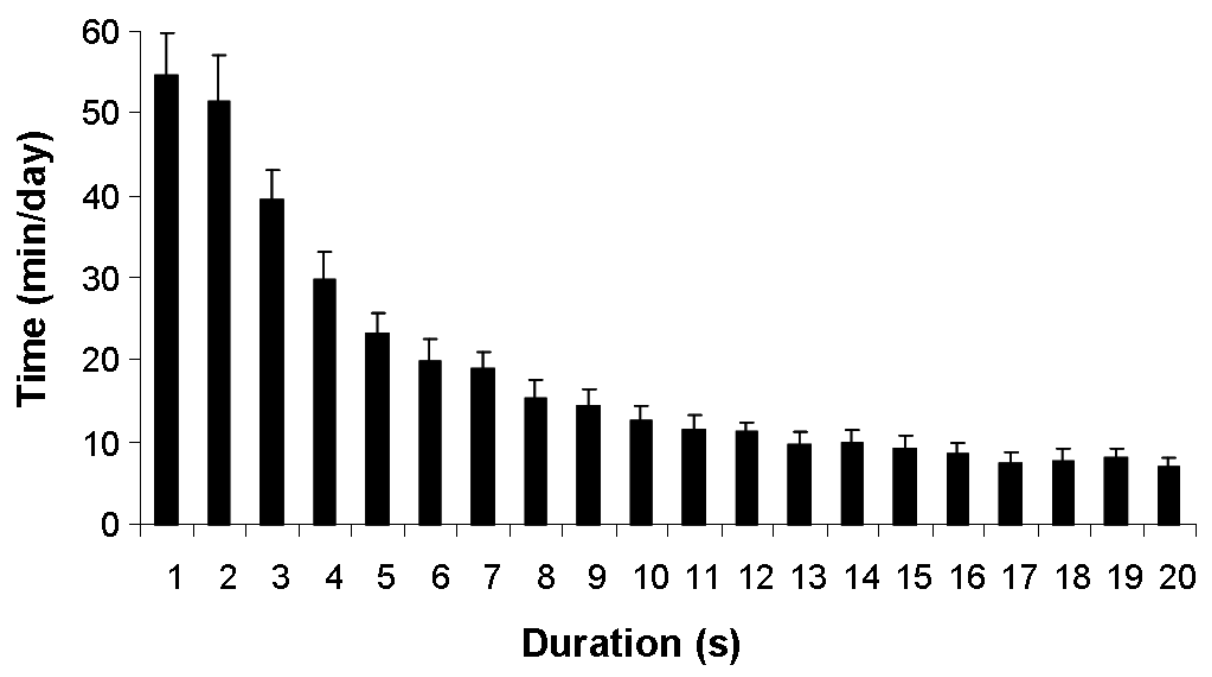

Figure 5.6: Mean $\pm S D$ time participants spent per day within a certain arm category for the duration range of $1-20$ seconds.

\section{Discussion}

To our knowledge this is the first study to assess the intensity and elevation of daily arm activities using a single tri-axial accelerometer. It was found that the elbow position resulted in highly accurate data for both outcomes (>95\%), whereas the wrist and shoulder position yielded much worse data. Moreover, it was shown that the identified arm ADL categories are all present during daily arm use in healthy subjects. Jointly, these results indicate that relevant characteristics of arm activities can be reliably and unobtrusively identified during activities of daily life. This makes the presented approach suitable for ADL monitoring in patients with limitations in arm function.

Linear accelerometers, which are commercially available, consist of usually small devices with a built in data logger which makes them unobtrusive. Moreover, they can measure for extensive periods of time. For this reason accelerometers have been used extensively outside the laboratory and clinical environment to assess the subject's daily arm use $[8,18,24,25,26]$. Accelerometers collecting data on daily arm use are typically worn on the wrist and only assess movement intensity. Two previ- 
ous studies used accelerometers to measure movement intensity and/or elevation of the arm during ADLs. For both studies the accelerometer was placed just above the elbow. Coley et al., accurately assessed arm elevation, but during motionless periods only [6]. Yet, many daily arm movements involving high elevation of the arm, are not associated with a motionless period (e.g. combing hair, dressing) [27] used multiple algorithms to calculate features representing movement intensity and elevation of the arm. Although the study provided useful information about the symmetry of the features between arms, the methods used were not yet validated to assess human arm movement intensity and elevation simultaneously. Arm movements are involved in a variety of domestic tasks (e.g. dressing, gardening, dish washing, walking). Therefore more complex and obtrusive multiple sensor systems are often used to study arm movements with a high precision $[10,11,12,13,14]$. Because less precision can be achieved using accelerometers only (e.g. accelerometers do not provide information on movements specifically within the horizontal plane) categories which define different types of movement need to be selected. For accurate assessment of arm intensity, the sensor position was not critical in this study. However, to have accurate insight in arm elevation, placement on the upper arm above the elbow is essential. The elbow sensor was most accurate presumably due to the alignment with the upper arm and distance of the sensor relative to the centre of rotation.

Short bouts of arm ADLs are highly prevalent [6]. This was confirmed by the results of Study B, indicating the importance of measuring arm activity per second. Moreover, arm ADLs in the highest elevation category are only performed for a small part of the day, which is in agreement with findings of [27]. High elevation ADLs are particularly important for maintaining independence in daily life (e.g. dress, reach). This implies the need for long-term monitoring for accurate assessment of daily arm function.

\section{Study limitations}

The classification tree used in this study to categorize arm activities was developed using data of specific arm postures and movements controlled by a marked wall and a metronome. The developed classification method was validated based on the same movements and not on a large variety of arm movements which are common in daily life. Though the latter would have been more convenient, such analyses require an expensive 3-D motion capture system and time consuming measurements to capture orientation and intensity level of the arm.

The present study evaluated daily arm use in a relatively small group of young healthy subjects. As a next step it would be interesting to acquire reference values 
of daily arm use of a more diverse healthy population. These values can then be used to evaluate functional performance of individual subjects that experience problems with performing daily life activities [28]. This enables direct and individualized feedback in the situation that matters the most (i.e. home).

Conclusions

Arm elevation and intensity are important to assess as they combined provide both quantitative and qualitative information on functional use. For accurate assessment of arm intensity, the sensor position is not critical. However, to have accurate insight in both arm elevation and intensity, placement on the upper arm above the elbow is essential. All categories of arm intensity and elevation are manifest during normal daily arm use. These results give confidence that single-unit accelerometers can provide an objective, real world index of upper-limb use.

\section{Acknowledgement}

The authors are grateful to the volunteers who participated in the present study. 


\section{References}

1. Cheung VH, Gray L, Karunanithi M (2011) Review of accelerometry for determining daily activity among elderly patients. Arch Phys Med Rehabil 92: 998-1014.

2. Katz JN, Wright EA, Baron JA, Losina E (2009) Development and validation of an index of musculoskeletal functional limitations. BMC Musculoskelet Disord 10: 62.

3. Kontaxis A, Cutti AG, Johnson GR, Veeger HE (2009) A framework for the definition of standardized protocols for measuring upper-extremity kinematics. Clin Biomech (Bristol, Avon) 24: 246-253.

4. Slobogean GP, Slobogean BL (2011) Measuring shoulder injury function: common scales and checklists. Injury 42: 248-252.

5. Uswatte G, Foo WL, Olmstead H, Lopez K, Holand A, et al. (2005) Ambulatory monitoring of arm movement using accelerometry: an objective measure of upper-extremity rehabilitation in persons with chronic stroke. Arch Phys Med Rehabil 86: 1498-1501.

6. Coley B, Jolles BM, Farron A, Aminian K (2008) Arm position during daily activity. Gait Posture 28: 581-587.

7. Schasfoort FC, Bussmann JB, Zandbergen AM, Stam HJ (2003) Impact of upper limb complex regional pain syndrome type 1 on everyday life measured with a novel upper limb-activity monitor. Pain 101: 79-88.

8. de Niet M, Bussmann JB, Ribbers GM, Stam HJ (2007) The stroke upper-limb activity monitor: its sensitivity to measure hemiplegic upper-limb activity during daily life. Arch Phys Med Rehabil 88: 1121-1126.

9. Cutti AG, Giovanardi A, Rocchi L, Davalli A, Sacchetti R (2008) Ambulatory measurement of shoulder and elbow kinematics through inertial and magnetic sensors. Med Biol Eng Comput 46: 169-178.

10. Bachmann ER, McGhee RB, Yun X, Zyda MJ (2001) Inertial and magnetic posture tracking for inserting humans into networked virtual environments. Proc ACM Symp VRST Banff, AB, Canada: 9-16.

11. de Vries WH, Veeger HE, Cutti AG, Baten C, van der Helm FC (2010) Functionally interpretable local coordinate systems for the upper extremity using inertial \& magnetic measurement systems. J Biomech 43: 1983-1988.

12. Luinge HJ, Veltink PH, Baten CT (2007) Ambulatory measurement of arm orientation. J Biomech 40: 78-85.

13. Murgia A, Kerkhofs V, Savelberg H, Meijer K (2010) A portable device for the clinical assessment of upper limb motion and muscle synergies. Conf Proc IEEE Eng Med Biol Soc: 931-934.

14. Zhou H, Hu H, Tao Y (2006) Inertial measurements of upper limb motion. Med Biol Eng Comput 44: 479-487.

15. Hyde RA, Ketteringham LP, Neild SA, Jones RS (2008) Estimation of upper-limb orientation based on accelerometer and gyroscope measurements. IEEE Trans Biomed Eng 55: 746-754.

16. de Vries WH, Veeger HE, Baten CT, van der Helm FC (2009) Magnetic distortion in motion labs, implications for validating inertial magnetic sensors. Gait Posture 29: 535-541.

17. Coley B, Jolles BM, Farron A, Aminian K (2009) Detection of the movement of the humerus during daily activity. Med Biol Eng Comput 47: 467-474.

18. Schasfoort FC, Bussmann JB, Martens WL, Stam HJ (2006) Objective measurement of upper limb activity and mobility during everyday behavior using ambulatory accelerometry: the upper limb activity monitor. Behav Res Methods 38: 439-446.

19. Zhou $\mathrm{H}$, Stone $\mathrm{T}$, Hu H, Harris N (2008) Use of multiple wearable inertial sensors in upper limb motion tracking. Med Eng Phys 30: 123-133.

20. Huang HY, Chen WC, Cheng WC, Lai YT, Lin JJ (2010) RT3 accelerometer shoulder activity: discrimination of activity levels by the RT3 accelerometer for the assessment of shoulder physical activity (SPA). Man Ther 16: 172-176. 
21. Amasay T, zodrow K, Kincl L, Hess J, Karduna A (2009) Validation of tri-axial accelerometer for the calculation of elevation angles. International Journal of Industrial Ergonomics 39: 783-789.

22. Bernmark E, Wiktorin C (2002) A triaxial accelerometer for measuring arm movements. Appl Ergon 33: 541-547.

23. Preece SJ, Goulermas JY, Kenney LPJ, Howard D, Meijer K, et al. (2009) Activity identification using body-mounted sensors-a review of classification techniques. Physiological Measurement 30: R1R33.

24. Uswatte G, Giuliani C, Winstein C, Zeringue A, Hobbs L, et al. (2006) Validity of accelerometry for monitoring real-world arm activity in patients with subacute stroke: evidence from the extremity constraint-induced therapy evaluation trial. Arch Phys Med Rehabil 87: 1340-1345.

25. Rand D, Eng JJ (2010) Arm-Hand Use in Healthy Older Adults. The American Journal of Occupational Therapy 64: 877-885.

26. Liao WW, Wu CY, Hsieh YW, Lin KC, Chang WY (2011) Effects of robot-assisted upper limb rehabilitation on daily function and real-world arm activity in patients with chronic stroke: a randomized controlled trial. Clin Rehabil 26: 111-120.

27. Acuna M, Amasay T, Karduna AR (2010) The reliability of side to side measurements of upper extremity activity levels in healthy subjects. BMC Musculoskelet Disord 11: 168.

28. Annegarn J, Meijer K, Passos VL, Stute K, Wiechert J, et al. (2012) Problematic activities of daily life are weakly associated with clinical characteristics in COPD. J Am Med Dir Assoc 13: 284-290. 



\section{Chapter 6}

\section{Characteristics of daily arm activities in patients with chronic obstructive pulmonary disease}

Janneke Annegarn, Martijn A. Spruit, Valéria Lima Passos, Hans H.C.M Savelberg, Annemie M.W.J. Schols, Emiel F.M. Wouters and Kenneth Meijer.

Submitted for publication 


\begin{abstract}
Arm activities are required for maintenance of self-care and independent living. To date, it remains unknown whether and to what extent arm activities of daily life (ADLs) in COPD patients are different compared to healthy elderly peers and the extent to which they perform arm ADLs at a higher proportion of the maximum upper limb muscle effort. Daily arm and leg activities were assessed in the home environment in 21 COPD patients and 24 healthy elderly (part 1); and relative efforts of the trapezius, deltoid and biceps muscles were studied using electromyography during domestic arm ADLs in a laboratory setting in 17 COPD patients and 15 healthy (part 2). In both parts arm and leg activities were measured by two accelerometers (worn on the arm and leg). After correction for walking time, the time spent on arm ADLs was similar between patients with COPD and healthy subjects $(p=0.52)$, while the intensity of arm activities was lower in patients with COPD $(p=0.041)$. In the laboratory setting, a majority of the arm ADLs were performed at a lower intensity by COPD patients, while the trapezius muscle effort was significantly higher during several domestic arm ADLs compared to healthy elderly subjects. Patients with COPD have similar duration of arm ADLs compared to healthy subjects after correction for walking time, but they perform arm activities at a lower intensity. Moreover, patients perform some daily arm activities at a higher proportion of maximum muscle effort.
\end{abstract}




\section{Introduction}

Physical inactivity is a major risk factor for accelerated disability [1], and a worse prognosis in patients with chronic obstructive pulmonary disease (COPD) [2,3]. Indeed, regular physical activities like walking are problematic in patients with COPD $[4,5]$. Moreover, simple domestic activities of daily living (ADLs) that involve arms, like cleaning and dressing, can also be impaired and symptom-inducing $[5,6]$. These impairments are of concern because they limit a patients' independence during everyday life $[7,8]$.

To date, characteristics of arm ADLs in COPD are lacking in the literature. Patients with COPD experience a relatively high metabolic load and dyspnea during the performance of self-paced domestic arm ADLs compared to healthy elderly [6]. Moreover, arm elevation in COPD patients has been associated with loss of vital capacity $[9,10,11,12,13,14,15,16]$. Therefore arm activities, especially when involving arm elevation are demanding for muscles with an inspiratory function, like the trapezius muscle [17]. Consequently, patients with COPD may reduce arm ADLs to reduce the burden of task-related dyspnoea. Then again, a preservation of deltoid and biceps muscle endurance has been reported in patients with COPD, which conceivably can be explained by maintenance of arm ADLs [18,19,20,21].

Assessment of arm activities in COPD patients' home environment is essential to better understand the problematic daily arm activities. This requires a validated arm accelerometer [22] and detailed electromyographic (EMG) evaluation of muscle function during daily arm activities. Therefore, the present study aimed to quantify daily arm activities in patients with COPD and healthy elderly subjects. Moreover, muscle effort was assessed of the trapezius, biceps and deltoid muscles during the performance of daily arm activities in a laboratory setting to explore the mechanisms underlying the decreased arm activity in COPD. It was hypothesized that daily arm activities were preserved as they are still required for maintenance of self-care and independent living and that this comes at the expense of a larger muscle effort.

\section{Methods}

\section{Study description}

This study consisted of two parts. Part 1 was done to assess arm and leg activity in daily life in participants' home environment. Part 2 was conducted to study relative effort of the trapezius, deltoid and biceps muscles during daily arm activities in a 
laboratory setting. In both parts arm and leg activities were measured by two accelerometers (worn on the upper arm just above the elbow and upper leg just above the knee). Activities of the legs (e.g. walking) result in whole body movement including the arms. To compare activities of the arms only (no leg movements involved) between COPD and healthy subjects, arm activities were not counted when leg activity was measured simultaneously (e.g. arm activities without leg activity).

\section{Patient selection}

All patients were recruited at $\mathrm{ClRO}+$, a center of expertise for chronic organ failure in Horn, the Netherlands [23]. Inclusion criteria were: 1) no exacerbation in past 4 weeks; and 2) absence of pathologic conditions that could impair physical activities in daily life, like stroke. Healthy subjects were relatives of employees and students of $\mathrm{CIRO}+$ or healthy spouses of patients at CIRO+. Inclusion criteria were the same as the COPD group, with the addition of normal spirometry results and no use of physician-prescribed drugs. Of the subjects included, five COPD patients and 15 healthy subjects participated in both parts. The study was approved by the ethical committee of Maastricht University Medical Centre (Part 1: MEC 10-3-086; part 2: MEC 10-3-077). All participants gave written informed consent prior to inclusion.

\section{Instrumentation}

Two triaxial accelerometers (CAM, Maastricht Instruments B.V., Maastricht, the Netherlands) were used in both parts of the study to accurately assess activities and postures. The CAM was used as it can assess both leg activity (CAM leg) [24] and arm activity $\left(\mathrm{CAM}_{\mathrm{arm}}\right)$ [22]. In brief, $\mathrm{CAM}_{\text {leg }}$ measures the time spent walking (or other dynamic activities), weight bearing postures (standing) and non-weight bearing postures (sitting and lying), as well as movement intensity during walking. CAM arm $_{\text {m }}$ measures the intensity and elevation of the arm at each second, which will then be categorized into three levels of movement intensity (posture: holding still, medium: moving slow, high: moving fast) and arm elevation (low: $\leq 45^{\circ}$, medium: $45-90^{\circ}$, high: $>90^{\circ}$, figure 6.1 ). 


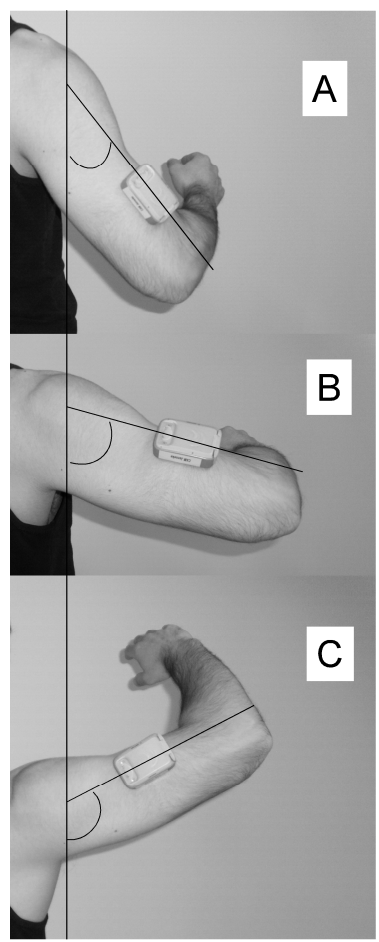

Figure 6.1: Subject holding the arm still at different arm elevation levels (low: $\leq 45^{\circ}(\mathrm{A})$, medium: $45-90^{\circ}$ (B), high: $\left.>90^{\circ}(\mathrm{C})\right)$. The CAM was attached to the arm.

Pulmonary function [25], the degree of dyspnea and fatigue [6], muscle strength and endurance of the quadriceps muscle and biceps muscle using a dynamometer [26] (only part 1) and maximal and taskrelated effort of the trapezius muscle, biceps muscle and deltoid muscle [27] (only part 2) were assessed.

\section{Pulmonary function}

For all participants post bronchodilator forced expiratory volume in the first second (FEV1) and forced vital capacity were determined using spirometry (Master Screen PFT Pro, CareFusion, Houten, the Netherlands) according to the guidelines of the European Respiratory Society [25].

\section{Borg symptom scores}

Participants were asked to score the degree of dyspnea and fatigue using a modified Borg symptom score ranging from 0 (no symptoms) to 10 points (worst symptoms) at different time points [6]. Borg scores for part 1 of the study were obtained at three time points: 1 ) right before the strength test, 2) right before the endurance test, 3) directly after the endurance test (flow chart figure 6.2A). Borg scores for part 1 of the study were obtained before and after the protocol (flow chart figure $6.2 B)$. 
A

B

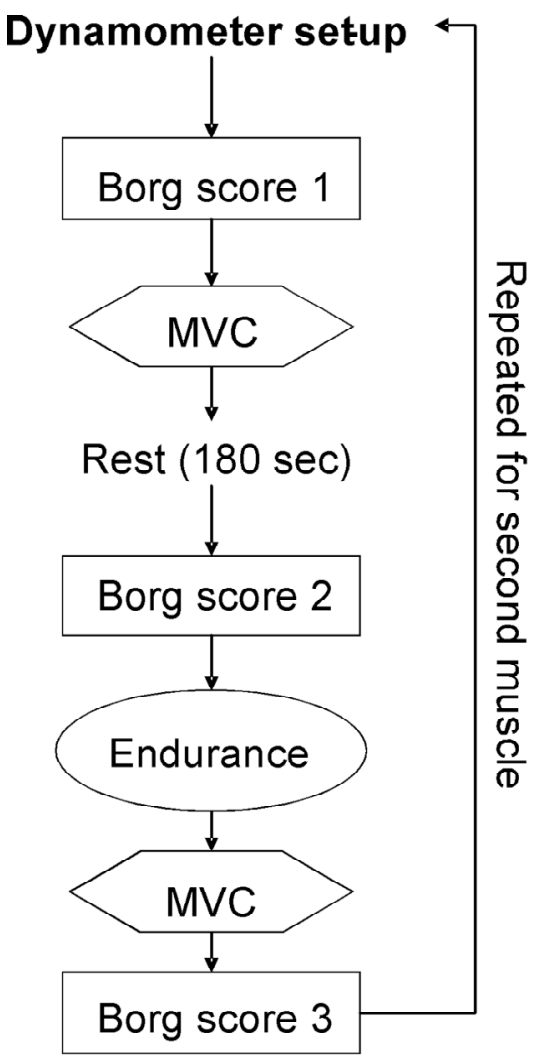

Sensor placement

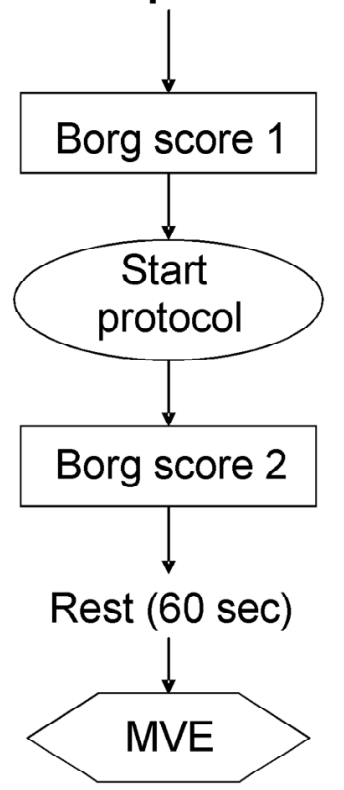

Figure 6.2: Flow charts. A: muscle strength and endurance testing for quadriceps muscle of the lower extremity and the biceps muscle of the upper extremity. B: protocol for the assessment of daily arm activities in the laboratory setting.

\section{Strength and endurance testing}

Strength and endurance of the quadriceps muscle and biceps muscle were evaluated on a dynamometer (Biodex system 3, Biodex corporation, Shirley, New York, U.S.) using an adaptation of a protocol previously described by Allaire et al. [26]. A flow chart of strength and endurance testing is provided in figure 6.2A.

\section{Dynamometer set-up}

All muscle function experiments started with establishment of the correct seating position. For measurement of the QF the seating position was adjusted to ensure that the lateral epicondyle of the right femur was centred with the axis of rotation of the lever arm. The hip and knee angle were adjusted to $90^{\circ}$. The lower leg was 
firmly attached to the lever arm with a padded Velcro strap. For measurement of the $\mathrm{BB}$ the right shoulder was adjusted to $30^{\circ}$ exorotation, with an elbow angle of $90^{\circ}$. The wrist was firmly attached to the lever arm with a padded Velcro strap. To prevent for extraneous movement during the isometric contractions, straps were secured across the waist and shoulders.

\section{Muscle strength testing}

In order to determine the maximal voluntary contraction (MVC), subjects performed three maximal contractions, each lasting three seconds and separated by 120 seconds rest. A countdown was given, followed by strong verbal encouragement to maximize torque. In case of an MVC variability of $>10 \%$ we asked participants to perform a fourth maneuver. All participants were able to produce three reproducible maximal contractions in their first three or four attempts. The highest value of the contractions was considered as the MVC.

\section{Muscle endurance testing}

After three minutes of rest following the MVC manoeuvres, subjects were instructed to maintain an isometric force $\mathrm{n}$ representing $60 \%$ of their MVC until exhaustion. A computer screen served as a feedback mechanism to help subjects maintain the determined submaximal tension. Subjects were strongly encouraged to pursue until tension drop to $50 \%$ of MVC. Peripheral muscle endurance was thus assessed by the time to fatigue, defined as the time at which the isometric contraction reached $50 \%$ MVC. Mean torque $(\mathrm{Nm})$ and the coefficient of variation $(\mathrm{CV})$ of the torque (\%) during the endurance test were similar between COPD patients and healthy subjects for the quadriceps muscle (COPD mean torque: $62.2 \pm 2.8 \mathrm{Nm}$, healthy mean torque: $61.2 \pm 1.7 \mathrm{Nm}$, COPD CV: $6.4 \pm 2.1 \%$, healthy CV: $6.2 \pm 2.1 \%)$ and for the biceps muscle (COPD mean torque: $63.0 \pm 3.7 \mathrm{Nm}$, healthy mean torque: $62.1 \pm 3.4 \mathrm{Nm}$, COPD CV: $2.9 \pm 1.0 \%$, healthy CV: $2.7 \pm 0.9 \%)$. An example of toque output during the endurance test of a random COPD patient is shown in figure 6.3. 


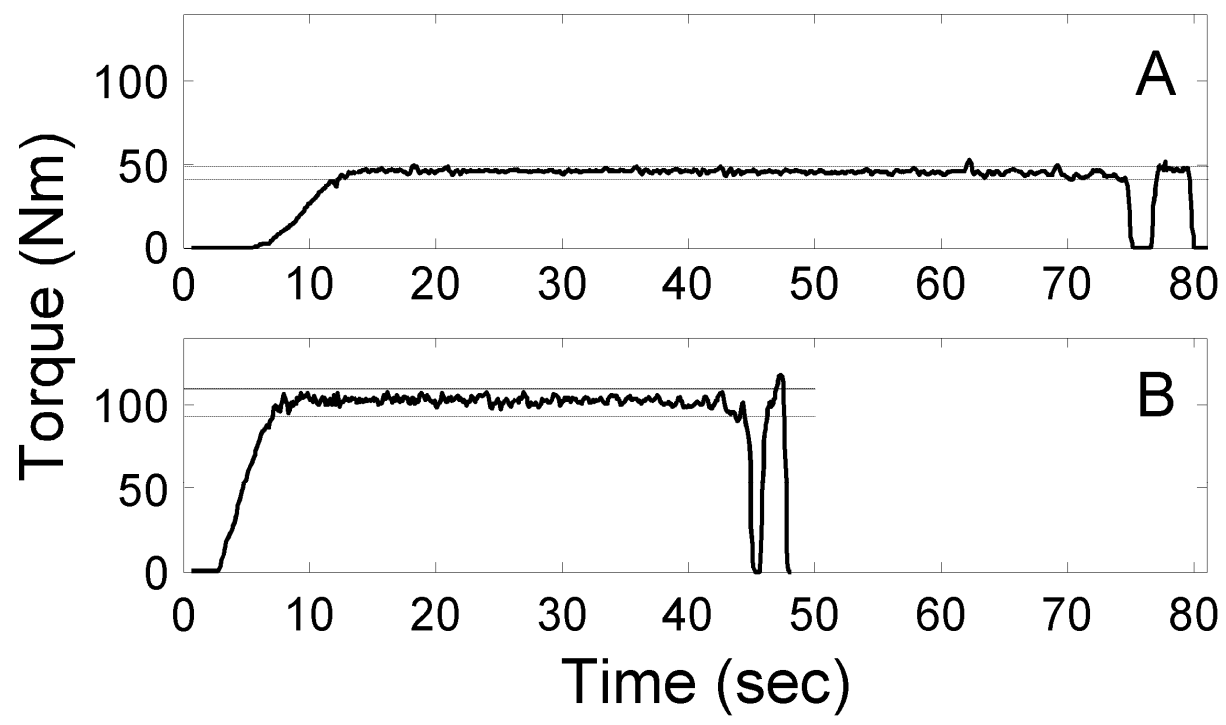

Figure 6.3: Example of torque output during the endurance test of the biceps muscle $(A)$ and the quadriceps muscle (B). The subject is a COPD patient.

\section{Relative muscle effort}

Relative muscle effort was measured in a controlled setting during a standardized protocol. For this purpose electromyography (EMG) surface was recorded from the using a Programmable Ambulant Signal AcQuisition - system (PASAQ). A common ground electrode was placed on the ulnar styloid process. The cables from the electrodes were taped to the skin and placed into the PASAQ which was worn in a small backpack on the back of the participant (figure 6.4). The signal from the electrodes was sampled at $1000 \mathrm{~Hz}$. MATLAB(c) software and algorithms were used to calculate the muscle effort as described previously [27]. For this purpose signals were rectified, smoothed at a time constant of 0.01 seconds and down-sampled by a factor of 100. A value representing muscle effort was obtained for each second by averaging over 10 samples. To obtain the relative muscle effort these values were normalized for each subject in such way they expressed the percentage of the participant's maximum voluntary effort assessed after the protocol (flow chart figure 6.2B). 


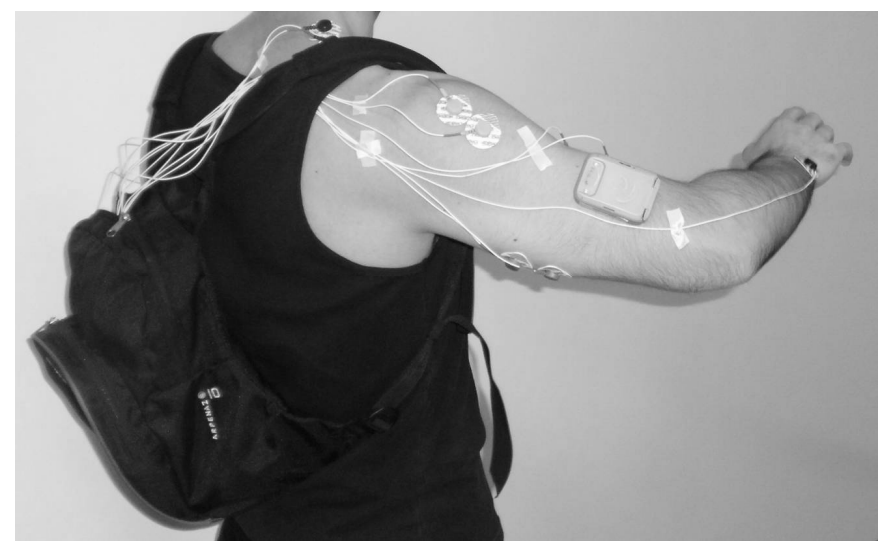

Figure 6.4: Subject equipped with sensors for the assessment of daily arm activities in the laboratory setting. The cables from the EMG electrodes were taped to the skin and placed into the PASAQ which was worn in a small backpack on the back of the participant. The CAM was attached above the elbow.

\section{Maximum voluntary effort}

For this purpose participants were asked for a maximal voluntary effort for three seconds for each muscle twice. Between efforts 30 seconds of rest was taken. For the trapezius muscle participants were asked sit straight, to grasp the chair seat and pull as hard as possible toward the ceiling (figure 6.5, position 1 ). For the biceps muscle participants were asked to sit in front of a table fixed to the wall, and push with the wrist against the table and push as hard as possible toward the ceiling (figure 6.5, position 2). For the deltoid muscle participants were asked to sit with their elbow against the table and push as hard as possible toward the wall (figure 6.5 , position 3). 


\section{Trapezius}

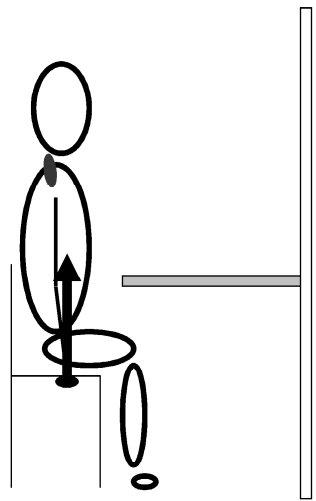

Side view Position 1
Biceps

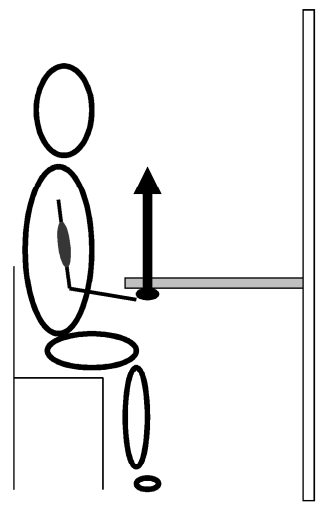

Side view Position 2
Deltoid

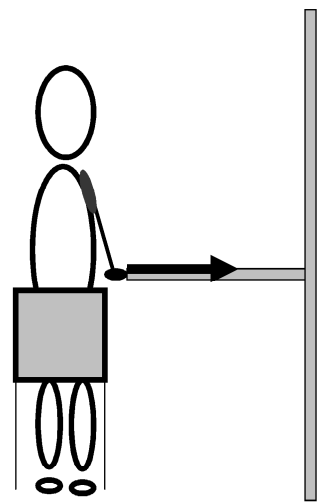

Back view Position 3

Figure 6.5: Positions in which maximal voluntary effort of the trapezius, biceps and deltoid muscle were performed. The large arrows represent the direction of force.

\section{Part 1: Assessment in daily life}

Participants were asked to wear the $\mathrm{CAM}_{\mathrm{arm}}$ and $\mathrm{CAM}_{\mathrm{leg}}$ simultaneously for six full days during waking hours. The CAM is not water proof, so subjects had to remove the CAMs while showering or swimming. Both sensors were programmed to record automatically from 07.00 AM to 07.00 PM. Evening hours (after 07.00 PM) were not included in the analysis because it is likely that elderly people in general have considerably fewer activities during this period when compared with the other periods of the day [28]. Mean wearing time was similar between patients with COPD and healthy elderly subjects $(5.3 \pm 0.8$ days for $9.9 \pm 1.2$ hours; and $5.6 \pm 0.7$ days for $10.4 \pm 0.9$ hours, respectively). Since the subjects were not all measured for exactly the same duration (for example due to the fact that some patients woke up after 07.00 AM), time spent in different activity categories were normalized for 10 hours per day and averaged over the amount of wearing days.

\section{Part 2: Assessment in laboratory setting}

Participants performed 12 domestic activities of daily life at the Department of Occupational Therapy in CIRO+ (figure 6.6). The activities were performed in the same order by all participants and they were asked to carry out activities as they would do during daily life, i.e. at their preferred intensity and elevation of the arm. Each activity lasted for 30 seconds and was followed by 30 to 60 seconds rest. Time 
was kept using a stopwatch. Five seconds in the beginning and end of each activity were excluded for further analyses. During the protocol arm elevation, arm intensity and effort of the biceps, deltoid and trapezius muscles were assessed using EMG and expressed as \% of maximal muscle effort.

The data in figure 6.6 is obtained from a random COPD patient. Note that the patient was sitting during rest after the activities 'drink water', 'stretching arms', 'shaking hands', 'draw picture', 'fold towels' and 'face care'. In rest the patient was leaning on the table or walking aid, showing an increased arm elevation. The first activity (cleaning the window) is a combination of high intensity and high elevation of the arm, resulting in the highest relative muscle effort within this patient. The second activity (writing on board) shows the effect of high elevation in combination with a low intensity of the arm on relative muscle effort. The third activity (cleaning the sink) shows the effect of low elevation in combination with a high intensity of the arm on relative muscle effort. 


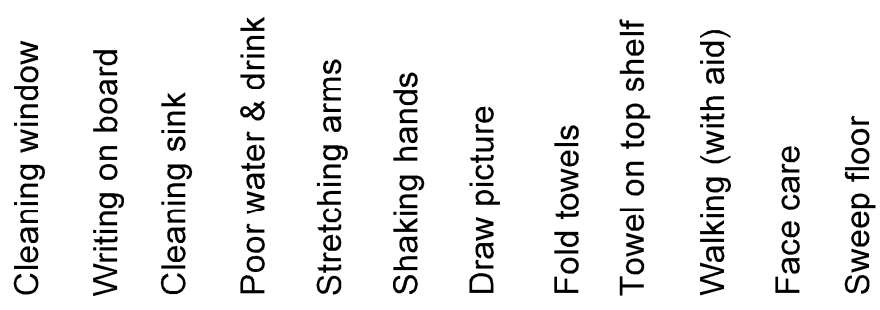
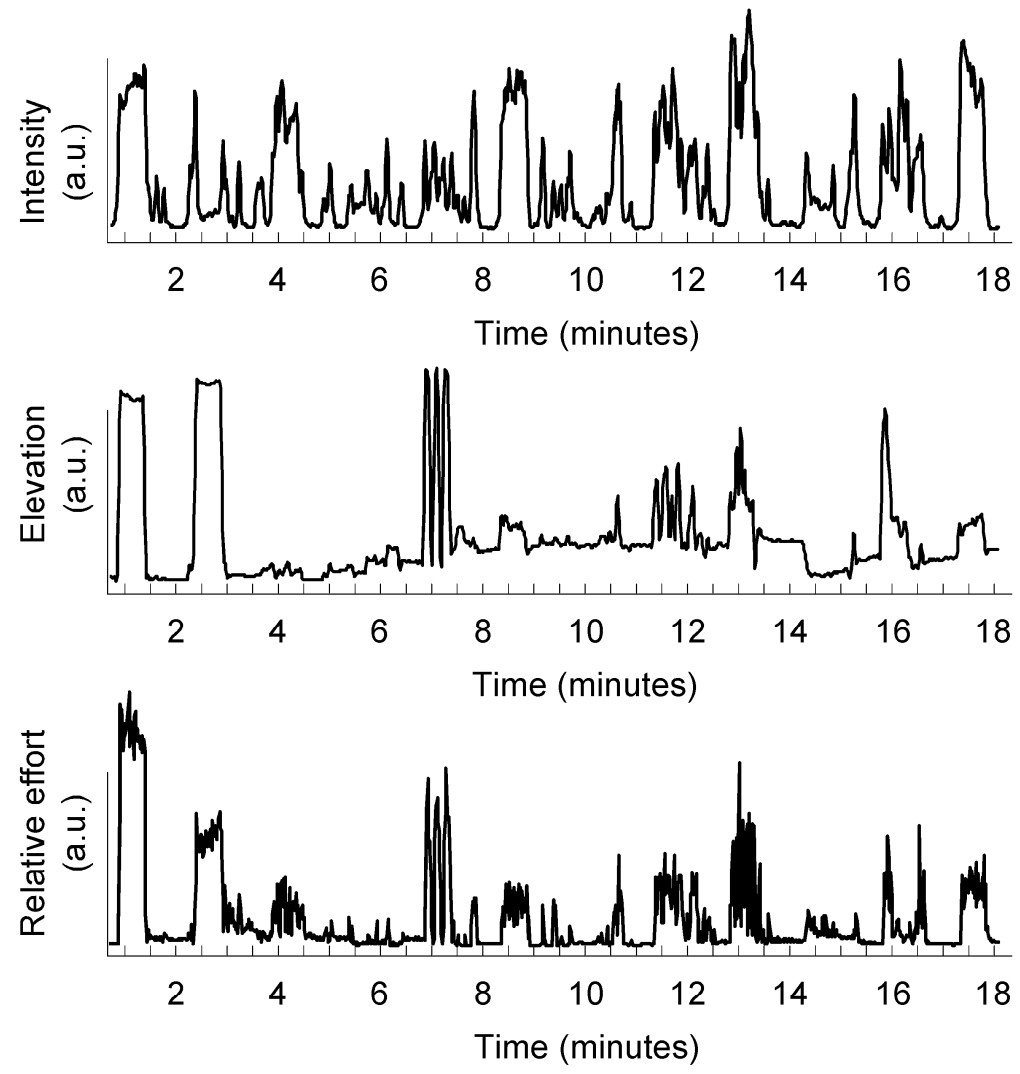

Figure 6.6: Example of arm intensity, arm elevation and relative effort of the deltoid muscle obtained during the protocol in a controlled setting.

Abbreviations; a.u.: arbitrary unit. 


\section{Statistical analysis}

Continuous and categorical variables are presented as means \pm SD or proportion, respectively. Relative muscle effort of all three muscles were displaying skewness to the right in their frequency distributions and were therefore $\log (10)$ transformed. Data on COPD and healthy patients were compared by using independent t-test or Chi-square test, when appropriate. Arm movement intensity can be influenced by leg activity. Therefore, data were re-analyzed after excluding walking time.

Specific in part 2, variables (e.g. muscle effort, intensity and elevation of the arm) for each activity in the protocol were first averaged over the repeated measures (20 samples per activity per subject) and then compared between groups using independent t-test. In addition, all relative muscle effort data was compared between groups, intensity and elevation levels of the arm. To account for the nested structure of the data (repeated measures within subjects over different controlled conditions), a random intercept model analysis was conducted. Subjects were the random factor. Gender, group (COPD versus healthy), arm intensity (holding still versus moving slow versus moving fast) and arm elevation (low versus medium versus high) were the fixed effects.

A priori, the level of significance was set at $p<0.05$. Data analysis was performed using the Statistical Package for Social Science (SPSS; Chicago, IL), version 15.0. 


\section{Results part 1}

\section{Subjects' characteristics}

Daily arm and leg activities were assessed in 21 COPD patients and 24 healthy elderly subjects. Patients were suffering from mild to very severe COPD [29]. Gender distribution, age, weight and height were similar between groups. COPD patients had lower quadriceps muscle strength and endurance, while no significant differences were found for the biceps muscle (table 6.1). The degree of fatigue and dyspnea are described in figure 6.7.

Table 6.1: Patients' characteristics (part 1)

\begin{tabular}{|c|c|c|c|c|}
\hline & $\begin{array}{l}\text { COPD } \\
(n=21)\end{array}$ & $\begin{array}{l}\text { Healthy } \\
(n=24)\end{array}$ & $95 \% \mathrm{Cl}$ & P-values \\
\hline$\%$ men & 76.2 & 70.8 & $-0.3-0.2$ & 0.746 \\
\hline Age (years) & $64.1 \pm 7.7$ & $62.4 \pm 6.2$ & $-2.5-5.9$ & 0.41 \\
\hline Weight (kg) & $79.2 \pm 14.5$ & $77.3 \pm 12.8$ & $-6.2-10.2$ & 0.631 \\
\hline Height (m) & $1.73 \pm 0.08$ & $1.74 \pm 0.09$ & $-0.06-0.04$ & 0.682 \\
\hline BMI $\left(\mathrm{kg} / \mathrm{m}^{2}\right)$ & $26.5 \pm 4.9$ & $25.4 \pm 3.3$ & $-1.4-3.6$ & 0.379 \\
\hline FEV1 (L) & $1.73 \pm 0.66$ & $3.53 \pm 0.54$ & $-2.2--1.4$ & $<0.001$ \\
\hline FEV1 (\% predicted) & $57.8 \pm 18.9$ & $117.6 \pm 19.2$ & $-71.3--48.4$ & $<0.001$ \\
\hline FVC (L) & $3.76 \pm 0.92$ & $4.42 \pm 0.50$ & $-1.11--0.19$ & 0.007 \\
\hline FVC (\% predicted) & $99.6 \pm 17.3$ & $118.6 \pm 23.4$ & $-31.6--6.6$ & 0.004 \\
\hline Tiffeneau index (\%) & $45.8 \pm 13.5$ & $79.9 \pm 5.9$ & $-40.6--27.6$ & $<0.001$ \\
\hline QF strength (Nm) & $104 \pm 37$ & $134 \pm 39$ & $-52--7$ & 0.012 \\
\hline QF endurance (sec) & $38 \pm 14$ & $49 \pm 12$ & $-19--4$ & 0.005 \\
\hline BB strength (Nm) & $49 \pm 15$ & $57 \pm 15$ & $-18-0$ & 0.056 \\
\hline BB endurance (sec) & $60 \pm 46$ & $76 \pm 28$ & $-38-7$ & 0.17 \\
\hline
\end{tabular}

Value expressed as mean \pm standard deviation (SD)

Abbreviations; $\mathrm{Cl}$ : Confidence Interval of the mean difference, QF: Quadriceps femoris muscle, BB: Biceps Brachii muscle 

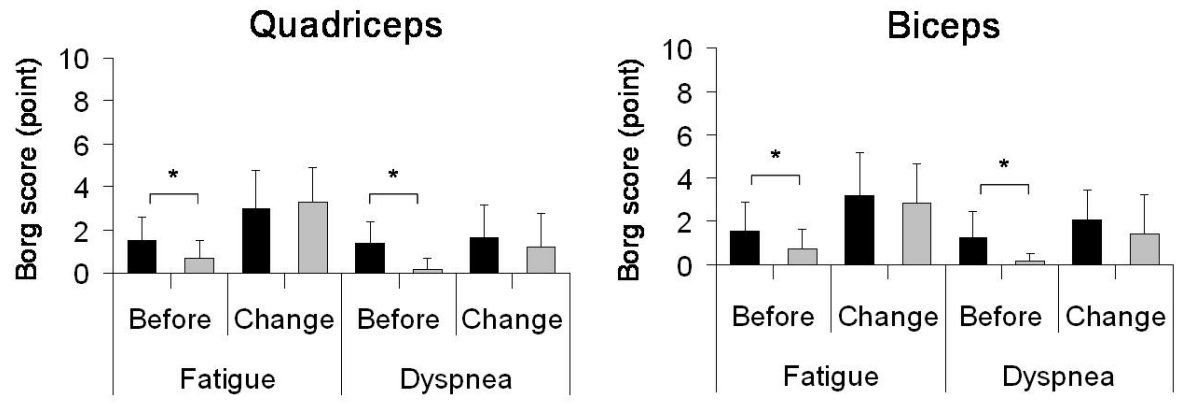

Figure 6.7: Mean \pm SD of borg scores for fatigue and dyspnea at the beginning of the endurance test and the change in scores after the endurance test for the quadriceps and biceps muscles. Scores are presented for COPD patients and healthy subjects. *: $p<0.05$.

\section{Daily leg activity and postures}

COPD patients spent more time sitting and/or lying (COPD: $394 \pm 66$ minutes, healthy: $328 \pm 77$ minutes, $p<0.01$ ) and less time walking (COPD: $86 \pm 39$ minutes, healthy: $134 \pm 53$ minutes, $p<0.01$ ). Time spent in standing was similar between groups (COPD: $120 \pm 38$ minutes, healthy: $136 \pm 49$ minutes, $p=0.11$ ) (figure 6.8). Moreover, intensity of walking was lower in COPD patients (COPD: $38.2 \pm 3.7$ a.u., healthy: $47.3 \pm 7.7$ a.u., $p<0.01$ ).

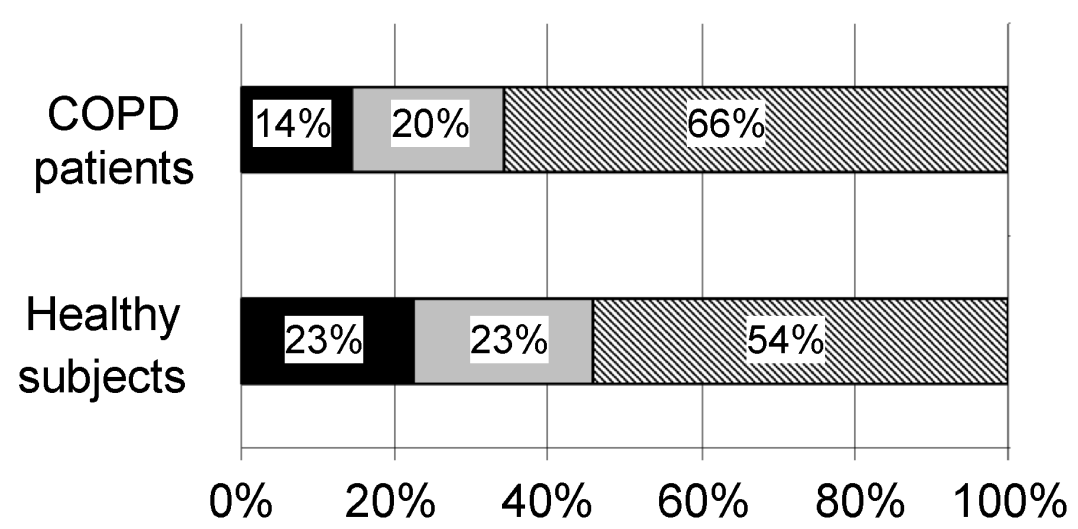

Figure 6.8: Bars represent percentage of time spent on walking (black), standing (light grey) and sitting (dark grey). 


\section{Daily arm activity and postures}

COPD patients spent more time on arm postures and less time on arm activities compared to healthy subjects. Differences in arm activity time were only present during low arm elevation, which is the typical arm category during walking. After correction for walking time, the time spent on daily arm activities was similar between patients with COPD and healthy elderly subjects. Nevertheless, the intensity of arm activities was lower in patients with COPD ( $p=0.002)$, also after correction of leg activity time $(p=0.041)$ (table 6.2).

Table 6.2: Characteristics of daily arm activities

\begin{tabular}{|c|c|c|c|c|c|c|c|}
\hline & & \multicolumn{3}{|c|}{ Total period } & \multicolumn{3}{|c|}{ Without walking } \\
\hline & & COPD & Healthy & $\begin{array}{c}95 \% \mathrm{Cl} \\
{[P \text { values }]}\end{array}$ & COPD & Healthy & $\begin{array}{c}95 \% \mathrm{Cl} \\
{[P \text { values }]}\end{array}$ \\
\hline \multicolumn{2}{|c|}{ Arm postures (min) } & $451.4 \pm 56.9$ & $400.1 \pm 62$ & $\begin{array}{c}15.3-87.2 \\
{[0.006]}\end{array}$ & $436.1 \pm 56.6$ & $382.3 \pm 65.5$ & $\begin{array}{c}16.8-90.9 \\
{[0.005]}\end{array}$ \\
\hline \multicolumn{2}{|c|}{ Arm activity (min) } & $148.6 \pm 56.9$ & $199.9 \pm 62$ & $\begin{array}{c}-87.2--15.3 \\
{[0.006]}\end{array}$ & $77.2 \pm 28.2$ & $82.6 \pm 28.4$ & $\begin{array}{c}-22.5-11.6 \\
{[0.524]}\end{array}$ \\
\hline Intensity & Elevation & \multicolumn{6}{|c|}{ Arm activity per category (min) } \\
\hline \multirow{3}{*}{ High } & High & $0.6 \pm 0.5$ & $0.6 \pm 0.5$ & $\begin{array}{c}-0.3-0.3 \\
{[0.847]}\end{array}$ & $0.4 \pm 0.4$ & $0.4 \pm 0.3$ & $\begin{array}{c}-0.2-0.2 \\
{[0.719]}\end{array}$ \\
\hline & Med & $7.9 \pm 3.9$ & $11.1 \pm 8.3$ & $\begin{array}{c}-7.2-0.7 \\
{[0.107]}\end{array}$ & $4.7 \pm 2.1$ & $5.5 \pm 3$ & $\begin{array}{c}-2.4-0.8 \\
{[0.302]}\end{array}$ \\
\hline & Low & $8.1 \pm 5.2$ & $22.6 \pm 23.4$ & $\begin{array}{c}-25.1--4.0 \\
{[0.007]}\end{array}$ & $3.4 \pm 2.2$ & $5 \pm 3.6$ & $\begin{array}{c}-3.4-0.2 \\
{[0.072]}\end{array}$ \\
\hline \multirow{3}{*}{ Med } & High & $2.2 \pm 1.7$ & $1.6 \pm 0.7$ & $\begin{array}{c}-0.2-1.3 \\
{[0.132]}\end{array}$ & $1.9 \pm 1.5$ & $1.3 \pm 0.5$ & $\begin{array}{c}-0.1-1.3 \\
{[0.095]}\end{array}$ \\
\hline & Med & $30.6 \pm 15.1$ & $33.5 \pm 12.2$ & $\begin{array}{c}-11.1-5.3 \\
{[0.479]}\end{array}$ & $22.4 \pm 9.7$ & $22.9 \pm 8.3$ & $\begin{array}{c}-5.9-4.9 \\
{[0.858]}\end{array}$ \\
\hline & Low & $99.3 \pm 42.2$ & $130.4 \pm 39.2$ & $\begin{array}{c}-55.5--6.6 \\
{[0.014]}\end{array}$ & $44.4 \pm 17.5$ & $47.5 \pm 17.2$ & $\begin{array}{c}-13.6-7.3 \\
{[0.546]}\end{array}$ \\
\hline \multicolumn{2}{|c|}{ Activity intensity (a.u.) } & $30.5 \pm 2.5$ & $33.6 \pm 3.6$ & $\begin{array}{c}-4.9--1.2 \\
{[0.002]}\end{array}$ & $28.5 \pm 1.7$ & $29.8 \pm 2.4$ & $\begin{array}{c}-2.6--0.1 \\
{[0.041]}\end{array}$ \\
\hline
\end{tabular}

Values expressed as mean \pm standard deviation (SD).

Abbreviations; $\mathrm{Cl}$ : Confidence Interval of the mean difference.

Note: Low intensity is considered as posture and therefore not included in the table as subcategory of arm activities. 


\section{Results part 2}

\section{Subjects' characteristics}

Relative muscle effort during domestic daily arm activities was studied in 17 COPD patients and 15 healthy elderly subjects. Gender distribution, age, weight and height were not significantly different between groups (table 6.3). A representative example of analyzed EMG and acceleration signals obtained from a COPD patient during the protocol is presented in figure 6.6. In a few cases EMG data of the trapezius muscle (COPD, $n=2$ ) and biceps muscle (COPD, $n=3$; healthy, $n=1$ ) were excluded due to loss of electrode contact and movement artifacts. The degree of fatigue and dyspnea are described in figure 6.9.

Table 6.3: Patients' characteristics (part 2)

\begin{tabular}{lllll}
\hline & $\begin{array}{l}\text { COPD } \\
(\mathrm{n}=18)\end{array}$ & $\begin{array}{l}\text { Healthy } \\
(\mathrm{n}=15)\end{array}$ & $95 \% \mathrm{Cl}$ & P value \\
\hline \% men & 55.6 & 53.3 & -0.7 & 0.588 \\
Age (years) & $62.4 \pm 8.1$ & $65 \pm 7$ & $-7.8-2.9$ & 0.351 \\
Weight (kg) & $73.7 \pm 12.9$ & $74.5 \pm 12.0$ & $-8.7-9.1$ & 0.968 \\
Height (m) & $1.70 \pm 0.09$ & $1.74 \pm 0.09$ & $-0.11-0.02$ & 0.213 \\
BMI (kg/m $\left.{ }^{2}\right)$ & $25.5 \pm 4.5$ & $24.1 \pm 2.9$ & $-1.37-4.14$ & 0.313 \\
FEV1 (L) & $1.41 \pm 0.65$ & $3.48 \pm 0.58$ & $-2.51--1.63$ & $<0.001$ \\
FEV1 (\% predicted) & $50.1 \pm 20.1$ & $118.9 \pm 16.0$ & $-81.8--55.7$ & $<0.001$ \\
FVC (L) & $3.40 \pm 0.90$ & $4.39 \pm 0.60$ & $-1.55--0.44$ & 0.001 \\
FVC (\% predicted) & $96.0 \pm 16.3$ & $124.7 \pm 24.8$ & $-43.4--14.0$ & $<0.001$ \\
Tiffeneau index (\%) & $42.3 \pm 15.2$ & $79.2 \pm 4.9$ & $-45.3--28.5$ & $<0.001$ \\
\hline
\end{tabular}

Values expressed as mean \pm standard deviation (SD).

Abbreviation; $\mathrm{Cl}$ : Confidence Interval of the mean difference. 


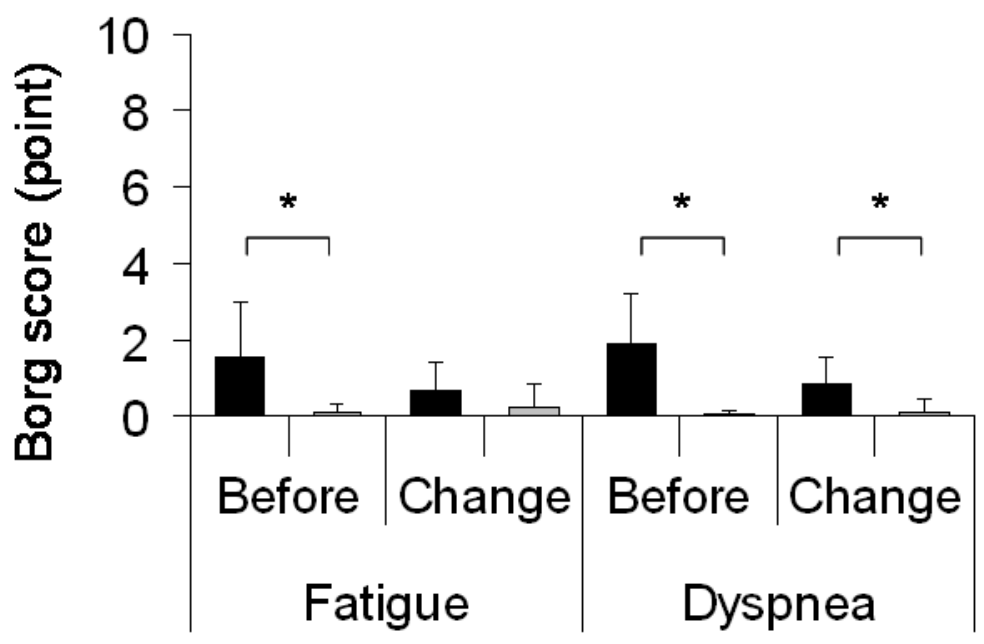

Figure 6.9: Mean \pm SD of borg scores for fatigue and dyspnea at the beginning of the protocol for assessment of daily arm activities in the laboratory setting the change in scores after the protocol. Scores are presented for COPD patients and healthy subjects. *: $p<0.05$.

COPD patients performed seven of the twelve activities of the protocol at a lower movement intensity compared to healthy subjects, while the degree of arm elevation was similar between groups. Five activities of the trapezius muscle ('Write on board', 'Shake hands', 'Put towels on the shelf', 'Walk at preferred speed', and 'Sweep floor') and one activity of the biceps muscle ('Put towels on the top shelf') were performed at a higher proportion of the maximum muscle effort compared to healthy elderly subjects. No differences were observed for the deltoid muscle. Results for each activity are presented in tables 6.4-6.5.

Activities were categorized to compare relative muscle effort between groups, intensity levels and elevation levels of the arm (figure 6.10, tables 6.6-6.7). Time spent in the high intensity categories was lower for COPD patients (table 6.8). Significant three-way interactions between group, elevation and intensity were detected, indicating that differences between COPD patients and healthy subjects depended on the levels of both intensity and elevation of the arm. Lower intensity and lower arm elevation was accompanied with lower relative effort for all muscles in both groups. The group effects (COPD versus healthy) were not the same for all muscles. For each level of orientation and intensity COPD patients clearly showed higher effort of the trapezius muscle, while no consistent differences could be found for the biceps and deltoid muscle. 
Table 6.4: mean and differences between COPD and healthy in arm intensity, arm orientation and leg intensity for each activity.

\begin{tabular}{|c|c|c|c|c|c|c|}
\hline & \multicolumn{2}{|c|}{$\begin{array}{l}\text { Arm intensity } \\
\text { (a.u.) }\end{array}$} & \multicolumn{2}{|c|}{$\begin{array}{l}\text { Arm elevation } \\
\text { (a.u.) }\end{array}$} & \multicolumn{2}{|c|}{$\begin{array}{l}\text { Leg intensity } \\
\text { (a.u.) }\end{array}$} \\
\hline & COPD & Healthy & COPD & Healthy & COPD & Healthy \\
\hline Clean window (upper part) & 61.1 & $84.4^{*}$ & -9.2 & -10 & 8.1 & $14.3^{*}$ \\
\hline Write on board (upper part) & 7 & 12.6 & -9.8 & -10.2 & 1.8 & 2.3 \\
\hline Clean sink & 49.1 & $64.5^{*}$ & 18.5 & 18.8 & 7.4 & $12.6^{*}$ \\
\hline Pour water in a glass and drink & k 16.5 & 18.2 & 13.4 & 11.6 & 12.5 & 12.4 \\
\hline Stretch arms high and slowly & 35.9 & $44.9 *$ & -1.1 & -2.8 & 2.1 & 3.4 \\
\hline Draw a picture & 51.2 & $63.6^{*}$ & 13.2 & 13.6 & 5.4 & 6.5 \\
\hline Shake hands & 6.5 & 9.2 & 13.9 & 14.4 & 1.6 & 1.1 \\
\hline Fold up towels & 37.5 & 39.5 & 10.7 & 11.1 & 4.1 & 5 \\
\hline Put towels on the top shelf & 70.4 & $86.9 *$ & 3.9 & 6 & 7.9 & 9.9 \\
\hline Walk at preferred speed & 24.2 & $33.8^{*}$ & 19.1 & 19.2 & 40.6 & $51.9 *$ \\
\hline $\begin{array}{l}\text { Comb hair, brush teeth, wash } \\
\text { face }\end{array}$ & 38.5 & $46.9 *$ & 7.5 & 6.5 & 3.7 & 3.2 \\
\hline Sweep floor & 61.3 & 79.3 & 16.1 & 16.2 & 21.6 & 24.8 \\
\hline Rest & 5.5 & $8.8^{*}$ & 17.5 & 17.8 & 2.2 & 3.8 \\
\hline
\end{tabular}

*: sig. difference between COPD and healthy $(\mathrm{p}<0.05)$.

Abbreviations; a.u.: arbitrary unit.

Note: arm elevation is calculated using the mean low pass signal in the longitudinal direction (MI). Lower MI means higher arm elevation. 
Table 6.5: geometric means of relative muscle effort (\%). Differences between COPD and healthy were tested using the log10 transformed relative muscle effort.

\begin{tabular}{|c|c|c|c|c|c|c|}
\hline & \multicolumn{2}{|c|}{$\begin{array}{l}\text { Effort (\%) } \\
\text { Trapezius }\end{array}$} & \multicolumn{2}{|c|}{$\begin{array}{c}\text { Effort (\%) } \\
\text { Biceps }\end{array}$} & \multicolumn{2}{|c|}{$\begin{array}{c}\text { Effort (\%) } \\
\text { Deltiod }\end{array}$} \\
\hline & COPD & Healthy & COPD & Healthy & COPD & Healthy \\
\hline Clean window (upper part) & 52.1 & 38.6 & 26.1 & 24.1 & 35.7 & 41 \\
\hline Write on board (upper part) & 41.7 & $23.0^{*}$ & 14.4 & 12.2 & 21 & 19.5 \\
\hline Clean sink & 18.2 & 11.4 & 10.8 & 9.2 & 15.4 & 18.2 \\
\hline $\begin{array}{l}\text { Pour water in a glass and } \\
\text { drink }\end{array}$ & 23.1 & 15.4 & 8.1 & 5.6 & 5.1 & 6.7 \\
\hline Stretch arms high and slowly & 33.8 & 23.9 & 11.4 & 12 & 15.8 & 21.6 \\
\hline Draw a picture & 23 & 12.5 & 15.3 & 11.9 & 9.2 & 10.6 \\
\hline Shake hands & 13.4 & $8.1^{*}$ & 4.6 & 4.4 & 4.9 & 5.9 \\
\hline Fold up towels & 33.5 & 14.8 & 14.9 & 9.4 & 13.8 & 13.7 \\
\hline Put towels on the top shelf & 45.3 & $21.5^{*}$ & 20.3 & $13.6^{*}$ & 21.2 & 19.7 \\
\hline Walk at preferred speed & 11.4 & $4.8^{*}$ & 4.4 & 2.6 & 4.4 & 3.5 \\
\hline $\begin{array}{l}\text { Comb hair, brush teeth, wash } \\
\text { face }\end{array}$ & 33.3 & 26 & 19 & 17.5 & 9.7 & 13.4 \\
\hline Sweep floor & 21.2 & $11.8^{*}$ & 14.5 & 13 & 11.1 & 11.8 \\
\hline Rest & 7.7 & 4.7 & 3.1 & 2.2 & 2.7 & 2.3 \\
\hline
\end{tabular}

*: sig. difference between COPD and healthy $(\mathrm{p}<0.05)$. 

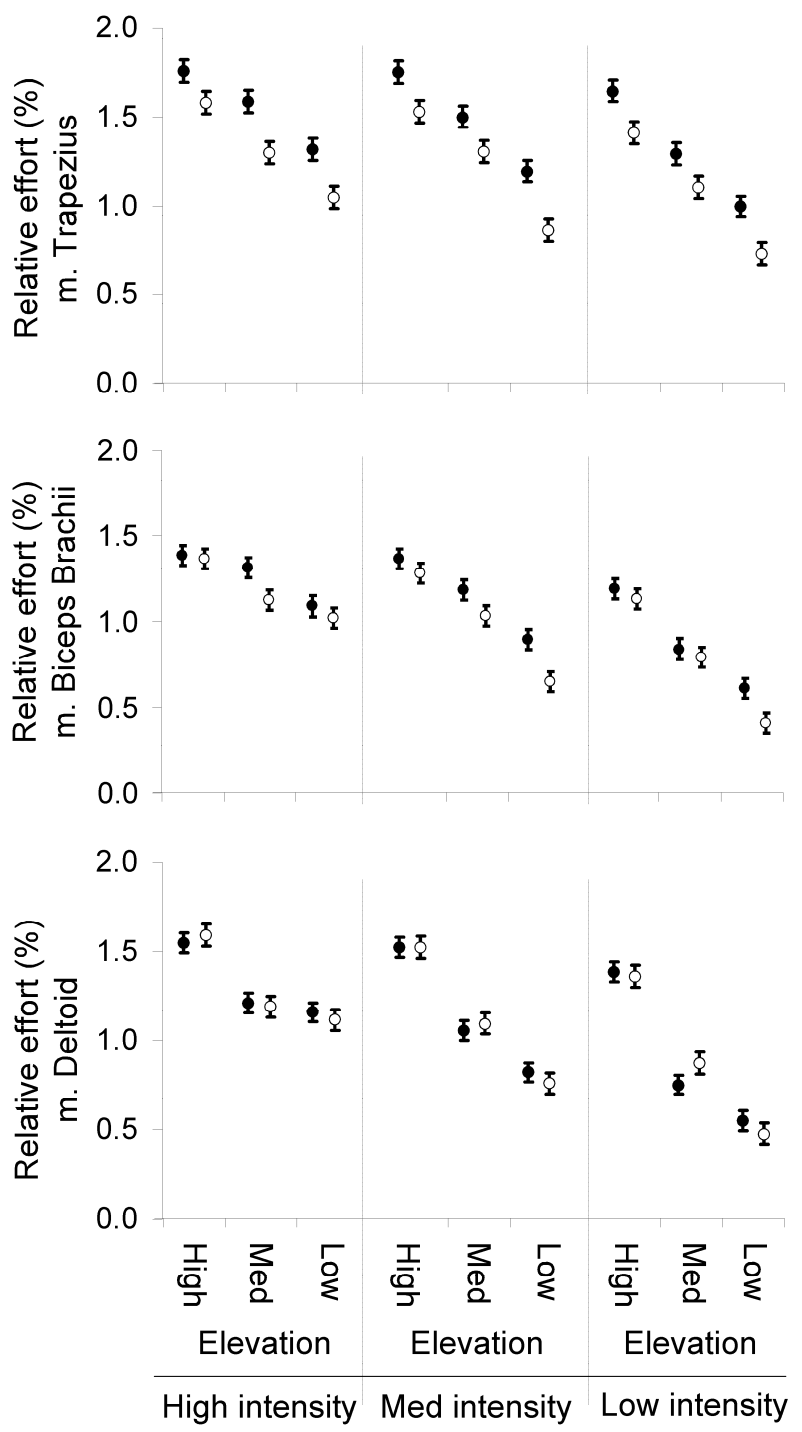

Figure 6.10: Log transformed relative muscle effort (\%) of the trapezius, biceps and deltoid muscles at different levels of arm elevation (high, medium low) and arm intensity (high, medium low) for COPD patients (closed rounds) and healthy subjects (open rounds). Error bars represent standardized errors of the mean (SEM).

Note: an average relative muscle effort difference on the $\log (10)$ scale of 0.332 between COPD and Healthy groups (e.g. for trapezius low elevation/medium intensity) means that on the original scale the relative muscle effort of COPD patients is increased ca. twofold compared to the healthy group (specifically multiplied by $100.332=2.147$, see also table 6.7 ). 
Table 6.6: Arm intensity (a.u.), arm elevation (a.u.) and leg intensity (a.u) during different arm categories for COPD patients and healthy subjects.

\begin{tabular}{|c|c|c|c|c|c|}
\hline Intensity & Elevation & Group & $\begin{array}{l}\text { Arm intensity } \\
\text { (a.u.) }\end{array}$ & $\begin{array}{l}\text { Arm elevation } \\
\text { (a.u.) }\end{array}$ & Leg intensity (a.u.) \\
\hline \multirow{6}{*}{ High } & \multirow{2}{*}{ High } & COPD & 68.1 & -7.5 & 7.5 \\
\hline & & Healthy & $81.3^{*}$ & -9.1 & $12.1^{*}$ \\
\hline & \multirow{2}{*}{ Medium } & COPD & 75.6 & 8.1 & 8.1 \\
\hline & & Healthy & 82.5 & 8.8 & 9.2 \\
\hline & \multirow{2}{*}{ Low } & COPD & 67.1 & 17.5 & 14.9 \\
\hline & & Healthy & 74.5 & 17.8 & 19.5 \\
\hline \multirow{6}{*}{ Medium } & \multirow{2}{*}{ High } & COPD & 35.7 & -10.3 & 3.8 \\
\hline & & Healthy & 35.8 & -11 & 3.6 \\
\hline & \multirow{2}{*}{ Medium } & COPD & 34.8 & 10.2 & 4.5 \\
\hline & & Healthy & 35.4 & 9.3 & 5.4 \\
\hline & \multirow{2}{*}{ Low } & COPD & 33.1 & 18.3 & 19.4 \\
\hline & & Healthy & 34.2 & 18.6 & $31.3^{*}$ \\
\hline \multirow{6}{*}{ Low } & \multirow{2}{*}{ High } & COPD & 8 & -10.3 & 1.7 \\
\hline & & Healthy & 9.8 & -11 & 1.7 \\
\hline & \multirow{2}{*}{ Medium } & COPD & 8.3 & 11.5 & 2.6 \\
\hline & & Healthy & 9.4 & 10.4 & 2.7 \\
\hline & \multirow{2}{*}{ Low } & COPD & 8.2 & 17.7 & 6.9 \\
\hline & & Healthy & 8.2 & 17.8 & 4.6 \\
\hline
\end{tabular}

*: sig. difference between COPD and healthy $(\mathrm{p}<0.05)$.

Abbreviations; a.u.: arbitrary unit.

Note: arm elevation is calculated using the mean low pass signal in the longitudinal direction (MI). Lower $\mathrm{MI}$ means higher arm elevation. 
Table 6.7: Geometric means of relative muscle effort (\%).

\begin{tabular}{|c|c|c|c|c|c|}
\hline Intensity & Elevation & Group & $\begin{array}{c}\text { Trapezius effort } \\
\text { (\%) }\end{array}$ & $\begin{array}{c}\text { Biceps } \\
\text { effort (\%) }\end{array}$ & $\begin{array}{c}\text { Deltoid } \\
\text { effort (\%) }\end{array}$ \\
\hline \multirow{6}{*}{ High } & \multirow{2}{*}{ High } & COPD & 56.7 & 24.1 & 35.4 \\
\hline & & Healthy & 37.8 & 23 & 39.3 \\
\hline & \multirow{2}{*}{ Medium } & COPD & 38.5 & 20.4 & 16.2 \\
\hline & & Healthy & 19.9 & 13.3 & 15.5 \\
\hline & \multirow{2}{*}{ Low } & COPD & 20.7 & 12.2 & 14.4 \\
\hline & & Healthy & 11.1 & 10.5 & 13.1 \\
\hline \multirow{6}{*}{ Medium } & \multirow{2}{*}{ High } & COPD & 56.3 & 23 & 33.6 \\
\hline & & Healthy & 33.8 & 19 & 33.6 \\
\hline & \multirow{2}{*}{ Medium } & COPD & 31.7 & 15.3 & 11.4 \\
\hline & & Healthy & 20.2 & 10.8 & 12.5 \\
\hline & \multirow{2}{*}{ Low } & COPD & 15.6 & 7.9 & 6.6 \\
\hline & & Healthy & 7.3 & 4.4 & 5.8 \\
\hline \multirow{6}{*}{ Low } & \multirow{2}{*}{ High } & COPD & 44.2 & 15.5 & 24.3 \\
\hline & & Healthy & 25.8 & 13.5 & 23.1 \\
\hline & \multirow{2}{*}{ Medium } & COPD & 19.6 & 6.9 & 5.7 \\
\hline & & Healthy & 12.8 & 6.2 & 7.5 \\
\hline & \multirow{2}{*}{ Low } & COPD & 9.9 & 4.1 & 3.5 \\
\hline & & Healthy & 5.4 & 2.6 & 3 \\
\hline
\end{tabular}

Note: A random intercept model analysis was conducted to test significance of the effects. Three-way interactions were present for all muscles. 
Table 6.8: Time spent in different arm categories during arm ADL protocol

\begin{tabular}{|c|c|c|c|c|c|}
\hline \multicolumn{2}{|c|}{ Arm category } & \multicolumn{2}{|c|}{ Time (sec) } & \multicolumn{2}{|c|}{ Statistics } \\
\hline Intensity & Elevation & COPD & Healthy & $95 \% \mathrm{Cl}$ & $P$ value \\
\hline \multirow{3}{*}{ High } & High & $18 \pm 8$ & $24 \pm 6$ & $-11--1$ & 0.03 \\
\hline & Medium & $31 \pm 15$ & $42 \pm 12$ & $-20-0$ & 0.042 \\
\hline & Low & $21 \pm 10$ & $36 \pm 13$ & $-23--7$ & 0.001 \\
\hline \multirow{3}{*}{ Medium } & High & $18 \pm 8$ & $15 \pm 9$ & $-2-9$ & 0.221 \\
\hline & Medium & $33 \pm 14$ & $28 \pm 13$ & $-5-14$ & 0.334 \\
\hline & Low & $50 \pm 20$ & $44 \pm 16$ & $-6-19$ & 0.317 \\
\hline \multirow{3}{*}{ Low } & High & $24 \pm 4$ & $19 \pm 9$ & $-1-8$ & 0.11 \\
\hline & Medium & $27 \pm 9$ & $23 \pm 13$ & $-4-12$ & 0.282 \\
\hline & Low & $39 \pm 14$ & $30 \pm 11$ & $-1-17$ & 0.091 \\
\hline
\end{tabular}

Abbreviations; $\mathrm{Cl}$ : Confidence Interval of the mean difference.

Within the typical arm category for walking (medium intensity in combination with a low arm elevation) arm muscle efforts of all muscles were significantly lower with the presence of leg activity (leg muscles initiated the movement and not the arm muscles) compared to without the presence of leg activity ( $p<0.05$, figure 6.11).

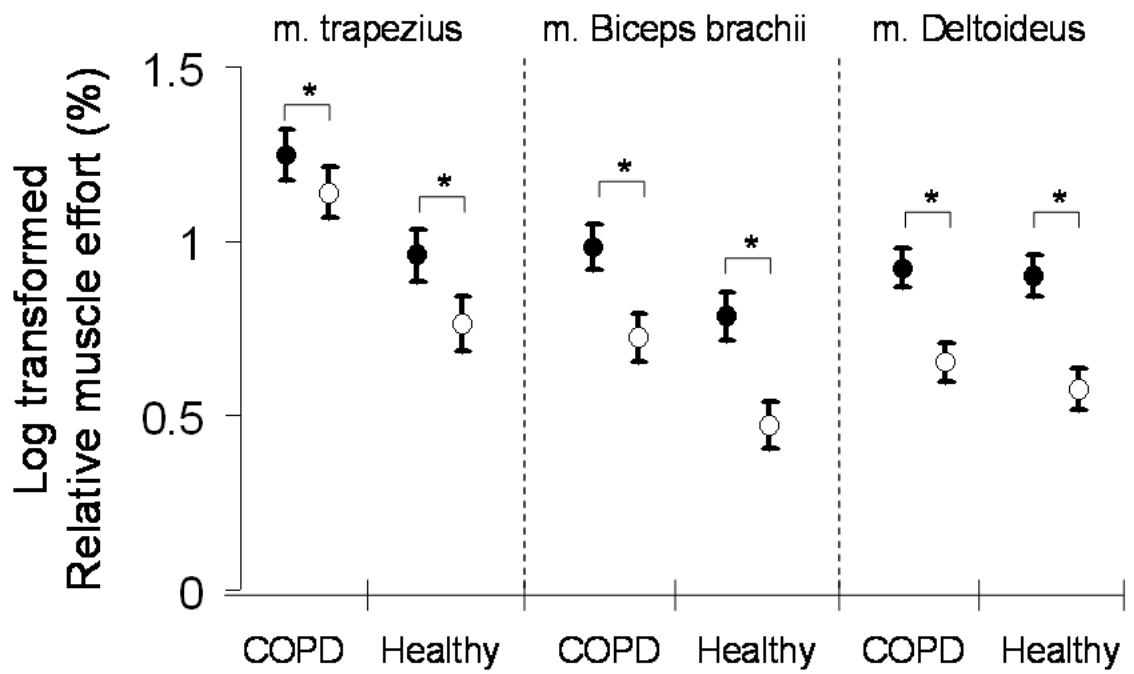

Figure 6.11: $\log (10)$ transformed relative muscle effort (\%) of the trapezius, biceps and deltoid muscles for the arm category 'low elevation and medium intensity' (typically the arm category during walking). Data is presented for COPD patients and healthy subjects during leg activity (white rounds) and without leg activity (e.g. sitting and standing) (black rounds). 


\section{Discussion}

This study is the first characterizing daily arm activities and associated relative effort of upper body muscles in patients with mild to severe COPD. COPD patients spent more time in arm postures versus arm activities. Furthermore, the arm movement intensity was lower compared to healthy elderly subjects. After correction for walking, the time spent on daily arm activities was similar between patients with COPD and healthy subjects, while the intensity of arm movements remained significantly lower in patients with COPD. Also in a laboratory setting, the majority of the arm ADLs were performed with lower movement intensity by COPD patients. Despite that, COPD patients had a higher task-related trapezius muscle effort during several domestic ADLs compared to healthy elderly subjects.

Many COPD patients entering pulmonary rehabilitation experience arm activities to be problematic [5]. This study shows that in daily life COPD patients spent the same amount of time on arm activities during sitting and standing as healthy peers, although arm activities are poorly tolerated by COPD patients [9]. Arm activity time may be preserved because many arm activities are necessary for independent living (i.e., preparing food, self-care) $[7,8]$.

Though COPD patients spent as much time on arm activities during sitting and standing compared to healthy subjects, other characteristics of daily arm activities differed compared to the control group. Arm ADLs were performed at a significant lower intensity. These results implicate that COPD patients perform less daily tasks involving the arms or at a lower frequency [30].

Measurements in a laboratory setting allowed for a more detailed analysis regarding performance of daily activities in relation to maximal muscle effort. Similar to the daily life situation, COPD patients performed the instructed activities generally with lower arm movement intensity. However, the relative effort compared to maximum of the shoulder/arm muscles was similar or higher compared to healthy elderly subjects (table E1-E2). Increased relative muscle effort in COPD patients during specific ADLs was found for the trapezius muscle, while muscle effort for the biceps and deltoid muscles were similar to those of healthy elderly. These results suggest that COPD patients perform daily arm activities at a similar relative biceps and deltoid muscle effort as healthy subjects by compensating the intensity of their arm movements.

Relative muscle effort was compared between groups, arm elevation levels and movement intensity in a more detailed study. In COPD patients the trapezius muscle was clearly used at a higher degree of relative muscle effort, while relative muscle 
effort for the other muscles was group independent. First, this is likely explained by the inspiratory function of the trapezius muscle [17] and breathing is demanding for COPD patients. Second, it is in line with the finding that during walking, which increases ventilator requirements [31], relative effort of the trapezius muscle of COPD patients was much higher compared to healthy subjects. Furthermore differences in trapezius muscle effort between groups increased with arm elevation. This can be explained by previous findings showing that arm elevation in COPD patients is associated with changes in lung volumes and loss of vital capacity $[9,10,11,12,13,14,15,16]$.

Methodological considerations are described in the online supplement. Whether and to what extent pulmonary rehabilitation can change daily arm activities in terms of duration per day, its levels and movement intensity, remains currently unknown. Moreover, a reduction of the task-related burden needs to be considered by learning and applying energy conservation techniques [32]. These techniques previously resulted in a significant decrease in task-related oxygen uptake during the performance of domestic ADLs in COPD [30]. Future studies should evaluate the effects of different tailored interventions on arm ADLs in patients with COPD.

Concluding, patients with COPD have similar duration of daily arm activities compared to healthy elderly subjects after correction for walking, but perform arm activities at a lower intensity. Moreover, patients perform some daily arm activities at a higher proportion of their maximum trapezius muscle effort. Future studies are warranted to assess the effects of a tailored pulmonary rehabilitation program on arm ADLs in patients with COPD.

\section{Acknowledgments}

The authors are grateful to the volunteers who participated in the present study. Moreover Yvonne van Dooren, Nika Ritsema and Paul Willems of Human Movement Science, NUTRIM School for Nutrition, Toxicology and Metabolism are gratefully acknowledged for their help with data collection and electronic data processing. 


\section{References}

1. Katz P, Chen H, Omachi TA, Gregorich SE, Julian L, et al. (2011) The Role of Physical Inactivity in Increasing Disability Among Older Adults With Obstructive Airway Disease. J Cardiopulm Rehabil Prev 31: 193-197.

2. Waschki B, Kirsten A, Holz O, Muller KC, Meyer T, et al. (2011) Physical Activity Is the Strongest Predictor of All-Cause Mortality in Patients With COPD: A Prospective Cohort Study. Chest 140: 331 342.

3. Garcia-Aymerich J, Lange P, Benet M, Schnohr P, Anto JM (2006) Regular physical activity reduces hospital admission and mortality in chronic obstructive pulmonary disease: a population based cohort study. Thorax 61: 772-778.

4. Annegarn J, Spruit MA, Savelberg HH, Willems PJ, van Bool C, et al. (2012) Differences in Walking Pattern during 6-Min Walk Test between Patients with COPD and Healthy Subjects. PLoS One 7: e37329.

5. Annegarn J, Meijer K, Passos VL, Stute K, Wiechert J, et al. (2012) Problematic activities of daily life are weakly associated with clinical characteristics in COPD. J Am Med Dir Assoc 13: 284-290.

6. Vaes AW, Wouters EF, Franssen FM, Uszko-Lencer NH, Stakenborg KH, et al. (2011) Task-related oxygen uptake during domestic activities of daily life in patients with COPD and healthy elderly subjects. Chest 140: 970-979.

7. Guccione AA, Felson DT, Anderson JJ, Anthony JM, Zhang Y, et al. (1994) The effects of specific medical conditions on the functional limitations of elders in the Framingham Study. Am J Public Health 84: 351-358.

8. Rodriguez Gonzalez-Moro JM, de Lucas Ramos P, Izquierdo Alonso JL, Lopez-Muniz Ballesteros B, Anton Diaz E, et al. (2009) Impact of COPD severity on physical disability and daily living activities: EDIP-EPOC I and EDIP-EPOC II studies. Int J Clin Pract 63: 742-750.

9. Dolmage TE, Maestro L, Avendano MA, Goldstein RS (1993) The ventilatory response to arm elevation of patients with chronic obstructive pulmonary disease. Chest 104: 1097-1100.

10. Celli BR, Rassulo J, Make BJ (1986) Dyssynchronous breathing during arm but not leg exercise in patients with chronic airflow obstruction. N Engl J Med 314: 1485-1490.

11. Lebzelter J, Klainman E, Yarmolovsky A, Sulkes J, Fink-Krelbaum T, et al. (2001) Relationship between pulmonary function and unsupported arm exercise in patients with COPD. Monaldi Arch Chest Dis 56: 309-314.

12. Martinez FJ, Couser JI, Celli BR (1991) Respiratory response to arm elevation in patients with chronic airflow obstruction. Am Rev Respir Dis 143: 476-480.

13. Baarends EM, Schols AM, Slebos DJ, Mostert R, Janssen PP, et al. (1995) Metabolic and ventilatory response pattern to arm elevation in patients with COPD and healthy age-matched subjects. Eur Respir J 8: 1345-1351.

14. Criner GJ, Celli BR (1988) Effect of unsupported arm exercise on ventilatory muscle recruitment in patients with severe chronic airflow obstruction. Am Rev Respir Dis 138: 856-861.

15. Gigliotti F, Coli C, Bianchi R, Grazzini M, Stendardi L, et al. (2005) Arm exercise and hyperinflation in patients with COPD: effect of arm training. Chest 128: 1225-1232.

16. Epstein SK, Celli BR, Williams J, Tarpy S, Roa J, et al. (1995) Ventilatory response to arm elevation. Its determinants and use in patients with chronic obstructive pulmonary disease. Am J Respir Crit Care Med 152: 211-216.

17. (2002) ATS/ERS Statement on respiratory muscle testing. Am J Respir Crit Care Med 166: 518-624.

18. Franssen FM, Broekhuizen R, Janssen PP, Wouters EF, Schols AM (2005) Limb muscle dysfunction in COPD: effects of muscle wasting and exercise training. Med Sci Sports Exerc 37: 2-9. 
19. Franssen FM, Wouters EF, Baarends EM, Akkermans MA, Schols AM (2002) Arm mechanical efficiency and arm exercise capacity are relatively preserved in chronic obstructive pulmonary disease. Med Sci Sports Exerc 34: 1570-1576.

20. Clark CJ, Cochrane LM, Mackay E, Paton B (2000) Skeletal muscle strength and endurance in patients with mild COPD and the effects of weight training. Eur Respir J 15: 92-97.

21. Gea JG, Pasto M, Carmona MA, Orozco-Levi M, Palomeque J, et al. (2001) Metabolic characteristics of the deltoid muscle in patients with chronic obstructive pulmonary disease. Eur Respir J 17: 939945.

22. Annegarn J, Spruit MA, Schols AM, Savelberg HH, murgia A, et al. Assessment of intensity and elevation of arm movements during activities of daily life. Submitted.

23. Spruit MA, Vanderhoven-Augustin I, Janssen PP, Wouters EF (2008) Integration of pulmonary rehabilitation in COPD. Lancet 371: 12-13.

24. Annegarn J, Spruit MA, Uszko-Lencer NH, Vanbelle S, Savelberg HH, et al. (2011) Objective physical activity assessment in patients with chronic organ failure: a validation study of a new single-unit activity monitor. Arch Phys Med Rehabil 92: 1852-1857 e1851.

25. Quanjer PH, Tammeling GJ, Cotes JE, Pedersen OF, Peslin R, et al. (1993) Lung volumes and forced ventilatory flows. Report Working Party Standardization of Lung Function Tests, European Community for Steel and Coal. Official Statement of the European Respiratory Society. Eur Respir J Suppl 16: 5-40.

26. Allaire J, Maltais F, Doyon JF, Noel M, LeBlanc $P$, et al. (2004) Peripheral muscle endurance and the oxidative profile of the quadriceps in patients with COPD. Thorax 59: 673-678.

27. Theou O, Jones GR, Vandervoort AA, Jakobi JM (2010) Daily muscle activity and quiescence in nonfrail, pre-frail, and frail older women. Exp Gerontol 45: 909-917.

28. Pitta F, Troosters T, Spruit MA, Probst VS, Decramer M, et al. (2005) Characteristics of physical activities in daily life in chronic obstructive pulmonary disease. Am J Respir Crit Care Med 171: $972-$ 977.

29. Rabe KF, Hurd S, Anzueto A, Barnes PJ, Buist SA, et al. (2007) Global strategy for the diagnosis, management, and prevention of chronic obstructive pulmonary disease: GOLD executive summary. Am J Respir Crit Care Med 176: 532-555.

30. Velloso M, Jardim JR (2006) Study of energy expenditure during activities of daily living using and not using body position recommended by energy conservation techniques in patients with COPD. Chest 130: $126-132$.

31. Marquis N, Debigare R, Bouyer L, Saey D, Laviolette L, et al. (2009) Physiology of Walking in Patients with Moderate to Severe Chronic Obstructive Pulmonary Disease. Med Sci Sports Exerc 41: 15401548.

32. Velloso M, Jardim JR (2006) Functionality of patients with chronic obstructive pulmonary disease: energy conservation techniques. J Bras Pneumol 32: 580-586. 
Chapter 7

General discussion 


\section{General discussion}

Many COPD patients experience problems with activities of daily life (ADLs) that cause loss of independence and social isolation and thus greatly reduce their quality of life $[1,2,3]$. Regular physical activity is extremely important to preserve functional status $[5,6]$. Because functional status is the most important factor affecting health related quality of life and health care utilization in chronic disease and old age [7], insight in problematic ADLs and valid outcome measures of daily physical activity are of key importance to evaluate the success of treatment programs for COPD patients. This thesis has therefore addressed the problematic ADLS COPD patients experience and focused on the assessment of valid diagnostic and outcome measures of daily physical activity of COPD patients entering pulmonary rehabilitation.

\section{Problematic activities in daily life; a patient's perspective}

The most prevalent problematic ADLs of COPD patients were identified using the Canadian Occupational Performance Measure (COPM) in chapter 2. In this study 820 COPD patients entering pulmonary rehabilitation reported 2999 problematic ADLs. Walking was found to be the most prevalent problematic ADL. In addition this study determined the perceived performance and satisfaction scores for the identified problematic ADLs. Known clinical determinants of ADLs in COPD patients, like age, FEV1, functional exercise performance and psychological status, were only weakly associated with performance and satisfaction scores of problematic ADLs. These findings indicate that interview based performance measures are needed for identification of ADLs that an individual perceives as problematic to perform, to allow tailored intervention.

The performance and satisfaction scores of the COPM reflect the functional status of the individual patients. Besides the COPM many other measures exist that evaluate the functional performance. Recently the COPM was well graded among 25 other instruments for the assessment of functional performance in a review of Kocks et al. [8]. The Medical Research Council (MRC) dyspnea questionnaire and the functional status domain of the Clinical COPD Questionnaire (CCQ) were found to be the best patient-reported outcome tools to assess functional performance in COPD patients [8]. However, the MRC and CCQ, as well as the other instruments listed within the review by Kocks et al. assess only information on pre-defined daily activities [8]. The results of chapter 2, showing the diversity of problematic activities reported by COPD patients, indicate that these pre-defined activities may not al- 
ways be most important to the individual patient and thereby poorly indentify patients' individual needs [9]. For this reason the COPM is a unique instrument that enables an exploration of the individual problematic ADLs $[2,10]$.

COPD patients have an enormous variety of problematic ADLs which involve both the lower body (e.g. walking, cycling) and the upper body (e.g. doing laundry, gardening) (Chapter 2). These activities are of great importance, as they limit patients to become more active in daily life. Each problematic activity can have different underlying factors responsible for the limitations and may therefore postulate different treatment approaches. It is therefore important to study frequent problematic activities in more detail to obtain insight in the underlying factors responsible for the limitations during the performance of these activities. While the most prevalent problematic ADL of COPD patients is walking, no study has yet investigated walking of COPD patients in detail, other than the one that we conducted (chapter 4).

\section{Technological developments}

Specific hardware and software was needed to enable data gathering and data analysis of daily physical activity of lower and upper extremities. Commercial available accelerometer-based activity monitors usually have a fixed sensor configuration, sample frequency, battery life, and memory capacity. Moreover raw data is frequently not stored. Instead a set of pre-defined outcomes are available as well as specific instruction on the location of the sensor(s) on the body. Furthermore, no information is available on the algorithms used to transform raw data into the predefined outcomes. Performance requirements of accelerometer-based activity monitors vary for different applications. At the start of our study no commercial available activity monitor met our requirements for studying upper and lower limb performance in daily life. Therefore a new activity monitor (CAM) was developed by Maastricht Instruments. The validity of the CAM to assess lower and upper extremity activity in daily life has been described in this thesis (chapter 3 and chapter 5). Since the start of these validation studies activity monitoring is increasingly used in studies involving COPD patients to analyze performance of daily activities $[49,50,51,52,53,54,55,56,57,58]$. Still, the majority of studies involving COPD patients obtain overall measures of physical activity such as activity counts [59], movement intensity [59], steps per day $[60,61,62]$, time spent in activities above an estimated energy expenditure [61,62] and duration of different activity types (walking, standing, sitting and lying down) $[55,63,64]$. The possibility to work from raw accelerometer signals in this thesis has resulted to more detailed information on the performance of specific activities important to the COPD patients. 
The studies of this thesis fit well in the current trend that activity monitoring methods rapidly develop and new possibilities arise for data gathering and data analysis [65]. Important considerations for data gathering are the choice of sensors, the amount of sensors, sensor placements, the activities analyzed and the populations of which activities are studied. For data analysis it is important to have a fundamental understanding of how specific movements and postures produce characteristic body-worn sensor signal. This understanding helps to select relevant features that describe movements. Because a specific study aim requires specific data gathering and data analysis, there is current limited standardization in methodology. A positive side effect of this pluriformity is that it creates the opportunity for cross learning. For example the method described in this thesis for evaluating arm activity in COPD patients (chapters 5 and 6) was very much inspired by methods that were previously developed to evaluate patients with stroke or shoulder pathology $[66,67]$. The methods described in this thesis may also be relevant for chronic heart failure patients who also experience problems with daily activities that cause loss of independence and social isolation and thus greatly reduce their quality of life $[1,3]$.

\section{Objective activity assessment}

Walking is the most frequently reported problematic activity in daily life by COPD patients (chapter 2). Many factors have shown to influence the walking pattern. Examples of these factors are being older [13], having a chronic disease (e.g. diabetes neuropathy [14], stoke [15] and impaired cognition [16]), being fatigued in the leg muscles [12], being more prone to fall $[17,18,19]$, walking without arm swing [20], walking without vision [21] and walking under different circumstances (e.g. surface [22] and walking speed [23]). Based on these previous findings it is not surprising that COPD patients who are on average 65 years old [24], are prone to fall [25], walk with reduces arm swing [26], rapidly fatigue leg muscles [27] and have many co-morbidities [28] show an altered walking pattern. In chapter 4 of this thesis it was shown that COPD patients walk on average with a lower intensity, a lower cadence and show a higher variability of the accelerometer signal during 6-min walk test in comparison with healthy elderly subjects. The difference in this variability remained even if patients and healthy controls with the same walking speed were compared.

The underlying mechanisms that are responsible for the altered walking pattern reported in chapter 4 could not be elucidated from the study and may differ for the individual patient with COPD. Nevertheless, our findings generate a clear rationale to study walking patterns in more detail. More specifically, walking pattern should be further studied under different circumstances and including measures reflecting 
underlying mechanisms responsible for the altered walking patterns. Motion capture systems [29], force plates [30] and electromyography (EMG) [31,32] are well suited to determine the neuromuscular-skeletal contributions to a specific walking pattern. As such, it provides quantitative information to help prescribe treatment and assess its outcome. Previously it was shown that specific training can improve walking in diabetic patients $[33,34,35]$ and reduce both the rate of falls and the risk of falling in at-risk elderly community-dwelling adults [36]. Inertial sensors, like accelerometers, gyroscopes, magnetometers and pressure sensors can evaluate the walking patterns under different circumstances outside the laboratory in the patients own environment [37]. Such studies can be essential for new insights in treatments strategies to increase performance and satisfaction with walking in daily life as assessed in chapter 2 . This may be of key importance to facilitate a more active lifestyle.

Arm activities are required for maintenance of self-care and independent living. Some of these activities, like dressing and undressing, are within the top ten most frequently reported problematic activities for COPD patients (chapter 2). It is recognized that activities of daily life involving arms, especially at higher arm elevation levels without support, are poorly tolerated by patients with COPD [38]. Nevertheless, we found in chapter 6 that during sedentary activities (e.g. sitting and standing) in daily life COPD patients spent the same amount of time on arm activities as healthy peers. In the same group of patients the arm muscle function was affected to a smaller degree compared to the leg muscle function, which is in line with previous studies $[39,40,41,42]$. While the oxidative capacity of the vastus lateralis muscle of patients with moderate-to-severe COPD was significantly reduced $[43,44]$, the oxidative capacity in the musculature of the upper extremity was not affected [40]. Also, the mechanical efficiency was lower in leg muscles, while arm mechanical efficiency was not significantly affected [42]. Based on these findings it seems that COPD patients have the tendency to eliminate first those activities that involve the muscles of ambulation, leading to overall deconditioning. Arm activities are less effected as they are still required for maintenance of self-care and independent living (i.e., preparing food, cleaning, self-care) $[5,6]$, even if they induce uncomfortable symptoms of dyspnea and fatigue. As a consequence COPD patients may avoid leaving their homes to limit the burden and get socially disconnected.

Arm activity duration in daily life is preserved in COPD patients (chapter 6). Nevertheless, improvements in upper-limb strength or endurance resulting from training could lead to improved overall functional capacity and ability to perform daily life activities. For example, unsupported arm exercise training improved peak arm exercise capacity, metabolic and ventilatory demands and symptoms such as perceived arm exertion and dyspnea [45]. For this reason arm training during rehabilitation, as 
recommended by the joint American College of Chest Physicians and the American Association of Cardiovascular and Pulmonary Rehabilitation guidelines [46], has an important function. Moreover a reduction of the task-related burden can to be considered by learning and applying energy conservation techniques as part of a comprehensive pulmonary rehabilitation program [47]. Velloso and Jardim showed a significant decrease in task-related oxygen uptake and dyspnea while using energy conservation techniques during the performance of domestic activities in daily life in COPD [48]. Future studies should evaluate the effects of different tailored interventions on arm ADLs in patients with COPD. Arm activity monitors can help to objectify the arm performance during daily life activities.

\section{Methodological considerations}

The present thesis has some important limitations that should be considered in interpreting the results. First, all patients included in the studies entered the assessment for pulmonary rehabilitation at $\mathrm{CIRO}+$, Horn. This may have limited the generalizability of the results. However, patient characteristics described in this thesis were very similar to those reported in other studies. For example, time spent in different postures and activity reported in chapter 6 were very similar to values reported by Pitta and colleagues [70]. Second, differences in walking patterns between COPD and healthy subjects were investigated in chapter 4 during a specific test where people had to walk as fast as possible within 6 minutes. Although these walking patterns clearly explain differences in walking distance, these walking patterns may not be representative for daily function. Therefore it is important to repeat the measurements during daily routine to show the relevance of the measures outside the clinical setting. Third, in chapter 6 we measured daily activities both in the home environment as well as in a standardized setting. In the home environment activity monitors were programmed to start at 7am and finish at $7 \mathrm{pm}$ for energy conservation purposes. As a result important activities performed in the evenings, like cooking, showering and brushing teeth were perhaps not measured. Moreover, the activity monitors were not water proof. As a result subjects had to take the devices of while showering or swimming. Showering is the fourth most prevalent problematic activity for COPD patients (chapter 2) and it would therefore have been interesting to include it in the data collection. However, to be able to compare the performance of a specific activity like showering between COPD patients and healthy subjects, the daily activity needs to be recognized from the accelerometer signals. This requires additional studies to explore novel data mining techniques for improving physical activity recognition. Fourth, in this thesis only cross-sectional data was collected due to time limitations. Therefore we could not take seasonal influences on measures of daily activity into account or study the 
effect of a pulmonary rehabilitation program. Last, performances in the patients own environments are most likely not the same as in a clinical setting. Therefore the performances of activities of interest are preferably studied in the home environment. Unfortunately it is not always possible to retrieve which recorded data in daily life belongs to the activity of interest due to the variety and complexity of daily movement, especially of movements involving the arms (chapter 5). Moreover, in the home environment it is not feasible to measure muscle function simultaneously for long time periods in an unobtrusive way. Therefore measuring in a standardized setting was unavoidable.

\section{Recommendations for future research}

COPD patients have an enormous variety of problematic ADLs. Moreover, COPD patients perform walking and daily arm activities differently compared to healthy subjects. Possible underlying mechanisms that may cause altered performance have been discussed in this thesis. However, future studies are warranted to support the present findings and to elucidate which underlying mechanisms contribute to the altered performance of daily activities in COPD patients.

Activity monitors have promising potential to become part of telecare for elderly people suffering from chronic diseases [68]. Telecare aims to increase patient's independence and quality of life, and produce cost savings for the society. Activity monitors in combination with advanced ICT solutions (e.g. in smartphones and tablets) could monitor and assist elderly with chronic conditions in their home environment [69]. Based on such technology, interventions can be introduced in the home environment in an easier manner, and tailored to the individual needs.

New technology for activity monitoring should be greatly accepted by the users (elderly, caregivers), unobtrusive (e.g. easy to use, water proof, long lasting batteries, wireless) and cost effective. These important aspects require additional attention in future studies evaluating activity monitors in elderly subjects. Team working among multidisciplinary researchers, IT engineers, medical and social scientists is essential in order to build systems based on a better understanding of the people being monitored.

Walking variability is higher in the medio-lateral direction in COPD patients compared to the control group (this thesis), suggesting larger balance disturbances. This may also explain partially why patients with COPD experience abnormalities with day-to-day walking, including falls. Falls and related injuries are life threatening to older people and herald an inability to live independently. For this reason it is important that further studies evaluate the effect of walking and balance training on walking abnormalities and fall risk in COPD patients. 
Most sedentary older adults and individuals living with disability and chronic illness are more limited in their everyday activities. It is increasingly recognized that these individuals can still benefit from a physically active lifestyle. However, it is not yet clear if a general promotion of activity level for elderly with disability and chronic illness is appropriate. Conceivably an individualized and continuous assessment and treatment of their limitations contributing to their inactivity is needed first. Future research should elucidate the value of this approach compared to general activity promotion. Moreover activity monitors may serve well as coaching tools to maintain and/or enhance a more physical active lifestyle after an intervention.

The degree to which participation in tailored interventions translates into enhanced performance of everyday life activities is not yet clear. Therefore, the effect of tailored interventions on the performance of problematic activities in the home environment needs to be evaluated in future research. For example, arm training is considered as an important part of pulmonary rehabilitation, but the effect of these trainings on daily arm performance is unknown. Arm activity monitors can help to objectify the performance of daily life activities during these studies. 


\section{References}

1. Griffith L, Raina P, Wu H, Zhu B, Stathokostas L (2010) Population attributable risk for functional disability associated with chronic conditions in Canadian older adults. Age Ageing 39: 738-745.

2. Williams V, Bruton A, Ellis-Hill C, McPherson K (2007) What really matters to patients living with chronic obstructive pulmonary disease? An exploratory study. Chron Respir Dis 4: 77-85.

3. Jeon YH, Kraus SG, Jowsey T, Glasgow NJ (2010) The experience of living with chronic heart failure: a narrative review of qualitative studies. BMC Health Serv Res 10: 77.

4. Hartman JE, Boezen HM, de Greef MH, Bossenbroek L, ten Hacken NH (2011) Consequences of physical inactivity in chronic obstructive pulmonary disease. Expert Rev Respir Med 4: 735-745.

5. Guccione AA, Felson DT, Anderson JJ, Anthony JM, Zhang Y, et al. (1994) The effects of specific medical conditions on the functional limitations of elders in the Framingham Study. Am J Public Health 84: 351-358.

6. Rodriguez Gonzalez-Moro JM, de Lucas Ramos P, Izquierdo Alonso JL, Lopez-Muniz Ballesteros B, Anton Diaz E, et al. (2009) Impact of COPD severity on physical disability and daily living activities: EDIP-EPOC I and EDIP-EPOC II studies. Int J Clin Pract 63: 742-750.

7. Ferrucci L, Baldasseroni S, Bandinelli S, de Alfieri W, Cartei A, et al. (2000) Disease severity and health-related quality of life across different chronic conditions. J Am Geriatr Soc 48: 1490-1495.

8. Kocks JW, Asijee GM, Tsiligianni IG, Kerstjens HA, van der Molen T (2011) Functional status measurement in COPD: a review of available methods and their feasibility in primary care. Prim Care Respir J 20: 269-275.

9. Stull DE, Leidy NK, Jones PW, Stahl E (2007) Measuring functional performance in patients with COPD: a discussion of patient-reported outcome measures. Curr Med Res Opin 23: 2655-2665.

10. Leidy NK, Haase JE (1996) Functional performance in people with chronic obstructive pulmonary disease: a qualitative analysis. ANS Adv Nurs Sci 18: 77-89.

11. Donelan JM, Kram R, Kuo AD (2001) Mechanical and metabolic determinants of the preferred step width in human walking. Proc Biol Sci 268: 1985-1992.

12. Helbostad JL, Leirfall S, Moe-Nilssen R, Sletvold O (2007) Physical fatigue affects gait characteristics in older persons. J Gerontol A Biol Sci Med Sci 62: 1010-1015.

13. Menz HB, Lord SR, Fitzpatrick RC (2003) Age-related differences in walking stability. Age Ageing 32: 137-142.

14. Menz HB, Lord SR, St George R, Fitzpatrick RC (2004) Walking stability and sensorimotor function in older people with diabetic peripheral neuropathy. Arch Phys Med Rehabil 85: 245-252.

15. Mizuike C, Ohgi S, Morita S (2009) Analysis of stroke patient walking dynamics using a tri-axial accelerometer. Gait Posture 30: 60-64.

16. Lamoth CJ, van Deudekom FJ, van Campen JP, Appels BA, de Vries OJ, et al. (2011) Gait stability and variability measures show effects of impaired cognition and dual tasking in frail people. J Neuroeng Rehabil 8: 2.

17. Senden R, Savelberg H, Grimm B, Heyligers I, Meijer K (in press) Accelerometry-based gait analysis, an additional objective approach to screen subjects at risk for falling. Gait Posture.

18. Bautmans I, Jansen B, Van Keymolen B, Mets T (2011) Reliability and clinical correlates of 3Daccelerometry based gait analysis outcomes according to age and fall-risk. Gait Posture 33: 366-372.

19. Hamacher D, Singh NB, Van Dieen JH, Heller MO, Taylor WR Kinematic measures for assessing gait stability in elderly individuals: a systematic review. J R Soc Interface 8: 1682-1698.

20. Kuo AD, Donelan JM (2010) Dynamic principles of gait and their clinical implications. Phys Ther 90 : 157-174.

21. Helbostad JL, Vereijken B, Hesseberg K, Sletvold O (2009) Altered vision destabilizes gait in older persons. Gait Posture 30: 233-238. 
22. Menz HB, Lord SR, Fitzpatrick RC (2003) Acceleration patterns of the head and pelvis when walking on level and irregular surfaces. Gait Posture 18: 35-46.

23. Helbostad JL, Moe-Nilssen R (2003) The effect of gait speed on lateral balance control during walking in healthy elderly. Gait Posture 18: 27-36.

24. Lacasse $Y$, Brosseau L, Milne S, Martin S, Wong E, et al. (2002) Pulmonary rehabilitation for chronic obstructive pulmonary disease. Cochrane Database Syst Rev: CD003793.

25. Roig M, Eng JJ, Maclntyre DL, Road JD, FitzGerald JM, et al. (2011) Falls in people with chronic obstructive pulmonary disease: an observational cohort study. Respir Med 105: 461-469.

26. Ortega JD, Fehlman LA, Farley CT (2008) Effects of aging and arm swing on the metabolic cost of stability in human walking. J Biomech 41: 3303-3308.

27. Seymour JM, Spruit MA, Hopkinson NS, Natanek SA, Man WD, et al. The prevalence of quadriceps weakness in COPD and the relationship with disease severity. Eur Respir J 36: 81-88.

28. Vogelmeier CF, Wouters EF Treating the systemic effects of chronic obstructive pulmonary disease. Proc Am Thorac Soc 8: 376-379.

29. Simon SR (2004) Quantification of human motion: gait analysis-benefits and limitations to its application to clinical problems. J Biomech 37: 1869-1880.

30. Sutherland DH (2005) The evolution of clinical gait analysis part III--kinetics and energy assessment. Gait Posture 21: 447-461.

31. Frigo C, Crenna P (2009) Multichannel SEMG in clinical gait analysis: a review and state-of-the-art. Clin Biomech (Bristol, Avon) 24: 236-245.

32. Marquis N, Debigare R, Bouyer L, Saey D, Laviolette L, et al. (2009) Physiology of walking in patients with moderate to severe chronic obstructive pulmonary disease. Med Sci Sports Exerc 41: 15401548.

33. Allet L, Armand S, Aminian K, Pataky Z, Golay A, et al. (2010) An exercise intervention to improve diabetic patients' gait in a real-life environment. Gait Posture 32: 185-190.

34. Allet L, Armand S, de Bie RA, Golay A, Monnin D, et al. (2010) The gait and balance of patients with diabetes can be improved: a randomised controlled trial. Diabetologia 53: 458-466.

35. Melai T, Schaper N, ljzerman H, Willems P, Lange T, et al. (Submitted) Strength and gait training increases stride length, stride time and stance phase duration, but does not affect joint moments in diabetic polyneuropathy. A randomized controlled trial.

36. Trombetti A, Hars M, Herrmann FR, Kressig RW, Ferrari S, et al. (2011) Effect of music-based multitask training on gait, balance, and fall risk in elderly people: a randomized controlled trial. Arch Intern Med 171: 525-533.

37. Jehn M, Schmidt-Trucksass A, Meyer A, Schindler C, Tamm M, et al. (2011) Association of daily physical activity volume and intensity with COPD severity. Respir Med 105: 1846-1852.

38. Dolmage TE, Maestro L, Avendano MA, Goldstein RS (1993) The ventilatory response to arm elevation of patients with chronic obstructive pulmonary disease. Chest 104: 1097-1100.

39. Bernard S, LeBlanc P, Whittom F, Carrier G, Jobin J, et al. (1998) Peripheral muscle weakness in patients with chronic obstructive pulmonary disease. Am J Respir Crit Care Med 158: 629-634.

40. Gea JG, Pasto M, Carmona MA, Orozco-Levi M, Palomeque J, et al. (2001) Metabolic characteristics of the deltoid muscle in patients with chronic obstructive pulmonary disease. Eur Respir J 17: 939945.

41. Gosselink R, Troosters T, Decramer M (2000) Distribution of muscle weakness in patients with stable chronic obstructive pulmonary disease. J Cardiopulm Rehabil 20: 353-360.

42. Franssen FM, Wouters EF, Baarends EM, Akkermans MA, Schols AM (2002) Arm mechanical efficiency and arm exercise capacity are relatively preserved in chronic obstructive pulmonary disease. Med Sci Sports Exerc 34: 1570-1576.

43. Jakobsson P, Jorfeldt L, Henriksson J (1995) Metabolic enzyme activity in the quadriceps femoris muscle in patients with severe chronic obstructive pulmonary disease. Am J Respir Crit Care Med 151: $374-377$. 
44. Gosker HR, van Mameren H, van Dijk PJ, Engelen MP, van der Vusse GJ, et al. (2002) Skeletal muscle fibre-type shifting and metabolic profile in patients with chronic obstructive pulmonary disease. Eur Respir J 19: 617-625.

45. Kathiresan G, Jeyaraman SK, Jaganathan J (2010) Effect of upper extremity exercise in people with COPD. Journal of Thoracic Disease 2: 223-236.

46. Ries AL, Bauldoff GS, Carlin BW, Casaburi R, Emery CF, et al. (2007) Pulmonary Rehabilitation: Joint ACCP/AACVPR Evidence-Based Clinical Practice Guidelines. Chest 131: 4S-42S.

47. Hodgkin JE, Balchum OJ, Kass I, Glaser EM, Miller WF, et al. (1975) Chronic obstructive airway diseases. Current concepts in diagnosis and comprehensive care. Jama 232: 1243-1260.

48. Velloso M, Jardim JR (2006) Study of energy expenditure during activities of daily living using and not using body position recommended by energy conservation techniques in patients with COPD. Chest 130: $126-132$.

49. Vorrink SN, Kort HS, Troosters T, Lammers JW (2011) Level of daily physical activity in individuals with COPD compared with healthy controls. Respir Res 12: 33.

50. Bossenbroek L, de Greef MH, Wempe JB, Krijnen WP, Ten Hacken NH (2011) Daily physical activity in patients with chronic obstructive pulmonary disease: a systematic review. Copd 8: 306-319.

51. Casaburi R (2007) Activity monitoring in assessing activities of daily living. Copd 4: 251-255.

52. Morgan M (2008) Life in slow motion: quantifying physical activity in COPD. Thorax 63: 663-664.

53. Benzo R (2009) Activity monitoring in chronic obstructive pulmonary disease. J Cardiopulm Rehabil Prev 29: 341-347.

54. Hecht A, Ma S, Porszasz J, Casaburi R (2009) Methodology for using long-term accelerometry monitoring to describe daily activity patterns in COPD. Copd 6: 121-129.

55. Pitta F, Breyer MK, Hernandes NA, Teixeira D, Sant'Anna TJ, et al. (2009) Comparison of daily physical activity between COPD patients from Central Europe and South America. Respir Med 103: 421-426.

56. Troosters $T$ (2009) Physical activity monitoring: a new outcome facing many challenges, but yielding promising results. Copd 6: 82-83.

57. Zuwallack RL (2009) How do we increase activity and participation in our patients? Semin Respir Crit Care Med 30: 708-712.

58. Mador MJ, Patel AN, Nadler J (2011) Effects of pulmonary rehabilitation on activity levels in patients with chronic obstructive pulmonary disease. J Cardiopulm Rehabil Prev 31: 52-59.

59. Singh S, Morgan MD (2001) Activity monitors can detect brisk walking in patients with chronic obstructive pulmonary disease. J Cardiopulm Rehabil 21: 143-148.

60. Moy ML, Matthess K, Stolzmann K, Reilly J, Garshick E (2009) Free-living physical activity in COPD: assessment with accelerometer and activity checklist. J Rehabil Res Dev 46: 277-286.

61. Watz H, Waschki B, Meyer T, Magnussen H (2009) Physical activity in patients with COPD. Eur Respir J 33: 262-272.

62. Troosters T, Sciurba F, Battaglia S, Langer D, Valluri SR, et al. (2010) Physical inactivity in patients with COPD, a controlled multi-center pilot-study. Respir Med 104: 1005-1011.

63. Pitta F, Troosters T, Probst VS, Langer D, Decramer M, et al. (2008) Are Patients with Copd More Active after Pulmonary Rehabilitation ? Chest 134: 273-280.

64. Hernandes NA, Teixeira Dde C, Probst VS, Brunetto AF, Ramos EM, et al. (2009) Profile of the level of physical activity in the daily lives of patients with COPD in Brazil. J Bras Pneumol 35: 949-956.

65. Preece SJ, Goulermas JY, Kenney LPJ, Howard D, Meijer K, et al. (2009) Activity identification using body-mounted sensors - a review of classification techniques. Physiological Measurement 30: R1R33.

66. Uswatte G, Giuliani C, Winstein C, Zeringue A, Hobbs L, et al. (2006) Validity of accelerometry for monitoring real-world arm activity in patients with subacute stroke: evidence from the extremity constraint-induced therapy evaluation trial. Arch Phys Med Rehabil 87: 1340-1345.

67. Coley B, Jolles BM, Farron A, Bourgeois A, Nussbaumer F, et al. (2007) Outcome evaluation in shoulder surgery using 3D kinematics sensors. Gait Posture 25: 523-532. 
68. Botsis T, Hartvigsen G (2008) Current status and future perspectives in telecare for elderly people suffering from chronic diseases. J Telemed Telecare 14: 195-203.

69. Zijlstra W, Aminian K (2007) Mobility assessment in older people: new possibilities and challenges. Eur J Ageing 4: 3-12.

70. Pitta F, Troosters T, Spruit MA, Probst VS, Decramer M, et al. (2005) Characteristics of physical activities in daily life in chronic obstructive pulmonary disease. Am J Respir Crit Care Med 171: 972977. 


\section{Summary}

Many COPD patients experience problems with activities of daily life (ADLs) that cause loss of functional status, independence and social isolation and thus greatly reduce their quality of life. Therefore insight in problematic ADLs and objective metrics of daily physical activity are of key importance to evaluate the success of treatment programs for COPD patients. This thesis addressed the problematic ADLs COPD patients experience and focused on the assessment of objective metrics of daily physical activity of COPD patients entering pulmonary rehabilitation. Furthermore hardware and software needed to enable measurements of daily physical activity of lower and upper extremities were developed and validated as part of this thesis.

The most prevalent problematic ADLs of COPD patients were identified in chapter 2 . In this study 820 COPD patients entering pulmonary rehabilitation reported 2999 problematic ADLs. Walking was found to be the most prevalent problematic ADL. This activity was problematic for $68 \%$ of the patients. In addition this study determined the perceived performance and satisfaction scores for the identified problematic ADLs. $44 \%$ of patients were not satisfied at all with one or more problematic ADLs. Furthermore the relation between the problematic ADLs and 13 clinical determinants previously reported to affect ADLs in COPD patients was explored. The lack of a strong association between problematic ADLs and clinical determinants emphasizes the need of individualized assessment of these ADLs to allow tailored intervention.

To enable data gathering and data analysis of daily physical activity, the CAM, a single-unit accelerometer, was developed and validated to measure lower extremity activities (chapter 3 ) and upper extremity activities (chapter 5). In chapter 3, ten participants with chronic organ failure wore a CAM (placed on the thigh) for one hour while they were videotaped. The study resulted in an almost perfect agreement between time spend on leg activities and postures according to video and CAM. The validity of the CAM to measure intensity and elevation of arm movements was studied in healthy subjects in chapter 5 . The study results give confidence that the CAM worn just above the elbow can provide an objective, real world index of upper-limb use. The CAM provides accurate data on the type and duration of daily activities of the upper and lower extremities in the home environment.

Detailed analyses of walking, the most prevalent problematic ADL of COPD patients, were performed in chapter 4 . In this study walking patterns were studied using triaxial accelerometers during the 6-min walk test, a test that gained importance in evaluating functional status and has prognostic value in COPD patients. On average, COPD patients walk with a lower intensity, a lower cadence and show a higher vari- 
ability of the accelerometer signal during 6-min walk test in comparison with healthy elderly subjects. The difference in this variability remained even if only subjects of both groups with the same walking speed were compared. These results indicate an altered walking pattern in COPD patients, suggesting that COPD patients experience larger balance disturbances during walking.

Arm activities are required for maintenance of self-care and independent living. In chapter 6 arm ADLs in the home environment of patients with COPD and healthy elderly were characterized. Moreover, relative muscle efforts during arm ADLs in a laboratory setting were compared between COPD patients and healthy subjects. Because arm movement intensity can be influenced by leg activity, leg activity was assessed simultaneously. In the home environment patients with COPD have similar duration of arm ADLs compared to healthy subjects after correction for walking, but perform arm activities at a lower intensity. Also in the laboratory setting, a majority of the arm ADLs were performed at a lower intensity by COPD patients. The taskrelated muscle effort was similar or somewhat higher during several domestic arm ADLs compared to healthy elderly subjects. These results suggest that COPD patients try to avoid very high levels of muscle effort by compensating the intensity of their arm movements.

Summarizing, in this thesis problematic activities in daily life of COPD patients entering pulmonary rehabilitation were identified. Furthermore, valid outcome measures of daily physical activities important to COPD patients were obtained. These outcome measures allow setup of tailored intervention aimed to improve performance of ADLs most important to the patients. Future research has to evaluate the effectiveness of these tailored interventions on the performance of problematic activities in the home environment. 


\section{Samenvatting}

De kwaliteit van leven van veel COPD patiënten (COPD is een chronische vorm van astma) wordt ondermijnd door problemen met activiteiten in het dagelijks leven. COPD patiënten verliezen lichamelijke functionaliteit, de onafhankelijkheid van anderen en raken sociaal geïsoleerd omdat ze bijvoorbeeld slecht ter been zijn. Om deze mensen te kunnen helpen is het van belang om een objectieve maat te hebben van fysieke activiteit, om daarmee vast te stellen of behandeling van deze patiënten succesvol is. In dit proefschrift is onderzoek gedaan naar ten eerste problematische activiteiten in het dagelijks leven van COPD patiënten en ten tweede hoe de fysieke activiteit van COPD patiënten die aan een behandeling beginnen, objectief gemeten kan worden. De benodigde hardware en software voor het doen van metingen van fysieke activiteit in het dagelijks leven zijn daarbij ontwikkeld en gevalideerd.

De meest voorkomende problematische activiteiten in het dagelijks leven van COPD patiënten zijn onderzocht in hoofdstuk 2. Hiervoor zijn 820 COPD patiënten die een revalidatieprogramma voor COPD begonnen ondervraagd. Zij rapporteerden 2999 verschillende problematische activiteiten in hun dagelijks leven. Lopen was hierbij de meeste genoemde activiteit, door $68 \%$ van de patiënten. Naast welke activiteiten problematisch ervaren werden, was de patiënten gevraagd een score te geven voor hun prestatie en tevredenheid per activiteit. $44 \%$ van de patiënten bleek ontevreden over elke door hun genoemde activiteit. Ten tweede was gekeken naar de relatie tussen de problematische activiteiten in het dagelijks leven van patiënten en 13 verschillende klinische eigenschappen die in verband zijn gebracht met achteruitgang van fysieke activiteit. De afwezigheid van duidelijke correlatie tussen deze klinische eigenschappen en hun invloed op COPD patiënten maakt duidelijk dat het noodzakelijk is op individuele basis naar patiënten te kijken, om zo te bepalen wat de beste behandeling per patiënt is.

De CAM, een versnellingsmeter, is ontwikkeld en gevalideerd voor het meten van fysieke activiteit van de benen (hoofdstuk 3) en de armen (hoofdstuk 5). Voor het onderzoek naar de benen waren 10 patiënten met chronisch orgaan falen een uur gefilmd terwijl ze de CAM op hun dijbeen droegen. De CAM en de video bleken een zeer goede overeenkomst te laten zien wat betreft de gespendeerde tijd voor been activiteit en de stand van het been. Het onderzoek voor de armen, uitgevoerd met 10 gezonde mensen, toont aan dat een CAM geplaatst net boven de elleboog objectief de activiteit van de armen kan meten. Hiermee is aangetoond dat de CAM nauwkeurig het type en de duur van dagelijkse activiteiten van zowel armen als benen kan meten. 
Lopen, de meest voorkomende problematische activiteit bij COPD patiënten, is in hoofdstuk 4 in detail bestudeerd. Hiervoor zijn looppatronen tijdens een 6 minuten wandeltest gemeten met versnellingssensoren. Deze 6 minuten wandeltest is een klinisch uitvoerbare test die in de laatste jaren vaker gebruikt wordt voor het evalueren van de functionele status en vooruitzichten voor COPD patiënten.

In dit onderzoek is gevonden dat COPD patiënten met een lagere intensiteit en stapfrequentie lopen dan gezonde proefpersonen van dezelfde leeftijd. Daarnaast bleek dat de versnellingssignalen van COPD patiënten een hogere variabiliteit laten zien vergeleken met de controlegroep. Dit verschil in variabiliteit was ook zichtbaar bij het vergelijken van COPD patiënten en gezonde ouderen die met dezelfde snelheid liepen. Dit laat zien dat het looppatroon van COPD anders is van gezonde ouderen, en ten tweede dat ze een grotere verstoring van hun balans hebben tijdens het lopen.

Activiteiten uitgevoerd met de arm zijn van groot belang voor persoonlijke verzorging en het zelfstandig kunnen wonen. In hoofdstuk 6 is in de leefomgeving van mensen onderzoek gedaan naar activiteiten in het dagelijks leven waar armen voor nodig zijn. Daarnaast is in een klinische omgeving de inspanning van spieren tijdens activiteiten in het dagelijks leven gemeten. In dit onderzoek zijn wederom COPD patiënten en gezonde ouderen vergeleken. Omdat de beweging van armen beïnvloed wordt door bewegingen van de benen, is tegelijkertijd de activiteit van de benen gemeten.

In de leefomgeving gebruiken COPD patiënten hun armen gedurende dezelfde tijd als gezonde deelnemers, maar ze voeren de activiteiten uit met een lagere intensiteit. Ook in de klinische omgeving worden de onderzochte dagelijkse activiteiten door COPD patiënten met een lagere intensiteit uitgevoerd. De inspanning van de specifieke spieren die benodigd waren voor huishoudelijke activiteiten waren juist wat hoger vergelijken met gezonde ouderen. Deze resultaten laten zien dat COPD patiënten in staat zijn om hoge inspanning van spieren te vermijden door activiteiten op een lager intensiteitsniveau uit te voeren. Samenvattend zijn in dit proefschrift problematische activiteiten in het dagelijks leven van COPD patiënten die toetreden tot pulmonaire revalidatie geïdentificeerd. Bovendien zijn activiteiten die belangrijk zijn voor COPD patiënten in het dagelijks leven objectief gemeten met behulp van versnellingssensoren. Dit soort objectieve metingen maken het mogelijk om een op maat gemaakt interventieprogramma op te stellen, rekening houdend met wat voor de patiënt het meest van belang is. Vervolgonderzoek moet aantonen of deze patiënt-specifieke programma's ook daadwerkelijk de uitvoering van de problematische activiteiten in het dagelijks leven van COPD patiënten verbeteren. 


\section{Dankwoord}

Voordat ik echt de laatste punt achter dit proefschrift zet wil ik graag nog een aantal mensen bedanken die ieder op hun eigen wijze een rol hebben gespeeld bij de totstandkoming ervan.

Allereerst wil ik de leden van mijn promotie-team bedanken. Promotoren prof. dr. Emiel Wouters en prof. dr. Annemie Schols, tijdens onze bijeenkomsten heeft jullie interesse en heldere visie mij erg gestimuleerd. Copromotoren dr. Kenneth Meijer en dr. Martijn Spruit, jullie hebben de zware taak op je genomen om mij te laten balanceren op het dunne koord van wetenschappelijke waarde en klinische relevantie. Ik ben blij dat ik van twee walletjes heb mogen eten. Kenneth, met veel humor weet jij de krachten van het zooitje naïeve en eigenwijze studenten en promovendi op de uni te bundelen. Dat dingen niet zo lopen als van te voren bedacht is meer regel dan uitzondering en ik heb veel geleerd van de flexibele en creatieve manier van omgaan met ongeplande lastige situaties. Martijn, nadat ik een lange periode in Maastricht had doorgebracht zorgde jij ervoor dat ik me meteen erg welkom voelde op CIRO en snel op de hoogte was van het reilen en zeilen van de organisatie. Ik ben je ook dankbaar voor je altijd heldere en snelle feedback!

Vervolgens wil ik graag alle deelnemers aan het onderzoek bedanken. Zonder hun medewerking zou het onmogelijk zijn geweest om het onderzoek uit te voeren.

Valeria, ik ben erg dankbaar dat je mij wilde bijstaan in de wereld van de statistiek. Ik heb je betrokkenheid en je kritische kijk op mijn thesis proces erg gewaardeerd. Onze gesprekken gaven me vertrouwen in het maken van goede beslissingen.

Ik wil graag alle collega's van de afdeling bewegingswetenschappen op de universiteit bedanken voor het creëren van een fijne plek! Daardoor kwam ik met veel plezier altijd naar Maastricht. Paranimf Rachel, Herman, Tom, Hans, Paul en Kenneth, onze road trip door Zuid Afrika was fantatisch!

Beste collega's van CIRO, dank jullie wel voor al jullie hulp! Jullie zijn een fantastisch goed op elkaar ingespeelde machine die ik soms aardig in de war heb geschopt. Ik heb erg veel geleerd van jullie manier van werken. Joost en Marianne, dank jullie voor jullie long functie trainingen!

Beste studenten, jullie bijdrage aan deze thesis was onmisbaar! Lang niet ieder project heeft tot een paper geleid, maar het werk verzet aan een andere route of een doodlopende weg heeft voor mij evenveel waarde gehad als het werk dat leidde tot een publicatie te vinden in deze thesis. Mijn plezier in het lesgeven en begeleiden van thesissen was groot. Angel, Aniek, Bjel, Coby, Frank, Katharina, 
Koen, Linda, Loek, Maartje, Malou, Marco, Martijn, Martine, Michelle, Mieke, Nika, Rob, Stijn, Suzanne, Wendy en Yvonne bedankt!

Lieve Chris, pap, mam, familie en vrienden, het schrijven van een proefschrift gaat niet lang niet altijd over rozen en dat hebben jullie gemerkt! Heel veel dank voor jullie soms meer dan welkome afleiding, luisterend oor, advies en steun! 


\section{Curriculum Vitae}

Janneke Annegarn was born on September 16, 1984 in Eindhoven, the Netherlands. She attended secondary school at the Van Maerlant Lyceum in Eindhoven and graduated in 2001. She then studied Physical Therapy at the Fontys Hogeschool in Eindhoven and received her diploma as a physical therapist in 2005. In the same year, she started a 1 year master's program at the Faculty of Health Sciences at Maastricht University. She obtained his Master's degree in Human Movement Sciences in 2006. For her master thesis she studied scaling laws in a computational human skeletal model (Anybody). For this work she stayed at Aalborg University in Denmark. For her Master thesis, she received the Catharina Pijls award (2006) for best thesis of the faculty in 2006.

After working for 1 year as a research assistant at Human Movement Sciences (Maastricht University), Janneke started her PhD project entitled "Daily activity monitoring in patients with COPD" at the same department in September 2007. In September 2009 she received the European Respiratory Society Travel Grant in Vienna, Austria.

Since October 2011 Janneke joined the group of Personal Health Solutions at Philips Research where she works on the topic of fall risk assessment and fall prevention using wearable technology. 



\section{List of publications}

Annegarn J, Spruit MA, Uszko-Lencer NH, Vanbelle S, Savelberg HH, Schols AMWJ, Wouters EF, Meijer K;

Objective physical activity assessment in patients with chronic organ failure: a validation study of a new single-unit activity monitor.

Arch Phys Med Rehabil (2011) 92: 1852-1857 e1851

Hernandes NA, Wouters EF, Meijer K, Annegarn J, Pitta F, Spruit MA;

Reproducibility of 6-minute walking test in patients with COPD.

Eur Respir J (2011) 38: 261-267

Vaes AW, Annegarn J, Meijer K, Cuijpers MW, Franssen FM, Wiechert J, Wouters EF, Spruit MA;

The effects of a 'new' walking aid on exercise performance in patients with COPD: A randomized cross-over trial.

Chest (2012) 141(5):1224-32.

Annegarn J, Meijer K, Passos VL, Stute K, Wiechert J, Savelberg HH, Schols AMWJ, Wouters EF, Spruit MA;

Problematic activities of daily life are weakly associated with clinical characteristics in COPD.

J Am Med Dir Assoc (2012) 13: 284-290 
Annegarn J, Spruit MA, Savelberg HH, Willems PJ, Bool C, Schols AMWJ, Wouters EF, Meijer K;

Differences in walking pattern during 6-min walk test between patients with COPD and healthy subjects.

PLoS One 7: e37329 (2012)

Annegarn J, Spruit MA, Schols AM, Savelberg HH, Murgia A, Wouters EF, Meijer K;

Assessment of intensity and elevation of arm movements during activities of daily life.

Submitted for publication

Annegarn J, Spruit MA, Lima Passos V, Savelberg HH, Schols AMWJ, Wouters EF, Meijer K;

Characteristics of daily arm activities in patients with chronic obstructive pulmonary disease.

Submitted for publication 


\section{List of abbreviations}

\begin{tabular}{|c|c|c|c|}
\hline 6MWD & 6 minute walk distance & ICF & $\begin{array}{l}\text { International Classification of Func- } \\
\text { tioning, disability and health }\end{array}$ \\
\hline 6MWT & 6 Minute Walk Test & km & Kilometer \\
\hline ADL & Activities of Daily Living & $\mathrm{kPa}$ & kilopascal \\
\hline AUC & Area Under the Curve & LTOT & Long-Term Oxygen Therapy \\
\hline BMI & Body Mass Index & $\mathrm{MI}$ & $\begin{array}{l}\text { Mean low pass signal in the longitudi- } \\
\text { nal direction }\end{array}$ \\
\hline bpm & beats per minute & $\min$ & Minute \\
\hline $\mathrm{CCQ}$ & Clinical COPD Questionnaire & MMRC & Modified Medical Research Council \\
\hline $\mathrm{CHF}$ & Chronic Hearth Failure & MRC & Medical Research Counsil \\
\hline COPD & $\begin{array}{l}\text { Chronic Obstructive Pulmonary Dis- } \\
\text { ease }\end{array}$ & MVC & Maximal Voluntary Contraction \\
\hline COPM & $\begin{array}{l}\text { Canadian Occupational Performance } \\
\text { Measure }\end{array}$ & $\mathrm{Nm}$ & Newton meter \\
\hline CV & Coefficient of Variation & OLS & Ordinary Least Squares \\
\hline EMG & ElectroMyoGraphy & $\mathrm{PaCO} 2$ & Partial pressure of carbon dioxide \\
\hline FDR & False Discovery Rate & $\mathrm{PaO} 2$ & Partial pressure of oxygen \\
\hline FEV1 & $\begin{array}{l}\text { Forved Expiratory Volume in one (1) } \\
\text { second }\end{array}$ & PASAQ & $\begin{array}{l}\text { Programmable Ambulant Signal } \\
\text { AcQuisition - system }\end{array}$ \\
\hline FFMI & Fat-Free Mass Index & ROC & Receiver Operator Characteristic \\
\hline FMI & Fat Mass Index & RV & Residual Volume \\
\hline FVC & Forced Vital Capacity & SD & Standard Deviation \\
\hline G & Gravity & sec & Second \\
\hline GLMM & Generalized Linear Mixed Models & SEM & Standardized Error of the Mean \\
\hline GOLD & $\begin{array}{l}\text { Global initiative for chronic Obstruc- } \\
\text { tive Lung Disease }\end{array}$ & sEMG & surface ElectroMyoGraphy \\
\hline $\mathrm{h}$ & hour & SGRQ & $\begin{array}{l}\text { St. George's Respiratory Question- } \\
\text { naire }\end{array}$ \\
\hline HADS & Hospital Anxiety and Depression Scale & SMA & Signal Magnitude Area \\
\hline $\mathrm{HbCO}$ & Carboxihemoglobin & TLC & Total Lung Capacity \\
\hline $\mathrm{Hg}$ & Mercury & & \\
\hline
\end{tabular}

